\title{
MOLTEN-CAUSTIC-LEACHING (GRAVIMELT) \\ SYSTEM INTEGRATION PROJECT
}

QUARTERLY REPORT FOR APRIL THROUGH JUNE, 1989

CONTRACT NUMBER DE-AC22-PC91257

JULY 15, 1989

PREPARED BY APPLIED TECHNOLOGY DIVISION

SPACE AND TECHNOLOGY GROUP

ONE SPACE PARK

REDONDO BEACH, CA 90278

PREPARED FOR

U.S. DEPARTMENT OF ENERGY

PITTSBURGH ENERGY TECHNOLOGY CENTER

P.O. BOX 10940 - MS/922-206

PITTSBURGH, PA 15236

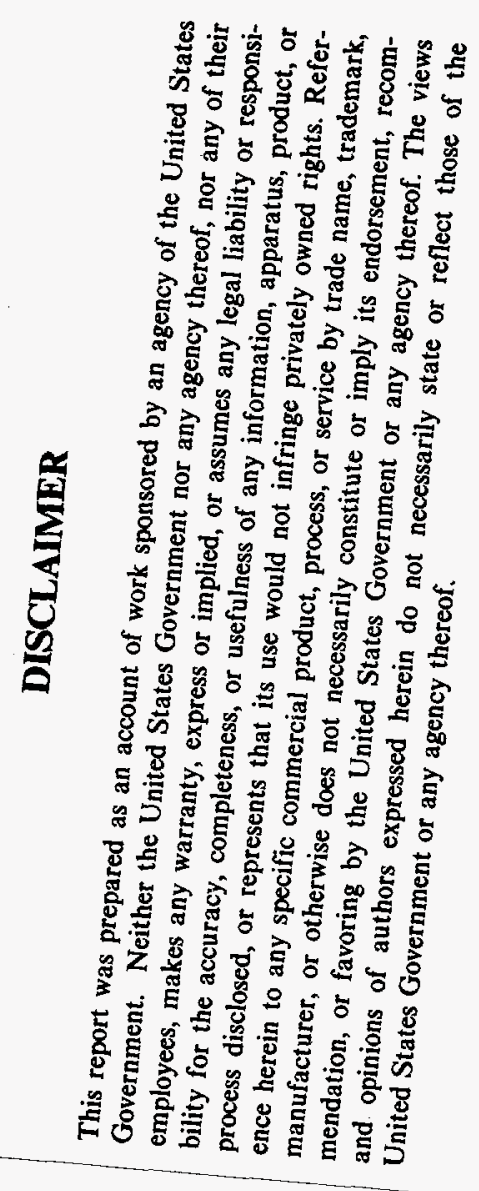




\section{DISCLAIMER}

Portions of this document may be illegible in electronic image products. Images are produced from the best available original document. 


\section{EXECUTIVE SUMMARY}

Operation of the Gravimelt Integrated Test Circuit for desulfurization and demineralization of coal has been completed. A 48-test process matrix was performed over 750 hours of operational time resulting in production of 3,000 pounds of treated coal sultable for further test and evaluation. Optimization testing was performed resulting in product coal containing 0.4 percent sulfur ( 0.6 lbs $\mathrm{SO}_{2} / \mathrm{MMBtu}$ ) and 0.15 percent ash with more than 85 percent organic sulfur removal, 95 percent $\mathrm{SO}_{2}$ reduction from ROM cosi and 91 percent $\mathrm{SO}_{2}$ reduction from precleaned process feed. This report contains all of the product sulfur, ash, volatiles and heat content data obtained to date.

\section{INTRODUCTION AND PROCESS DESCRIPTION}

TRW, under sponsorship of the Department of Energy's Pittsburgh Energy Technology Center (Contract No. DE-AC22-86-PC91257) is evaluating the Gravimelt Process in commercial equipment installed in an Integrated Test circuit. The circuit is located at the TRW San Juan Capistrano test site in California.

The process utilizes a melt of sodium hydroxide or mixtures of sodium and potassium hydroxides at temperatures of $325^{\circ}$ to $415^{\circ} \mathrm{C}$ to remove virtually all of the sulfur and mineral matter from coal. The results of an initial ten weeks of shakedown and operation of the integrated test circuit show that Eastern coals can be treated to meet New Source Performance standards for sulfur oxides control while providing a product with heat content of 14,000 Btu per pound or greater and an ash content as low as a few hundreds of a percent.

The process is seen as a potential replacement for the costly flue gas desulfurization systems contemplated for smaller utilities and industrial boilers. The product coal might also displace oil in some turbine and diesel engines and may have application as an inexpensive preactivated or activated carbon for extraction of pollutants from waste water and can potentially be used to clean-up and concentrate radioactive or toxic waste. Process and plant descriptions are presented in the next 
two sections and a summary of the tests and results of operation of the integrated test circuit are presented in the conclusion of this section.

\subsection{PROCESS DESCRIPTION}

A schematic of the Gravimelt process is shown in Figure 1. Feed coal is first combined with a mixture of dry sodium hydroxide or mixed sodium and potassium hydroxides in a rotary $k i l n$ reactor where the mixture is heated to reaction temperatures of $325^{\circ}$ to $415^{\circ} \mathrm{C}$ causing the caustic to 1) become sorbed in the coal matrix, 2) react and then 3 ) dissolve the coal sulfur and mineral matter.

The reaction mixture exits the rotary kiln in the form of pellets which are next washed with water in a countercurrent separation system of filters and centrifuges. As an example, the use of four weights of wash water per weight of coal results in a coal cake containing up to two parts of wash water per part of coal exiting the last stage of the countercurrent system, and also a 50 percent aqueous reacted caustic solution exiting the first stage of filtration which contains nearly all of the coal-derived silicon, aluminum, carbonate and sulfur content. The coal-derived iron (usually 2-3 percent of the weight of the coal) and a small amount of sodium and/or potassium (0.1-2 percent of the weight of the coal) remains with the coal exiting the countercurrent filtration system.

The coal cake is next washed to remove the residual iron and caustic with, for example, three weights of process water, again in a countercurrent mode. Sulfuric acid is added to the first stage to dissolve the residual alkalt and tron hydroxide: A typical coal cake entering the acid wash filtration train contains two (or as little as one-half) weights of sorbed water while the product coal exiting the train contains one tenth to one weight of sorbed water. Where two weights of wash water enter the acid wash section on the coal cake and the product coal carries one weight of water, the acid extract leaving the first stage of the acid wash section consists of four weights of water containing 250 to 5,000 ppm of mixed sodium and potassium and 5,000 to 10,000 ppm of iron. 


\section{MCL (Gravimelt) System Test Circuit Conditions}

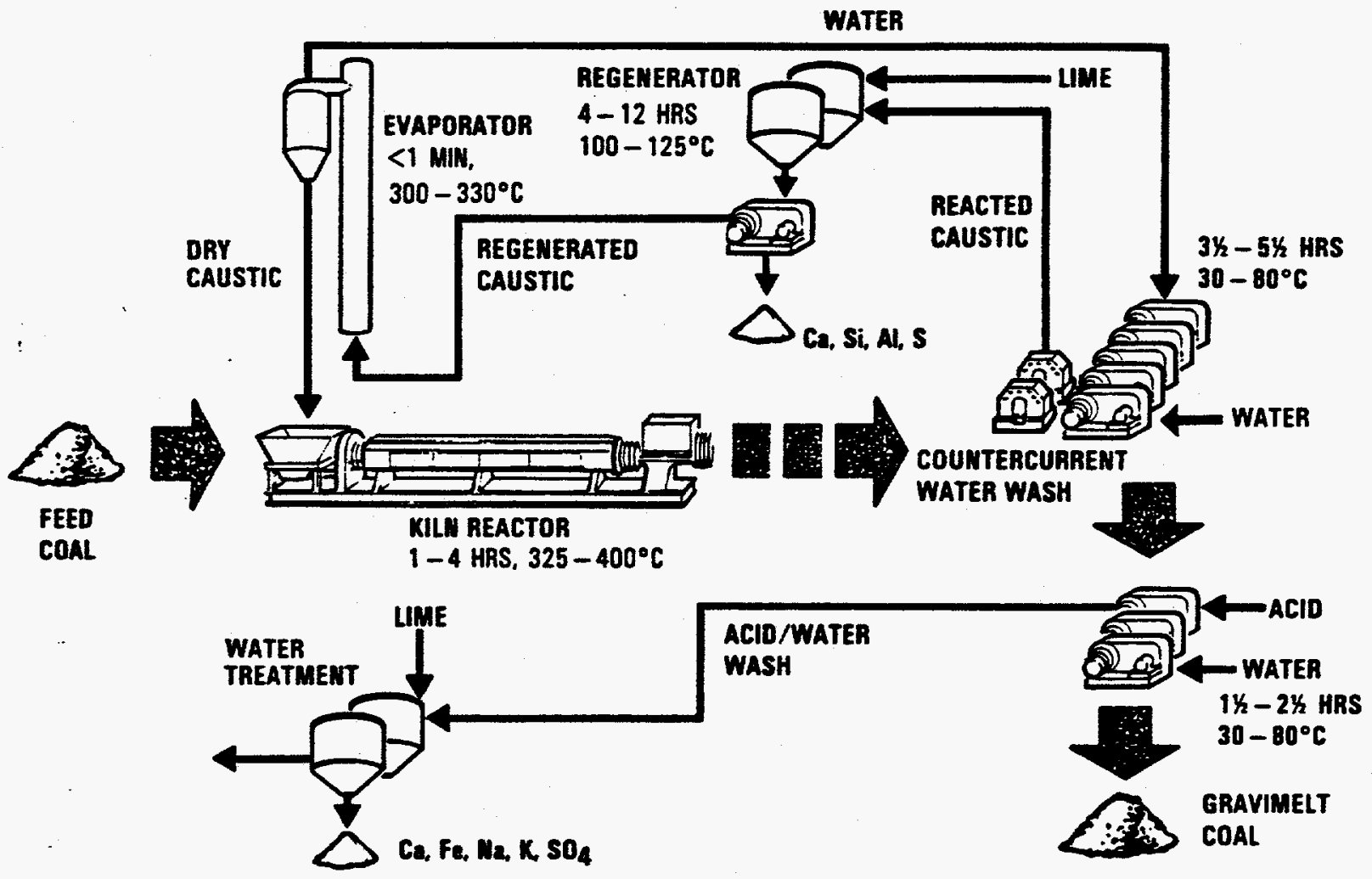

Figure 1. Gravimelt Process Flow Schematic

All of the iron sulfate and a portton of the alkali sulfates in this stream can be removed by a sequence of heating and 11 me treatment to form insoluble minerals. The water can be recycled.

The aqueous caustic is 1 imed at $40^{\circ}$ to $125^{\circ} \mathrm{C}$ to remove the coal derived minerals and carbonate, and the purifled liquid is sent to a caustic evaporator where two weights of wash water are recovered for recycle to the first wash train while producing anhydrous caustic for reuse in extraction of coal. 


\subsection{INTEGRATED TEST CIRCUIT DESCRIPTION}

The test circuit consists of six unit operations (see Figure 1) which together provide a continuous system for leaching coal and regenerating the caustic reactant and wash water. These units are a) a kiln for reacting molten caustic with coal, b) a seven stage water washing section for recovering caustic from the coal, c) a three stage acid washing section for removing the last traces of metals and alkall and providing an ultra pure coal product, d) a water treatment section to provide either dischargeable or recyclable water, e) a regeneration section to provide purified aqueous caustic and $f$ ) an evaporator section to provide molten caustic for recycle to the $k i l n$ reactor.

The integrated test circuit facility contains more than 160 pieces of equipment including filters, centrifuges, tanks, reactors, feeders and the $k i l n$ and rising film evaporator. It occuples 3,700 square feet and is fitted with more than 6,000 feet of piping, 425 vaives, 80 instruments and controls as well as a control room with computer control and data acquisition and reduction system.

\subsection{RESULTS OF SHAKEDOWN AND OPERATION}

The Integrated Test circuit was operated on an around-the-clock basis for ten operating weeks resulting in 750 hours of matrix testing over the period of time from January through May of 1989. More than 4,000 separate analyses were obtained on process streans such as product coal, slurries and filtrates. Varfous solids and additional analyses are yet to be performed. These data are to be assessed in detail. An initial summary is presented below.

1) A 48 test main paraneter operational matrix has been performed with vartation in $\mathrm{k} i \mathrm{in}$ temperature, $325-415^{\circ} \mathrm{C}$; residence time, 1-4 hours; sodium hydroxide or mixtures with potassium hydroxide; ratio of caustic to coal, 1:1 to 3:1; coal particle stze 14 mesh or 6 mesh $\times 0$; and two coals (Pittsburgh No. 8 and Kentucky No. 9) - with the Pittsburgh No. 8 seam being emphasized in this test series. 
2) As a part of the above matrlx, the plant was operated to produce Gravimelt coal with virtually no loss of heat content, no loss of volatiles, near-zero carbonate formation, 0.5 to 2 weights of moisture per weight of coal in the caustic wash section, 0.1 to 1 weights of moisture to coal in the acid wash section and can utilize sodium hydroxide in place of mixed sodium and potassium hydroxides or 6 mesh $x 0$ coal in place of 14 mesh $\times 0$ coal.

3) Produced 3,000 lbs (dry basis) of Gravimelt coal varying in quality from 0.4 percent sulfur to 2.5 percent sulfur, 0.1 percent ash to 2.8 percent ash, $14,250 \mathrm{Btu} / \mathrm{lb}$ to $12,600 \mathrm{Btu} / \mathrm{ib}$ and volatiles from 40 percent to 21 percent.

4) Organic sulfur removal was at least 30 percent at the lowest temperatures utilized, where zero loss of volatiles and heat content was observed and maximized at 85 percent removal where there was minimal heat content loss.

5) Both Pittsburgh No. 8 and Kentucky No. 9 coals were reduced to NSPS compliance levels.

6) Optimization testing was performed on the Pittsburgh No. 8 coal with sodium hydroxide resulting in product coal containing 0.4 percent sulfur (.06 ibs $\mathrm{SO}_{2} / \mathrm{mmBtu}$ ) and 0.15 percent ash with more than 85 percent organic sulfur removal.

7) Best overall coal products (P1ttsburgh No. 8) to date were prepared with $\mathrm{NaOH}$ at 0.40 to 0.75 percent sulfur; 0.1 to 0.65 percent ash; averaging 14,200 Btu/lb; 25 percent vols; 0.60 lbs $\mathrm{SO}_{2} / 10^{6}$. Btu (95 percent $\mathrm{SO}_{2}$ reduction from ROM and 91 percent from precleaned process feed); and 0.90 lbs $\mathrm{SO}_{2} / 10^{\circ}$ Btu (92 percent $\mathrm{SO}_{2}$ reduction from ROM and 86 percent from precleaned process feed).

All of the product sulfur and ash data obtained in the concluding week of operation (Pittsburgh No. 8 coal) is presented in Figure 2. This was a 4-day week due to the Memorial Day hollday. The run was made under a single set of conditions, varying only in use of $\mathrm{NaOH}$ for the latter part of the week and mixed sodium and potassium hydroxides at the beginning of the week. Sulfur removed averaged 93 percent from ROM and 88 percent from mine cleaned feed. Ash reduction averaged 98 percent from coal feed to the process. For several perlods during the latter part of the week, the ash content was so low as to be essentially immeasurable by the standard ASTM technique. 


\section{OPERATING DATA}

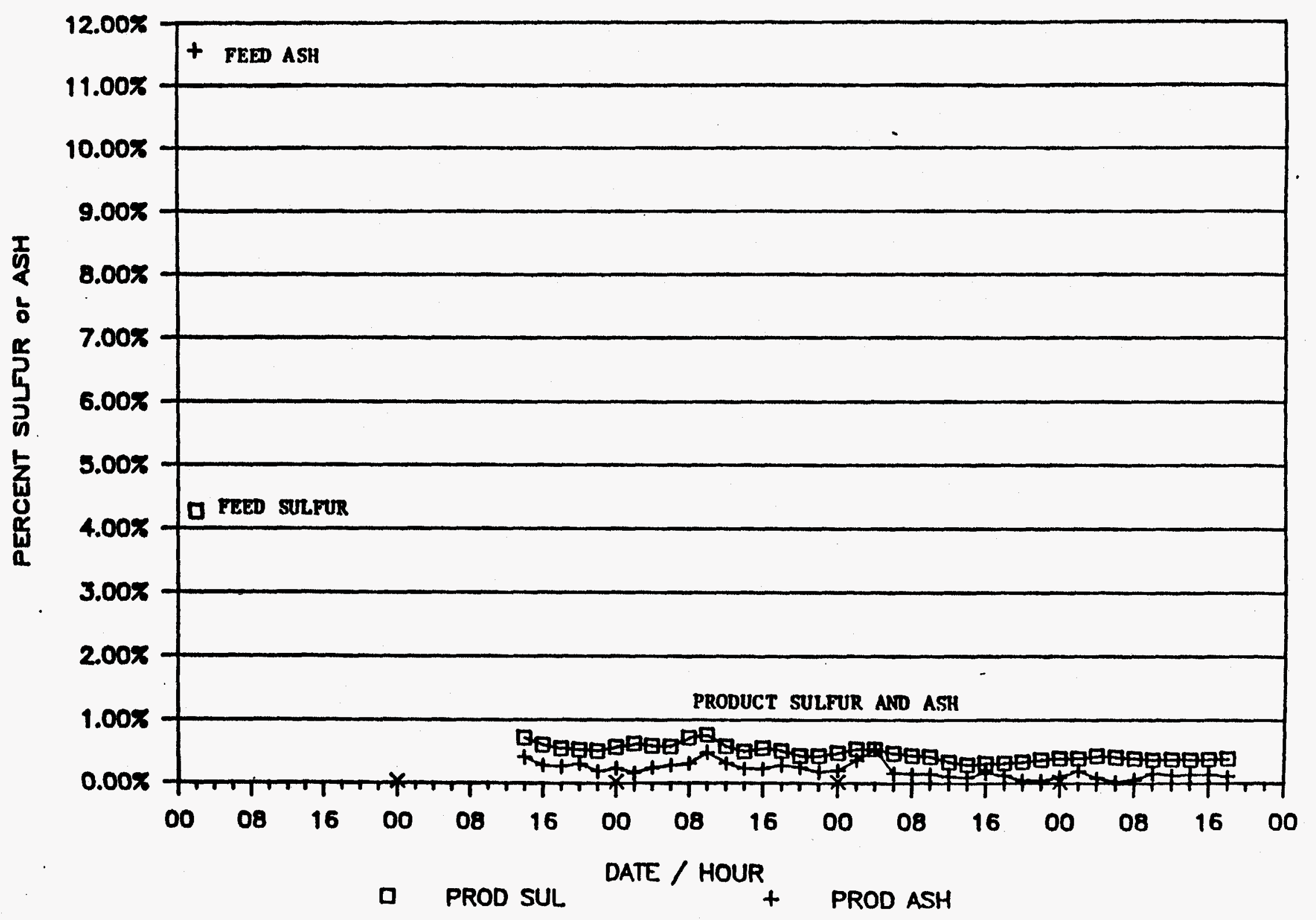

Figure 2. Integrated Teat Circult Product Date 


\section{PROJECT DESCRIPTION}

The objective of this project is to design, construct and operate an integrated test circuit (as shown in Figure 1) for evaluation of the Gravimelt Process and production of desulfurized and demineralized coal samples.

The project consists of elght tasks for planning, design, equipment procurement, construction and shakedown, test planning, operation, data assessment and management.

\section{PROJECT STATUS}

Test circuit operation, product coal samples analyses and regeneration analyses are presented in the three sections to follow. The Integrated Test Circuit Operation Topical Report, which is in preparation, w1ll contain additional data on the unit operations and data assessment. Parameter assessment and modeling of the process based on the data obtained will be performed and presented in the Final Report.

\subsection{TEST CIRCUIT OPERATION SPECIFICATION}

The test matrix performed during the Task 6 operation effort is presented on the following pages on a run-conditions basis. 
PIT 8

KOH/MaOK=1

Caustic/Coal=1

\begin{tabular}{|c|c|c|c|c|c|c|}
\hline in & iA & $3 A 1$ & $3 A 2$ & $3 A 3$ & 6E] & 4 \\
\hline Teap, C & 390 & 427 & 427 & 427 & 427 & 427 \\
\hline es Tice, to & $2-2.2$ & $2-2.8$ & $2-3.6$ & 2.1 & $2-2.8$ & $2-2.8$ \\
\hline 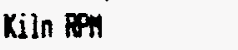 & 2 & 1 & 1 & 1 & 1 & \\
\hline & 30 & 30 & 15 & 30 & 30 & \\
\hline yer sec $F / \sec$ & $20 / 10$ & $20 / 10$ & $20 / 10$ & $17 / 13$ & $20 / 10$ & $20 / 10$ \\
\hline
\end{tabular}

PITT B

KOH/KaOH=1

Cousticicoal $=1.5$

Pun

Teap, C

$\begin{array}{rrrr}18 & 16 & 8 A & 89 \\ 390 & 110 & 127 & 427 \\ 2-2.2 & 2-2.2 & 2-3.0 & 2-3.0 \\ 2 & 2 & 0.8 & 0.8 \\ 30 & 30 & 30 & 30 \\ 20 / 10 & 20 / 10 & 20110 & 20110\end{array}$

thitrogen flow was countereurrent to coal feed in Runs BA and 8B. Also, 6 mesh coal was used in Pun 88.

PITI 8

$\mathrm{KOH} / \mathrm{H} / \mathrm{OH}=1$

Caustic/Coal $=2$

Bun

Teap, $C$

$\begin{array}{rrrrrrrrrr}10 & 2 A & 2 B & X & 383 & 382 & 381 & 5 A 1 & 70 & 7 E \\ 410 & 390 & 390 & 427 & 432 & 438 & 438 & 413 & 427 & 427 \\ 2-2.2 & 2-3.0 & 2-3.0 & 2-3.0 & 2-3.0 & 2-3.6 & 2-3.0 & 2-3.0 & 2-3.0 & 2-3.0 \\ 2 & 211.5 / 1 & 1 & 1 & 1 & 1 & 1 & 1 & 0.8 & 0.8 \\ 30 & 30 & 30 & 30 & 30 & 15 & 30 & 30 & 30 & 30 \\ 20 / 10 & 20 / 10 & 20 / 10 & 20 / 10 & 17 / 13 & 20 / 10 & 20 / 10 & 20 / 10 & 20 / 10 & 20 / 10\end{array}$

Witrogen flow was countercurrent to coal feed in Auns 70 and $Z \mathbb{E}$. Also, recycled caustic was fed with coal in Aun $7 D$.

PITT 8

KOH/KaOH $=1$

Coustic/Coal $=2.5$

An

Ieap, C

$\begin{array}{rrr}94 & 991 & 98 \\ 127 & 438 & 438 \\ 2-3.0 & 2-3.0 & 2-3.0 \\ 0.8 & 0.8 & 0.8 \\ 30 & 30 & 30 \\ 20110 & 20 / 10 & 20110\end{array}$

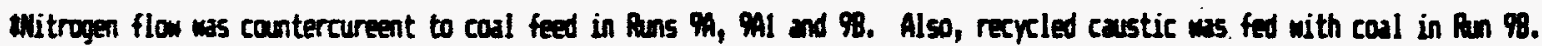


PIT 8

KOH $/ \mathrm{HaOH}=1$

Coustic/Cosl=3

\begin{tabular}{|c|c|c|}
\hline$m_{n}$ & $\mathrm{SCl}$ & 7 \\
\hline Texp, C & 438 & 42 \\
\hline Res Tive, $t r$ & $2-3.0$ & $2-3$. \\
\hline Kiln POH & 1 & 0. \\
\hline Anger Speed, I exx & 30 & \\
\hline Auger sec $F / \sec B$ & $20 / 10$ & $20 / 1$ \\
\hline
\end{tabular}

mitrogen flow mas countercurrent to 6 essh coal feed in fun $\pi$.

PIT 8

KOW/MOH=0

Coustic/Coal=0

Pun 4 A

Teap, C $\quad 410$

Res Time, tor 2-2.8

Kiln RPA 1

Auger Speec, I asx 30

Auger sec F/ser $B$ 20/10

PIIT 8

$\mathrm{KCH} / \mathrm{KaOH}=0$

Coustic/Coal $=1$

hen

Tean, $C$

$6416 \mathrm{Cl}$

fles Tine, her $\quad 2-2.8 \quad 2-2.8$

Kiln PPI 1

Auger Speed, 2 max $30 \quad 30$

Auger sec F/sec $B \quad 20 / 10 \quad 20 / 10$

PIIT 8

KOH/NaOH=O

Coustic/Coal $=1.5$

\begin{tabular}{|c|c|c|c|c|c|}
\hline $\ln 1$ & $\begin{array}{l}681 \\
127\end{array}$ & $\begin{array}{l}601 \\
410\end{array}$ & $\begin{array}{r}x \\
427\end{array}$ & $\begin{array}{r}60 \\
42\end{array}$ & $\begin{array}{r}B E \\
412\end{array}$ \\
\hline Res Tise, to & $2-2.9$ & $2-2.9$ & $2-3.0$ & $2-3.0$ & $2-3.0$ \\
\hline $\ln 804$ & 1 & 1 & 0.8 & 0.8 & 0.8 \\
\hline er Speed, I eax & 30 & 30 & 30 & 30 & 30 \\
\hline er $\sec f / \sec 8$ & $20 / 10$ & 20110 & $20 / 10$ & 20110 & $20 / 10$ \\
\hline
\end{tabular}

witrogen flow was countercurrent to 6 mesh coal ted in Auns $\mathrm{XC}, 80$ and $\mathrm{EC}$. 
PITI 8

KOH $/ \mathrm{NaOH}=0$

Coustic/coal $=2$

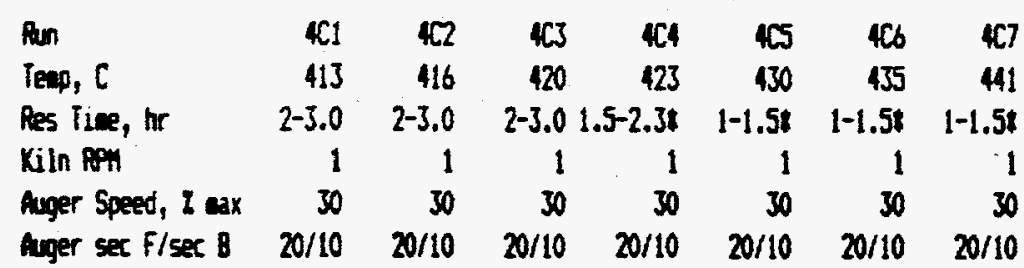

1 Residence time based on heated zones only.

PIT 8

$\mathrm{KOH} / \mathrm{KaOH}=0$

Coustic/Coal $=2$

\begin{tabular}{|c|c|c|c|c|c|c|}
\hline in & 581 & 582 & 583 & 584 & 585 & F \\
\hline Teap, C & 413 & 420 & 423 & 130 & 127 & 412 \\
\hline hes lime, hr & $2-3.0$ & $2-3.01$ & $5-2.38$ & $1-1.5 t$ & $2-3.0$ & $2-3.0$ \\
\hline Kiln PPI & 1 & 1 & 1 & 1 & 1 & 0.8 \\
\hline leed, 2 & 30 & 30 & 30 & 30 & 30 & \\
\hline$F /$ set & $20 / 10$ & $20 / 10$ & $20 / 10$ & $20 / 10$ & 20110 & $20 / 10$ \\
\hline
\end{tabular}

I hesidence tive based on heated zones only.

Nitrogen flow was countercurrent to coal feed in Run \&F. .

PIIT 8

$\mathrm{KBH} / \mathrm{K}: \mathrm{OH}=0$

Custic/Coal=2.5

$\begin{array}{lr}\text { Run } & x \\ \text { Teap, C } & 438 \\ \text { Res Iine, hr } & 2-3.0 \\ \text { Kiln PPH } & 0.8 \\ \text { Auger Speed, 2 adx } & 30 \\ \text { Auger sec F/sec B } & 20 / 10\end{array}$

Ahitrogen flow was countercurrent to coal feed in Run $X$.

KET 9

Kan/Makil

Coustic $/$ cosl $=2$

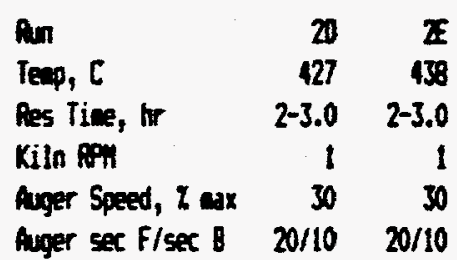


KENT 9

$\mathrm{KOH} / \mathrm{K} 2 \mathrm{OH}=\mathrm{O}$

Curstic/Cos! $=2$

\begin{tabular}{|c|c|c|c|c|c|}
\hline n & $5 C S$ & $5 \mathrm{CA}$ & 501 & 502 & 503 \\
\hline Tex, C & 127 & 127 & 427 & 399 & 371 \\
\hline e, hr & $2-3.0$ & $.5-2.38$ & $1-1.5 t$ & $2-3.0$ & $2-3.0$ \\
\hline 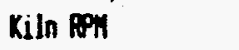 & 1 & 1 & 1 & 1 & \\
\hline & 30 & 30 & 30 & 30 & 30 \\
\hline$c$ c f/sec 8 & $20 / 10$ & $20 / 10$ & $20 / 10$ & $20 / 10$ & $20 / 10$ \\
\hline
\end{tabular}

thesidence tive based on heated zones only.

\section{KEN 9}

KeH/KaOF=1

Custic/Coal=2.5

Aan $7 \mathrm{~A}$

Teep, $C \quad 427$

Res Time, hr 2-3.0

KiIn RPII $\quad 0.8$

Auger Speed, I ax 30

Auger sec F/sec 8 20/10

KENT 9

KOH $/ \mathrm{NaOH}^{2}=1$

Caustie/coal=s

Ran 78

Teap, C 427

Res Time, hro 2-3.0

Kiln 0.8

auger Speed, 2 cax $\quad 30$

auger sec F/sec $8 \quad 20 / 10$ 


\subsection{PRODUCT COAL ANALYSES}

The sulfur and ash content of Integrated Test Circuit product coal is presented on an hour-by-hour basis in the plots to follow. These data are ine-connected and each is followed by a run condition specification table.

Volatiles versus sulfur content and volatiles versus heat content are shown on the next set of plots.

Analyses of intermediate samples taken at the kiln exit are also shown. Approximately four hours should be added to the abscissa of these samples to align with the time of exit of the product coal collected at the final wash station. This is to correct for residence time in the water and acid wash sections.

As a point of reference it should be noted that the feed Pittsburgh No. 8 coal analyses are: 4.27 percent total sulfur; 38 percent volatiles; 2.54 percent organic sulfur; 11.57 percent ash; 13,016 Btu/1b; 14,719 MAF Btu/lb; and the feed Kentucky No. 9 analyses are: 3.53 percent total sulfur; 1.85 percent organic sulfur; 9.21 percent ash; 13,259 Btu/1b; and 14,604 MAF Btu/1b.

The final section contains a listing of all product coal together with anaiyses to aid in selection of samples for delivery to the DOE for testing. 
4.2.1 Sulfur and Ash Data for Product Coal as a Function of Coal Exit Time 


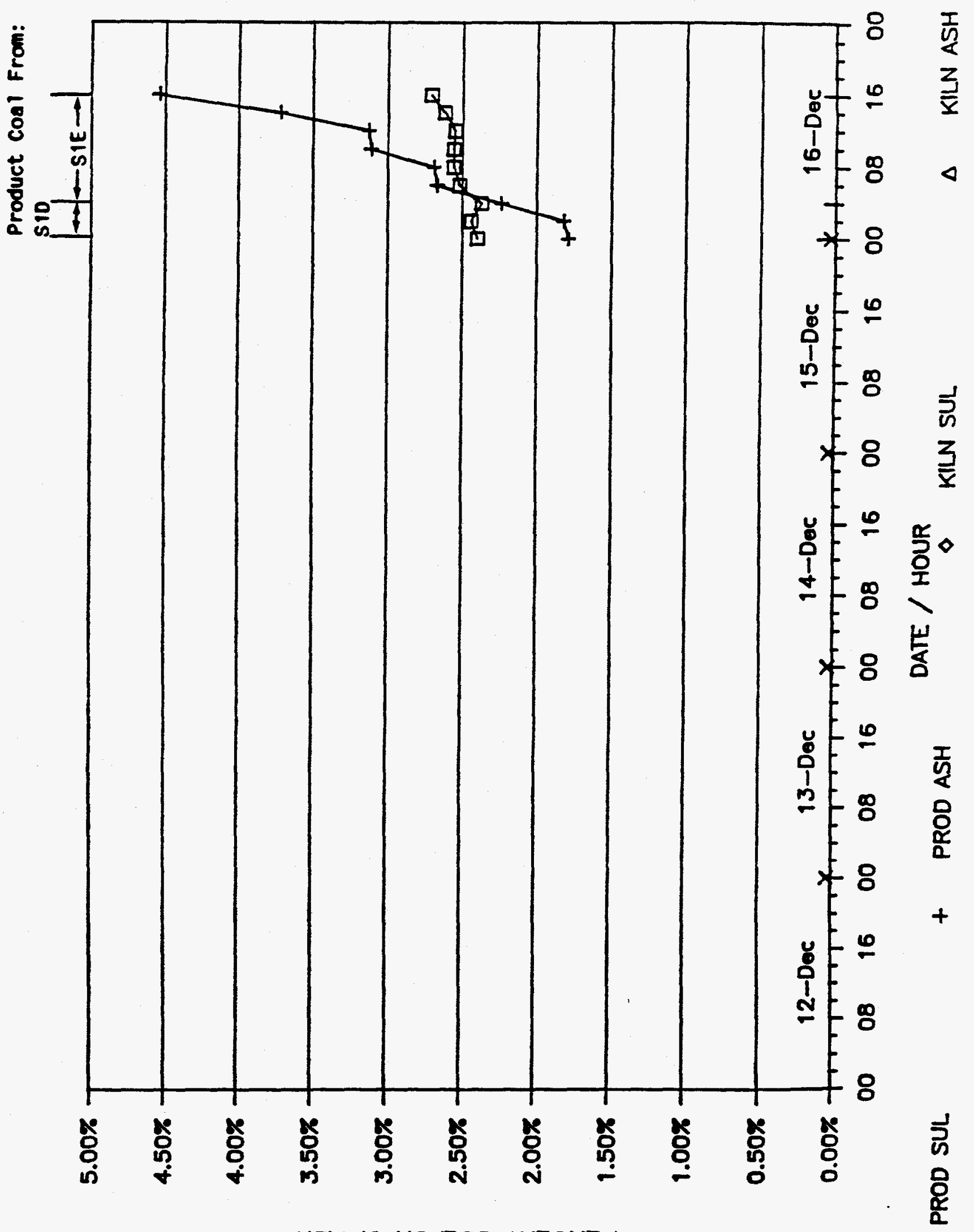

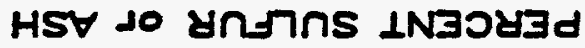




\section{Run Condittion Summary}

Shakedown Run No. 1, 12/12-12/16/88

\begin{tabular}{|c|c|c|c|c|c|c|}
\hline & $\begin{array}{l}\text { Operat in } \\
\text { Span for }\end{array}$ & $\begin{array}{l}n g \text { Time } \\
r k \operatorname{lin} n^{*}\end{array}$ & & & & \\
\hline $\begin{array}{l}\text { Run } \\
\text { No. }\end{array}$ & $\begin{array}{l}\text { Date } \\
\text { From/To }\end{array}$ & $\begin{array}{c}\text { Time } \\
\text { From/To }\end{array}$ & $\begin{array}{l}\text { Coal } \\
\text { Iype }\end{array}$ & $\begin{array}{l}\text { Caustic } \\
\text { Type } \\
\end{array}$ & Caust fc: Coal & $\begin{array}{l}k+1 n \text { Skin } \\
\text { Iemps }{ }^{*}{ }^{* *}\end{array}$ \\
\hline s1A & $\begin{array}{l}12 / 12 \\
12 / 13\end{array}$ & $\begin{array}{l}0800- \\
1200\end{array}$ & $\begin{array}{l}\text { Pittsburgh } 18 \\
14 \text { Mesh }\end{array}$ & $\begin{array}{l}\mathrm{NaOH}: \mathrm{KOH} \\
(50: 50)\end{array}$ & $1: 1$ & 380 \\
\hline S1B & $12 / 13$ & $1200-1500$ & $\begin{array}{l}\text { P1ttsburgh } 18 \\
14 \text { Mesh }\end{array}$ & $\begin{array}{l}\mathrm{NaOH}: \mathrm{KOH} \\
(50: 50)\end{array}$ & $1: 1$ & 290 \\
\hline sic & $12 / 13$ & $1700-2100$ & $\begin{array}{l}\text { Ptttsburgh } 18 \\
14 \text { Mesh }\end{array}$ & $\begin{array}{l}\text { NaOH: KOH } \\
(50: 50)\end{array}$ & $1: 1$ & 335 (Zone 1),-315 (Zones 2-4) \\
\hline $\mathbf{S 1 0}$ & $\begin{array}{l}12 / 13 \\
12 / 15\end{array}$ & $\begin{array}{l}2200- \\
2100\end{array}$ & $\begin{array}{l}\text { P1ttsburgh } \$ 8 \\
14 \text { Mesh }\end{array}$ & $\begin{array}{l}\text { NaOH:KOH } \\
(50: 50)\end{array}$ & $1: 1$ & 350 (Zone 1$),-330($ Zones 2-4) \\
\hline SIE & $\begin{array}{l}12 / 15 \\
12 / 16\end{array}$ & $\begin{array}{l}2200- \\
0800\end{array}$ & $\begin{array}{l}\text { P1ttsburgh } 18 \\
14 \text { Mesh }\end{array}$ & $\begin{array}{l}\text { NaOH: KOH } \\
(50: 50)\end{array}$ & $1: 1$ & $360--100$ e 12/16, 0800 \\
\hline
\end{tabular}

* It has been calculated that it takes -8 hours for the coal to get thru the $k+1 n$ and wash trains. Therefore, when relating product coal collected to $k i l n$ conditions this 8 hour lag time must be taken into account.

* Established in later runs that $k 1$ In internal equilibrlum process temperature $-20-25^{\circ} \mathrm{C}$ lower than external kiln wall skin temperature. 


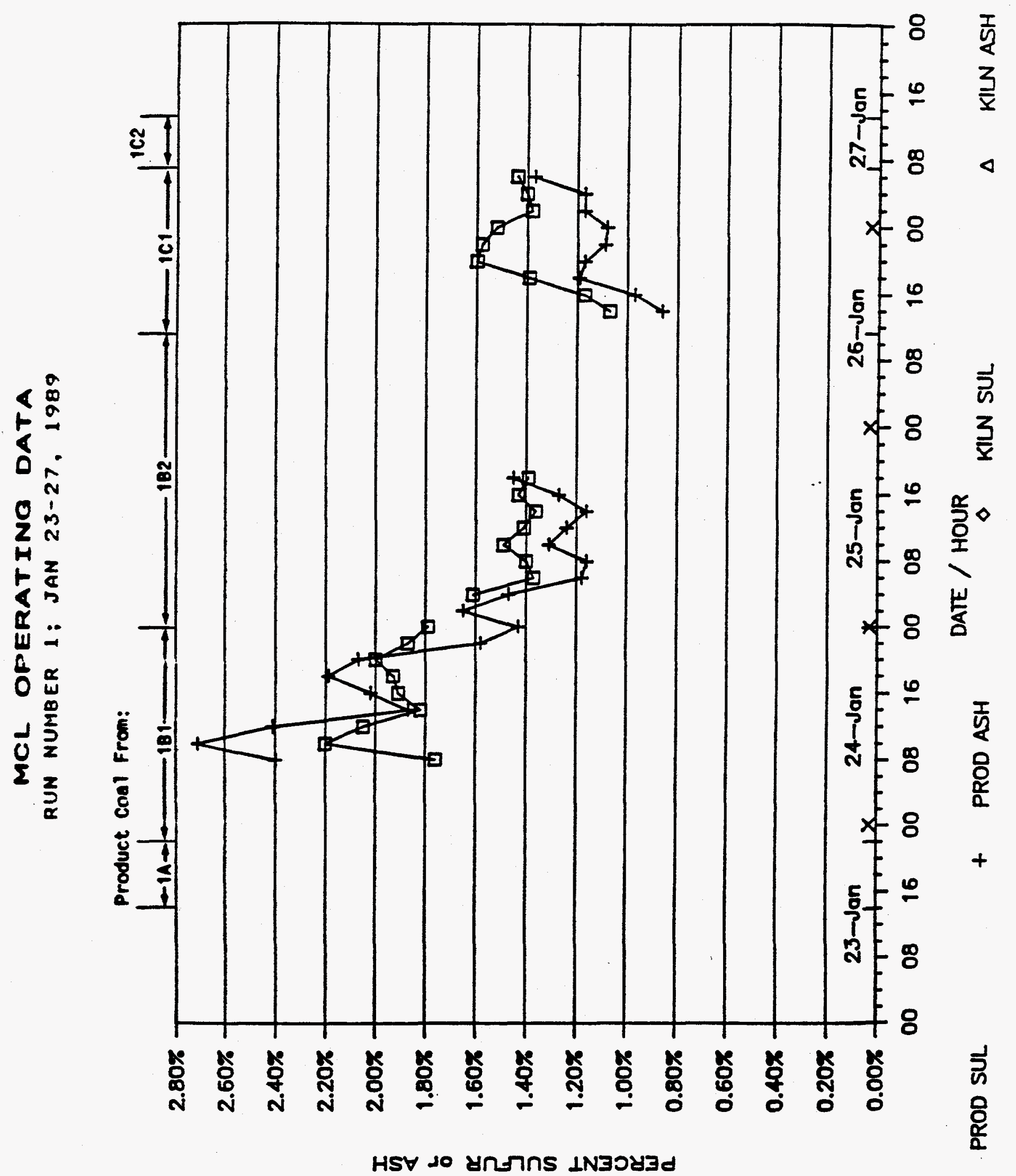

HS 


\section{Run Conditton Summary.}

Operat ing Run No. 1, 1/23-1/27/89

\begin{tabular}{|c|c|c|c|c|c|c|}
\hline \multirow[b]{2}{*}{$\begin{array}{l}\text { Run } \\
\text { No. }\end{array}$} & \multicolumn{2}{|c|}{$\begin{array}{l}\text { Operating Time } \\
\text { Span for } k+1 n^{*}\end{array}$} & \multirow[b]{2}{*}{$\begin{array}{l}\text { Coal } \\
\text { Iype } \\
\end{array}$} & \multirow[b]{2}{*}{$\begin{array}{l}\text { Caust ic } \\
\text { Type }\end{array}$} & \multirow[b]{2}{*}{ Caust 1c:Coal } & \multirow[b]{2}{*}{ KtIn Skin Temps ${ }^{*}$} \\
\hline & $\begin{array}{l}\text { Date } \\
\text { From/To }\end{array}$ & $\begin{array}{l}\text { Time } \\
\text { From/to }\end{array}$ & & & & \\
\hline $1 A$ & $1 / 23$ & $0400-1430$ & $\begin{array}{l}\text { Pittsburgh } \$ 8 \\
14 \text { Mesh }\end{array}$ & $\begin{array}{l}\text { NaOH:KOH } \\
(50: 50)\end{array}$ & $1: 1$ & 390 \\
\hline 1B1 & $\begin{array}{l}1 / 23 \\
1 / 24\end{array}$ & $\begin{array}{l}1430- \\
1630\end{array}$ & $\begin{array}{l}\text { P1ttsburgh } 18 \\
14 \text { Mesh }\end{array}$ & $\begin{array}{l}\text { NaOH: KOH } \\
(50: 50)\end{array}$ & $1.5: 1$ & 390 \\
\hline 182 & $\begin{array}{l}1 / 24 \\
1 / 26\end{array}$ & $\begin{array}{l}1630- \\
0300\end{array}$ & $\begin{array}{l}\text { Pittsburgh } 18 \\
14 \text { Mesh }\end{array}$ & $\begin{array}{l}\text { NaOH:KOH } \\
(50: 50)\end{array}$ & $1.5: 1$ & 427 \\
\hline $1 C 1$ & $1 / 26$ & $0300-2330$ & $\begin{array}{l}\text { Pittsburgh } 18 \\
14 \text { Mesh }\end{array}$ & $\begin{array}{l}\text { MaOH:KOH } \\
(50: 50)\end{array}$ & $2: 1$ & 427 \\
\hline $1 C 2$ & $\begin{array}{l}1 / 26 \\
1 / 27\end{array}$ & $\begin{array}{l}2330- \\
1400\end{array}$ & $\begin{array}{l}\text { P1ttsburgh } 18 \\
14 \text { Mesh }\end{array}$ & $\begin{array}{l}\text { NaOH:KOH } \\
(50: 50)\end{array}$ & $2: 1$ & 438 \\
\hline
\end{tabular}

* It has been calculated that it takes -8 hours for the coal to get thru the k11n and wash trains. Therefore, when relating product coal collected to $k 1$ in conditions this 8 hour lag t ime must be taken into account.

* Established in later runs that $k 1$ In internal equilibrium process temperature $-20-25^{\circ} \mathrm{C} 10$ wer than external kiln wall skin temperature. 
MCL OPERATING DATA

RUN NUMBER 2: FEB 06-10, 1989

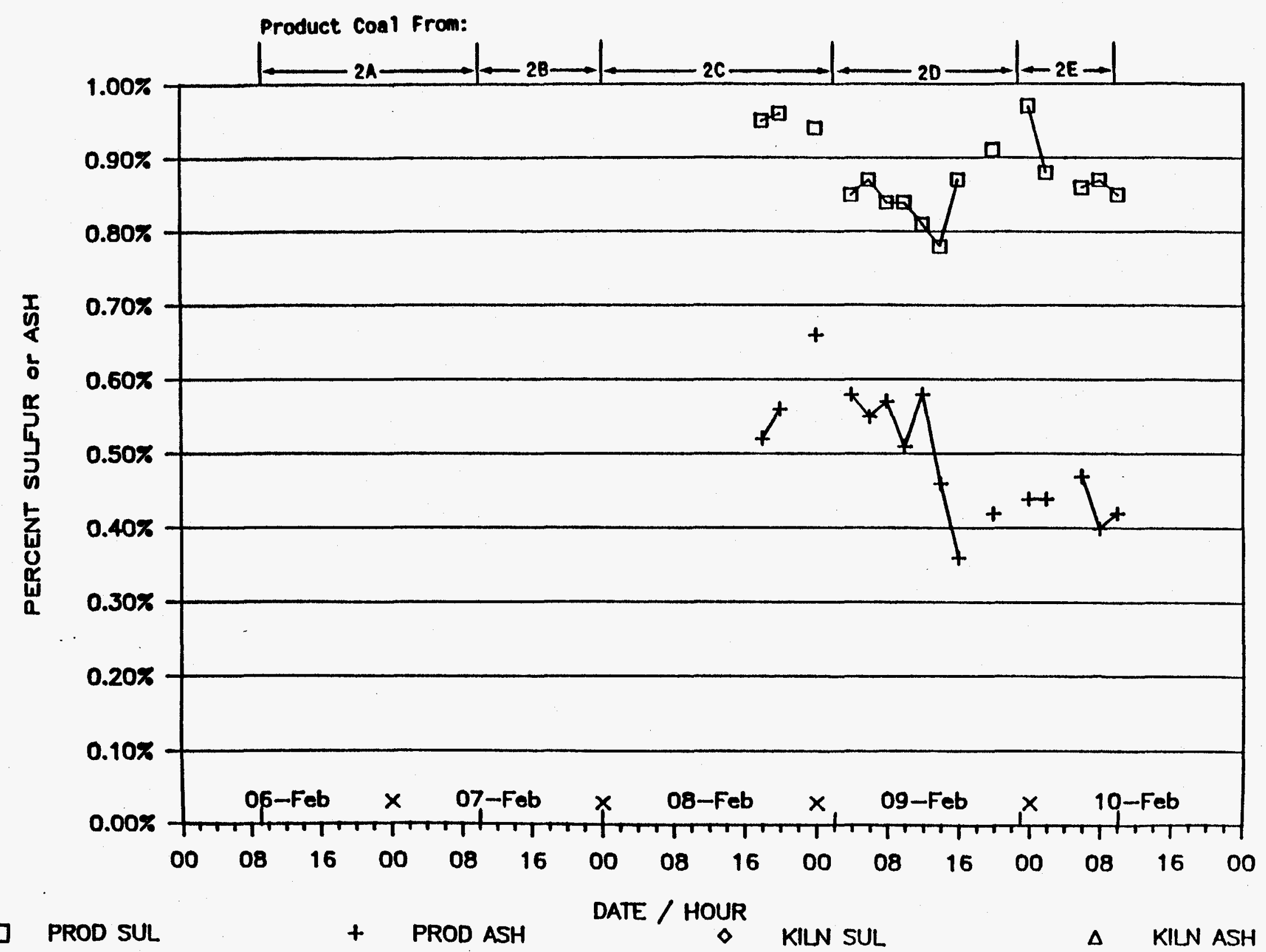




\section{Run Cond1t ton Summary}

Operating Run No. 2, 2/06-2/10/89

\begin{tabular}{|c|c|c|c|c|c|c|}
\hline \multirow[b]{2}{*}{$\begin{array}{l}\text { Run } \\
\text { No. }\end{array}$} & \multicolumn{2}{|c|}{$\begin{array}{l}\text { Operating TIme } \\
\text { Span for } K+1 n^{*}\end{array}$} & \multirow[b]{2}{*}{$\begin{array}{l}\text { Coal } \\
\text { Iype }\end{array}$} & \multirow[b]{2}{*}{$\begin{array}{l}\text { Caust 1c } \\
\text { Iype } \\
\end{array}$} & \multirow[b]{2}{*}{ Causttc:Coal } & \multirow[b]{2}{*}{$\begin{array}{l}\text { Kiln Skin } \\
\text { Iemps }{ }^{\circ} \mathrm{C}^{*}\end{array}$} \\
\hline & $\begin{array}{l}\text { Date } \\
\text { From/To }\end{array}$ & $\begin{array}{l}\text { TIme } \\
\text { From/To }\end{array}$ & & & & \\
\hline $2 A$ & $\begin{array}{l}2 / 06 \\
2 / 07\end{array}$ & $\begin{array}{l}0145- \\
0225\end{array}$ & $\begin{array}{l}\text { P1ttsburgh } 18 \\
14 \text { Mesh }\end{array}$ & $\begin{array}{l}\mathrm{NaOH}: \mathrm{KOH} \\
(50: 50)\end{array}$ & $2: 1$ & 390 \\
\hline $2 B$ & $2 / 07$ & 0225-1535 & $\begin{array}{l}\text { P1ttsburgh } 18 \\
14 \text { Mesh }\end{array}$ & $\begin{array}{l}\text { MaOH:KOH } \\
(50: 50)\end{array}$ & $2: 1$ & 390 \\
\hline $2 C$ & $\begin{array}{l}2 / 07 \\
2 / 08\end{array}$ & $\begin{array}{l}1535- \\
1815\end{array}$ & $\begin{array}{l}\text { P1ttsburgh } 18 \\
14 \text { Mesh }\end{array}$ & $\begin{array}{l}\text { NaOH: KOH } \\
(50: 50)\end{array}$ & $2: 1$ & 427 \\
\hline 20 & $\begin{array}{l}2 / 08 \\
2 / 09\end{array}$ & $\begin{array}{l}1815- \\
1500\end{array}$ & $\begin{array}{l}\text { Kentucky } 19 \\
14 \text { Mesh }\end{array}$ & $\begin{array}{l}\text { NaOH:KOH } \\
(50: 50)\end{array}$ & $2: 1$ & 427 \\
\hline $2 E$ & $\begin{array}{l}2 / 09 \\
2 / 10\end{array}$ & $\begin{array}{l}1500- \\
1300\end{array}$ & $\begin{array}{l}\text { Kentucky } \\
14 \text { Mesh }\end{array}$ & $\begin{array}{l}\text { NaOH: KOH } \\
(50: 50)\end{array}$ & $2: 1$ & 438 \\
\hline
\end{tabular}

* It has been calculated that it takes - 8 hours for the coal to get thru the $k 1$ in and wash trains. Therefore, when relating product coal collected to $k 1$ ln conditions this 8 hour lag time must be taken into account.

* Established in later runs that $k+1 n$ Internal equilibrium process temperature -20-25\% 10 wer than external kiln wall skin temperature. 
MCL OPERATING DATA

- RUN NUMBER 3: FEB 20-24, 1989

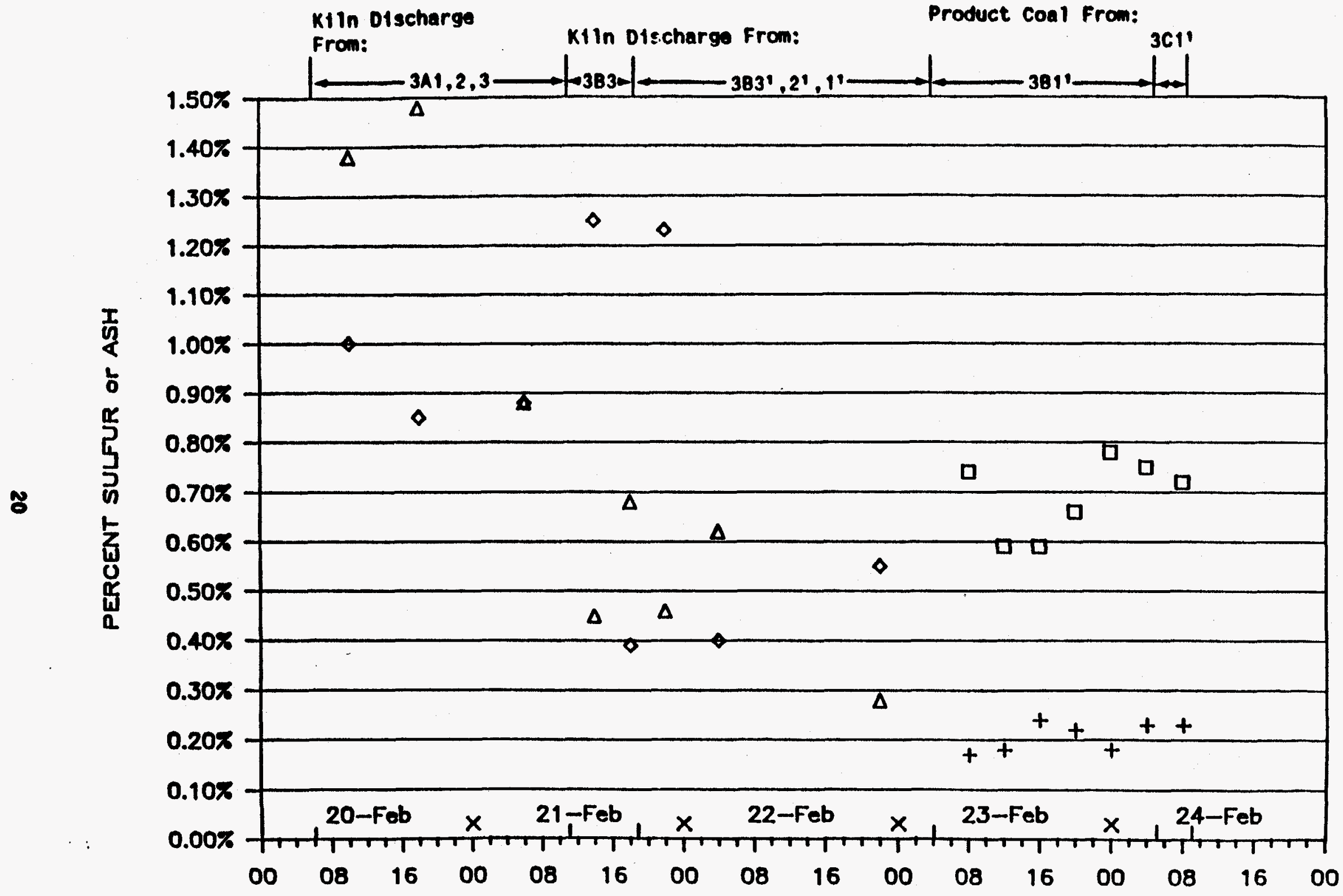

口 PROD SUL

$+\quad$ PROD ASH

DATE / HOUR

$\diamond \quad$ KILN SUL

$\triangle \quad$ KILN ASH 
Operat Ing Run No. 3, 2/20-2/24/89

\begin{tabular}{|c|c|c|c|c|c|c|}
\hline \multirow[b]{2}{*}{$\begin{array}{l}\text { Run } \\
\text { No. }\end{array}$} & \multicolumn{2}{|c|}{$\begin{array}{l}\text { Operating Time } \\
\text { Span for K1ln }\end{array}$} & \multirow[b]{2}{*}{$\begin{array}{l}\text { Coal } \\
\text { Iype }\end{array}$} & \multirow[b]{2}{*}{$\begin{array}{l}\text { Causttc } \\
\text { Type }\end{array}$} & \multirow[b]{2}{*}{ Caust 1c:Coal } & \multirow[b]{2}{*}{$\begin{array}{l}\text { Klin Skin } \\
\text { Temps }{ }^{*} C^{* * \ldots}\end{array}$} \\
\hline & $\begin{array}{l}\text { Date } \\
\text { From/To }\end{array}$ & $\begin{array}{l}\text { Time } \\
\text { From/To }\end{array}$ & & & & \\
\hline $\begin{array}{l}3 A 1, \\
A 2, A 3 *=\end{array}$ & $\begin{array}{l}2 / 20 \\
2 / 21\end{array}$ & $\begin{array}{l}0130- \\
0650\end{array}$ & $\begin{array}{l}\text { P1ttsburgh } \$ 8 \\
14 \text { Mesh }\end{array}$ & $\begin{array}{l}\mathrm{NaOH}: \mathrm{KOH} \\
(50: 50)\end{array}$ & $1: 1$ & 427 \\
\hline 3B3 & $2 / 21$ & $0720-1520$ & $\begin{array}{l}\text { P1ttsburgh } 18 \\
14 \text { Mesh }\end{array}$ & $\begin{array}{l}\mathrm{NaOH}: \mathrm{KOH} \\
(50: 50)\end{array}$ & $2: 1$ & 427 \\
\hline $\begin{array}{l}38^{1}, 82^{1} \\
81^{1}: 4\end{array}$ & $\begin{array}{l}2 / 21 \\
2 / 24\end{array}$ & $\begin{array}{l}1520- \\
0000\end{array}$ & $\begin{array}{l}\text { P1ttsburgh } 18 \\
14 \text { Mesh }\end{array}$ & $\begin{array}{l}\text { NaOH: KOH } \\
(50: 50)\end{array}$ & $2: 1$ & 438 \\
\hline $3 C 11$ & $2 / 24$ & $0100-0830$ & $\begin{array}{l}\text { P1ttsburgh } 8 \\
14 \text { Mesh }\end{array}$ & $\begin{array}{l}\mathrm{NaOH}: \mathrm{KOH} \\
(50: 50)\end{array}$ & $3: 1$ & 438 \\
\hline
\end{tabular}

* It has been calculated that it takes -8 hours for the coal to get thru the ktin and wash trains. Therefore, when relating product coal collected to $k i l n$ conditions this 8 hour lag time must be taken into account.

** Tests A1-A3 differ in kiln internal auger setting.

*** Tests $81^{1-831}$ differ In kiln internal auger setting.

**** Established in later runs that $k 1$ in internal equilibrlum process temperature $-20-25^{\circ} \mathrm{C}$ lower than external ktln wall skin temperature. 


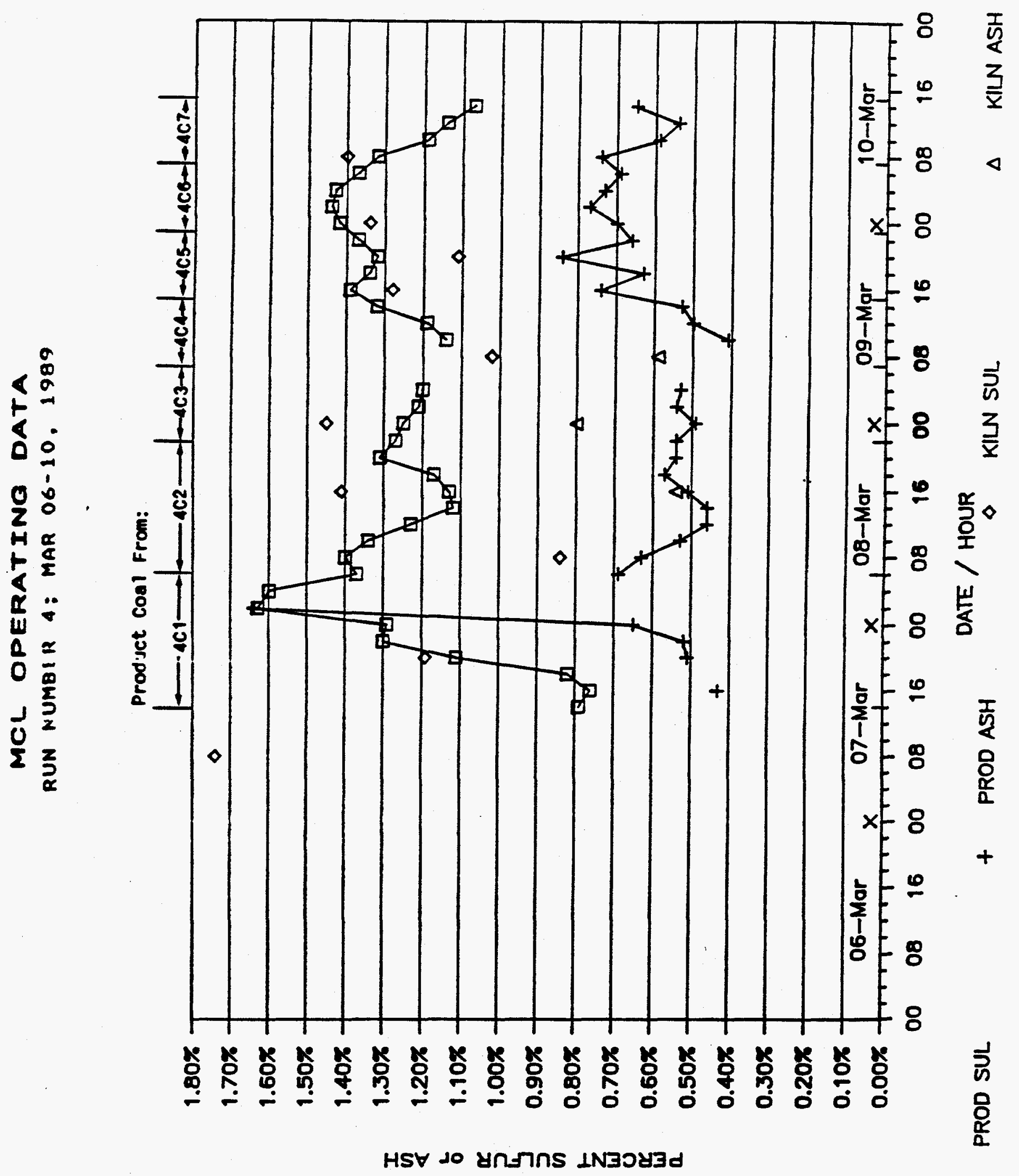

HS 


\section{Run Condition Summary}

Operating Run No. $4,3 / 06-3 / 10 / 89$

\begin{tabular}{|c|c|c|c|c|c|c|c|c|c|}
\hline \multirow[b]{2}{*}{$\begin{array}{l}\text { Run } \\
\text { No. }\end{array}$} & \multicolumn{2}{|c|}{$\begin{array}{l}\text { Operat ing TIme } \\
\text { Span for } K+1 n^{*}\end{array}$} & \multirow[b]{2}{*}{$\begin{array}{l}\text { Coal } \\
\text { Type }\end{array}$} & \multirow[b]{2}{*}{$\begin{array}{l}\text { Caust tc } \\
\text { Type } \\
\end{array}$} & \multirow[b]{2}{*}{ Caust 1c:Coal } & \multirow{2}{*}{\multicolumn{2}{|c|}{ Kilin Skin Temps:* }} & \multirow[b]{2}{*}{ Zone } & \multirow[b]{2}{*}{ Zone 4} \\
\hline & $\begin{array}{l}\text { Date } \\
\text { From/To }\end{array}$ & $\begin{array}{c}\text { Trme } \\
\text { From/To }\end{array}$ & & & & & & & \\
\hline $\mathbb{A A}$ & $3 / 06$ & $0130-1020$ & $\begin{array}{l}\text { Pittsburgh } 18 \\
14 \text { Mesh }\end{array}$ & None & Coal only & 410 & 410 & 410 & 410 \\
\hline 4C1 & $\begin{array}{l}3 / 06 \\
3 / 07\end{array}$ & $\begin{array}{l}1630- \\
2200\end{array}$ & $\begin{array}{l}\text { Pittsburgh } \\
14 \text { Mesh }\end{array}$ & $\mathrm{NaOH}$ only & $2: 1$ & 421 & 410 & 410 & 410 \\
\hline 4C2 & $\begin{array}{l}3 / 07 \\
3 / 08\end{array}$ & $\begin{array}{l}2200- \\
1430\end{array}$ & $\begin{array}{l}\text { P1ttsburgh } 8 \\
14 \text { Mesh }\end{array}$ & $\mathrm{NaOH}$ only & $2: 1$ & 432 & 410 & 410 & 410 \\
\hline 403 & $3 / 08$ & $1540-2300$ & $\begin{array}{l}\text { Pittsburgh } \\
14 \text { Mesh }\end{array}$ & $\mathrm{NaOH}$ only & $2: 1$ & 449 & 410 & 410 & 410 \\
\hline $4 C 4$ & $3 / 09$ & 0100-0700 & $\begin{array}{l}\text { P1ttsburgh } 8 \\
14 \text { Mesh }\end{array}$ & $\mathrm{NaOH}$ only & $2: 1$ & $\begin{array}{l}\text { Heater } \\
\text { Off }\end{array}$ & 449 & 410 & 410 \\
\hline 405 & $3 / 09$ & $0810-1500$ & $\begin{array}{l}\text { Pittsburgh } 8 \\
14 \text { Mesh }\end{array}$ & $\mathrm{NaOH}$ only & $2: 1$ & $\begin{array}{l}\text { Heater } \\
\text { Off }\end{array}$ & $\begin{array}{l}\text { Heater } \\
\text { Off }\end{array}$ & 449 & 410 \\
\hline 406 & $3 / 09$ & $1515-2300$ & $\begin{array}{l}\text { P1ttsburgh } 18 \\
14 \text { Mesh }\end{array}$ & $\mathrm{NaOH}$ only & $2: 1$ & $\begin{array}{l}\text { Heater } \\
\text { off }\end{array}$ & $\begin{array}{l}\text { Heater } \\
\text { off }\end{array}$ & 449 & 421 \\
\hline $4 C 7$ & $\begin{array}{l}3 / 09 \\
3 / 10\end{array}$ & $\begin{array}{l}2315- \\
1300\end{array}$ & $\begin{array}{l}\text { P1ttsburgh } 18 \\
14 \text { Mesh }\end{array}$ & $\mathrm{NaOH}$ only & $2: 1$ & $\begin{array}{l}\text { Heater } \\
\text { Off }\end{array}$ & $\begin{array}{l}\text { Heater } \\
\text { off }\end{array}$ & 449 & 432 \\
\hline
\end{tabular}

* It has been calculated that it takes -8 hours for the coal to get thru the $k 1$ ln and wash trains. Therefore, when relating product coal collected to $k$ iln conditions this 8 hour lag t Ime must be taken into account.

* Established in later runs that $k+1 n$ internal equilibrium process temperature $-20-25^{\circ} \mathrm{C} 10$ wer than external $k+l n$ wall skin temperature. 
MCL OPERATING DATA

RUN NUMBER 5: MAR 20-24, 1989

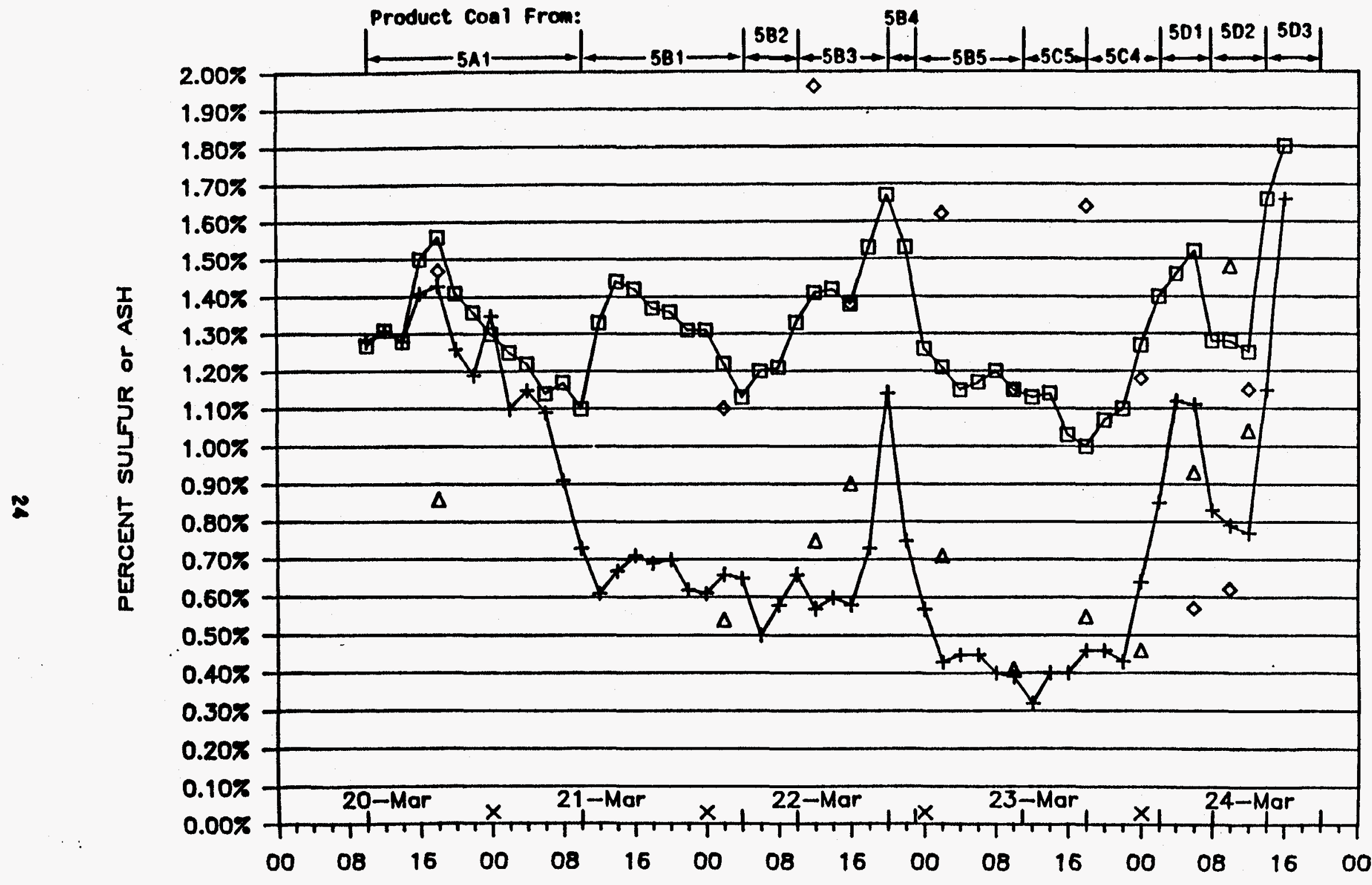

PROD SUL

$+\quad$ PROD ASH

DATE / HOUR

- KILN SUL

$\triangle \quad$ KILN ASH 


\section{Run Conditton Summary}

Operat Ing Run No. 5, 3/20-3/24/89

\begin{tabular}{|c|c|c|c|c|c|c|c|c|c|}
\hline & $\begin{array}{l}\text { Operat ir } \\
\text { Span for }\end{array}$ & $\begin{array}{l}n g T \text { tme } \\
r \quad k+1 n^{*}\end{array}$ & & & & & & & \\
\hline $\begin{array}{l}\text { Run } \\
\text { No. }\end{array}$ & $\begin{array}{l}\text { Date } \\
\text { From/To }\end{array}$ & $\begin{array}{c}\text { T1me } \\
\text { From/To }\end{array}$ & $\begin{array}{l}\text { Coal } \\
\text { Iype }\end{array}$ & $\begin{array}{l}\text { Caust tc } \\
\text { Iype } \\
\end{array}$ & Caustic:Coa1 & $\begin{array}{l}k+\ln S k \\
\text { Zone } 1\end{array}$ & $\frac{n \text { Temps*: }}{\text { Zone }}$ & Zone & Zone_ 4 \\
\hline 5A1 & $\begin{array}{l}3 / 20 \\
3 / 21\end{array}$ & $\begin{array}{l}0100- \\
0055\end{array}$ & $\begin{array}{l}\text { P1ttsburgh } 18 \\
14 \text { Mesh }\end{array}$ & $\begin{array}{l}\text { NaOH: KOH } \\
(50: 50)\end{array}$ & $2: 1$ & 421 & 410 & 410 & 410 \\
\hline 581 & $3 / 21$ & 0055-1900 & $\begin{array}{l}\text { P1ttsburgh } 8 \\
14 \text { Mesh }\end{array}$ & $\mathrm{NaOH}$ & $2: 1$ & 421 & 410 & 410 & 410 \\
\hline $5 B 2$ & $\begin{array}{l}3 / 21 \\
3 / 22\end{array}$ & $\begin{array}{l}1900- \\
0150\end{array}$ & $\begin{array}{l}\text { Pittsburgh "8 } \\
14 \text { Mesh }\end{array}$ & $\mathrm{NaOH}$ & $2: 1$ & 449 & 410 & 410 & 410 \\
\hline 583 & $3 / 22$ & $0150-1215$ & $\begin{array}{l}\text { P1ttsburgh :8 } \\
14 \text { Mesh }\end{array}$ & $\mathrm{NaOH}$ & $2: 1$ & $\begin{array}{l}\text { Heater } \\
\text { off }\end{array}$ & 449 & 410 & 410 \\
\hline 584 & $3 / 22$ & $1215-1600$ & $\begin{array}{l}\text { Pittsburgh } 18 \\
14 \text { Mesh }\end{array}$ & $\mathrm{NaOH}$ & $2: 1$ & $\begin{array}{l}\text { Heater } \\
\text { off }\end{array}$ & $\begin{array}{l}\text { Heater } \\
\text { off }\end{array}$ & 449 & 410 \\
\hline 5B5 & $\begin{array}{l}3 / 22 \\
3 / 23\end{array}$ & $\begin{array}{l}1600- \\
0230\end{array}$ & $\begin{array}{l}\text { Pittsburgh } 8 \\
14 \text { Mesh }\end{array}$ & $\mathrm{NaOH}$ & $2: 1$ & 427 & 427 & 427 & 427 \\
\hline $5 C 5$ & $3 / 23$ & 0230-1005 & $\begin{array}{l}\text { Kentucky :9 } \\
14 \text { Mesh }\end{array}$ & $\mathrm{NaOH}$ & $2: 1$ & 427 & 427 & 427 & 427 \\
\hline $5 C 4$ & $3 / 23$ & $1005-1800$ & $\begin{array}{l}\text { Kentucky :9 } \\
14 \text { Mesh }\end{array}$ & $\mathrm{NaOH}$ & $2: 1$ & $\begin{array}{l}\text { Heater } \\
\text { Off }\end{array}$ & 427 & 427 & 427 \\
\hline
\end{tabular}

* It has been calculated that it takes -8 hours for the coal to get thru the $k 1$ ln and wash trains. Therefore, when relating product coal collected to kiln conditfons this 8 hour lag t ime must be taken into account.

* Established in later runs that $k+1$ in internal equflibrium process temperature $-20-25^{\circ} \mathrm{C}$ 10wer than external kiln wall skin temperature. 


\section{Run Condition Summary}

Operating Run No. 5, 3/20-3/24/89

(Cont inued)

\begin{tabular}{|c|c|c|c|c|c|c|c|c|c|}
\hline \multirow[b]{2}{*}{$\begin{array}{l}\text { Run } \\
\text { No. }\end{array}$} & \multicolumn{2}{|c|}{$\begin{array}{l}\text { Operat ing Time } \\
\text { Span for } k+1 n^{*}\end{array}$} & \multirow[b]{2}{*}{$\begin{array}{l}\text { Coal } \\
\text { Type }\end{array}$} & \multirow[b]{2}{*}{$\begin{array}{l}\text { Caust ic } \\
\text { Type }\end{array}$} & \multirow[b]{2}{*}{ Caust tc:Coal } & \multirow{2}{*}{\multicolumn{2}{|c|}{ KtIn SkIn Temps** }} & \multirow[b]{2}{*}{$2_{7}^{\circ} \underline{C}$} & \multirow[b]{2}{*}{ Zone 4} \\
\hline & $\begin{array}{l}\text { Date } \\
\text { From/To }\end{array}$ & $\begin{array}{l}\text { Time } \\
\text { From/To }\end{array}$ & & & & & & & \\
\hline 501 & $\begin{array}{l}3 / 23 \\
3 / 24\end{array}$ & $\begin{array}{l}1800- \\
0030\end{array}$ & $\begin{array}{l}\text { Kentucky } 19 \\
14 \text { Mesh }\end{array}$ & $\mathrm{NaOH}$ & $2: 1$ & $\begin{array}{l}\text { Heater } \\
\text { off }\end{array}$ & $\begin{array}{l}\text { Heater } \\
\text { Off }\end{array}$ & 427 & 427 \\
\hline 502 & $3 / 24$ & $0030-0630$ & $\begin{array}{l}\text { Kentucky } 19 \\
14 \text { Mesh }\end{array}$ & $\mathrm{NaOH}$ & $2: 1$ & 399 & 399 & 399 & 399 \\
\hline 503 & $3 / 24$ & $0630-1220$ & $\begin{array}{l}\text { Kentucky } 19 \\
14 \text { Mesh }\end{array}$ & $\mathrm{NaOH}$ & $2: 1$ & 371 & 371 & 371 & 371 \\
\hline
\end{tabular}

* It has been calculated that it takes -8 hours for the coal to get thru the $k 1$ in and wash trains. Therefore, when relating product coal collected to $k+1 n$ conditions this 8 hour lag time must be taken into account.

** Established in later runs that ktin Internal equilibrium process temperature $-20-25^{\circ} \mathrm{C}$ lower than external kiln wall skin temperature. 
MCL OPERATINQ DATA

RUN NUMBER 6: APR 03-07,1989

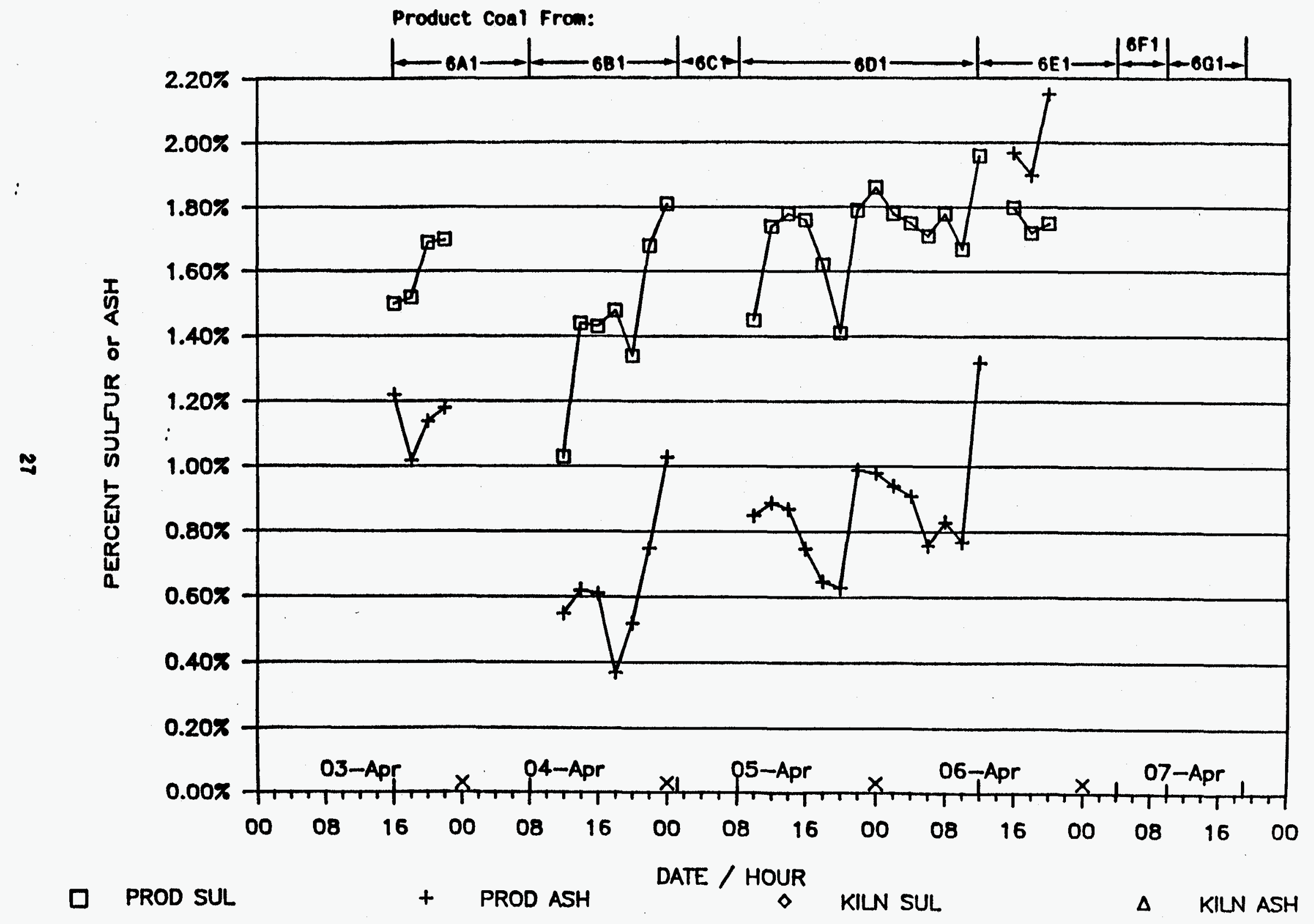




\section{Rül Condition Summary}

Operat In! Run No. 6, 4/03-4/07/89

\begin{tabular}{|c|c|c|c|c|c|c|c|}
\hline \multirow[b]{2}{*}{$\begin{array}{l}\text { Run } \\
\text { No. }\end{array}$} & \multicolumn{2}{|c|}{$\begin{array}{l}\text { Operat Ing Time } \\
\text { Span for } k i 1 n^{*}\end{array}$} & \multirow[b]{2}{*}{$\begin{array}{l}\text { Coa1 } \\
\text { Type }\end{array}$} & \multirow[b]{2}{*}{$\begin{array}{l}\text { Caustic } \\
\text { Type }\end{array}$} & \multirow[b]{2}{*}{ Caustic:Coal } & \multirow[b]{2}{*}{$\begin{array}{l}\text { Ktln Feed } \\
\text { Rate } 1 \mathrm{bs} / \mathrm{hr}\end{array}$} & \multirow[b]{2}{*}{$\begin{array}{l}\text { Klln Skin } \\
\text { Iemps }{ }^{*}{ }^{* *}\end{array}$} \\
\hline & $\begin{array}{l}\text { Date } \\
\text { From/To }\end{array}$ & $\begin{array}{l}\text { T1me } \\
\text { From/To }\end{array}$ & & & & & \\
\hline 6A1 & $\begin{array}{l}4 / 03 \\
4 / 04\end{array}$ & $\begin{array}{l}0400- \\
0000\end{array}$ & $\begin{array}{l}\text { Pittsburgh "8 } \\
14 \text { Mesh }\end{array}$ & $\mathrm{NaOH}$ & $1: 1$ & 30 & Al1 zones e $427^{\circ} \mathrm{C}$ \\
\hline 6B1 & 4/04 & $0130-1645$ & $\begin{array}{l}\text { P1ttsburgh } 18 \\
14 \text { Mesh }\end{array}$ & $\mathrm{NaOH}$ & $1.5: 1$ & 30 & Al1 zones $1427^{\circ} \mathrm{C}$ \\
\hline oc1 & 4/04 & $1645-2130$ & $\begin{array}{l}\text { Pittsburgh } 18 \\
14 \text { Mesh }\end{array}$ & $\mathrm{MaOH}$ & $1: 1$ & 30 & A11 zones $410^{\circ} \mathrm{C}$ \\
\hline 601 & $\begin{array}{l}4 / 05 \\
4 / 06\end{array}$ & $\begin{array}{l}0000- \\
0400\end{array}$ & $\begin{array}{l}\text { Pittsburgh } 18 \\
14 \text { Mesh }\end{array}$ & $\mathrm{NaOH}$ & $1.5: 1$ & 30 & All zones $410^{\circ} \mathrm{C}$ \\
\hline 6E1 & 4/06 & $0515-1900$ & $\begin{array}{l}\text { P1ttsburgh } 18 \\
14 \text { Mesh }\end{array}$ & $\begin{array}{l}\text { NaOH: KOH } \\
(50: 50)\end{array}$ & $1: 1$ & 30 & All zones e $427^{\circ} \mathrm{C}$ \\
\hline 6F1 & $\begin{array}{l}9 / 06 \\
4 / 07\end{array}$ & $\begin{array}{l}1900- \\
0200\end{array}$ & $\begin{array}{l}\text { Pittsburgh } \\
14 \text { Mesh }\end{array}$ & $\begin{array}{l}\text { NaOH: KOH } \\
\text { (50:50) }\end{array}$ & $1: 1$ & 50 & All zones $e 427^{\circ} \mathrm{C}$ \\
\hline 601 & $4 / 07$ & $0200-1130$ & $\begin{array}{l}\text { Pittsburgh } 18 \\
14 \text { Mesh }\end{array}$ & $\begin{array}{l}\text { NaOH:KOH } \\
(50: 50)\end{array}$ & $1: 1$ & 40 & $A 11$ zones $427^{\circ} \mathrm{C}$ \\
\hline
\end{tabular}

- It has been calculated that it takes -8 hours for the coal to get thru the $k+1$ in and wash trains. Therefore, when relating product coal collected to $k+1$ ln conditions this 8 hour lag time must be taken into account.

* Established in later runs that $k 11 n$ internal equilibrium process temperature $-20-25^{\circ} \mathrm{C}$ lower than external $k+1 n$ wall skin temperature. 
MCL OPERATING DATA

RUN NUMBER 7: MAY 01-05, 1989

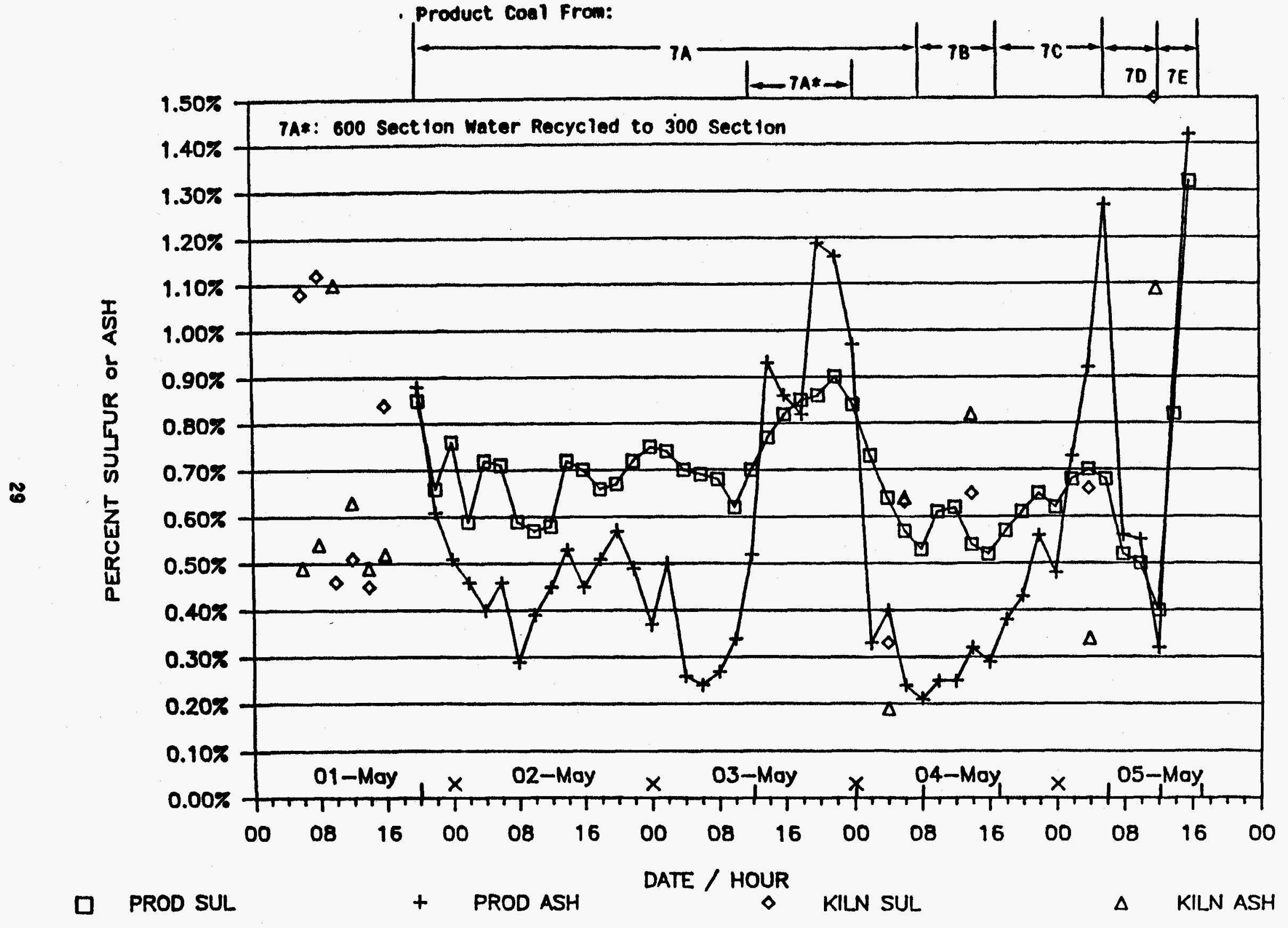


Run Condition Summary

Operating Run No. 7, 5/01-5/05/89

\begin{tabular}{|c|c|c|c|c|c|c|}
\hline \multirow[b]{2}{*}{$\begin{array}{l}\text { Run } \\
\text { No. }\end{array}$} & \multicolumn{2}{|c|}{$\begin{array}{l}\text { Operating } T \text { Ime } \\
\text { Span for } k+1 n^{*}\end{array}$} & \multirow[b]{2}{*}{$\begin{array}{l}\text { Coal } \\
\text { Iype }\end{array}$} & \multirow[b]{2}{*}{$\begin{array}{l}\text { Caust ic } \\
\text { Iype }\end{array}$} & \multirow[b]{2}{*}{ Caust 1c:Coal } & \multirow[b]{2}{*}{ KIIn Sk1n Temps** } \\
\hline & $\begin{array}{l}\text { Date } \\
\text { From/To }\end{array}$ & $\begin{array}{l}\text { Time } \\
\text { From/To }\end{array}$ & & & & \\
\hline $7 A$ & $\begin{array}{l}5 / 01 \\
5 / 03\end{array}$ & $\begin{array}{l}0000- \\
2330\end{array}$ & $\begin{array}{l}\text { Kentucky } 19 \\
14 \text { Mesh }\end{array}$ & $\begin{array}{l}\mathrm{NaOH}: \mathrm{KOH} \\
(50: 50), \text { fresh }\end{array}$ & $2.5: 1$ & Al1 zones e $427^{\circ} \mathrm{C}$ \\
\hline 78 & $5 / 04$ & $0000-0930$ & $\begin{array}{l}\text { Kentucky :9 } \\
14 \text { Mesh }\end{array}$ & $\begin{array}{l}\mathrm{NaOH}: \mathrm{KOH} \\
(50: 50) \text {, fresh }\end{array}$ & $3: 1$ & All zones e $427^{\circ} \mathrm{C}$ \\
\hline $7 C$ & $5 / 04$ & $0930-2250$ & $\begin{array}{l}\text { Pittsburgh } \$ 8 \\
6 \text { Mesh }\end{array}$ & $\begin{array}{l}\mathrm{NaOH}: \mathrm{KOH} \\
(50: 50) \text {, fresh }\end{array}$ & $3: 1$ & All zones e $427^{\circ} \mathrm{C}$ \\
\hline 70 & $\begin{array}{l}5 / 04 \\
5 / 05\end{array}$ & $\begin{array}{l}2330- \\
0730\end{array}$ & $\begin{array}{l}\text { Pittsburgh } 18 \\
14 \text { Mesh }\end{array}$ & $\begin{array}{l}\text { NaOH: } \mathrm{KOH} \\
(50: 50) \text {, partially } \\
\text { regenerated }\end{array}$ & $2: 1$ & All zones $427^{\circ} \mathrm{C}$ \\
\hline TE & $5 / 05$ & $0730-1230$ & $\begin{array}{l}\text { Pittsburgh } 18 \\
14 \text { Mesh }\end{array}$ & $\begin{array}{l}\text { NaOH:KOH } \\
(50: 50), \text { fresh }\end{array}$ & $2: 1$ & $A 11$ zones $9427^{\circ} \mathrm{C}$ \\
\hline
\end{tabular}

* It has been calculated that it takes -8 hours for the coal to get thru the $k+1 n$ and wash trains. Therefore, when relating product coal collected to kiln conditions this 8 hour lag time must be taken into account.

* Internally measured temperature $-20-25^{\circ} \mathrm{C}$ lower. 
MCL OPERATINQ DATA

RUN NUMBER B; MAY 15-19,1989

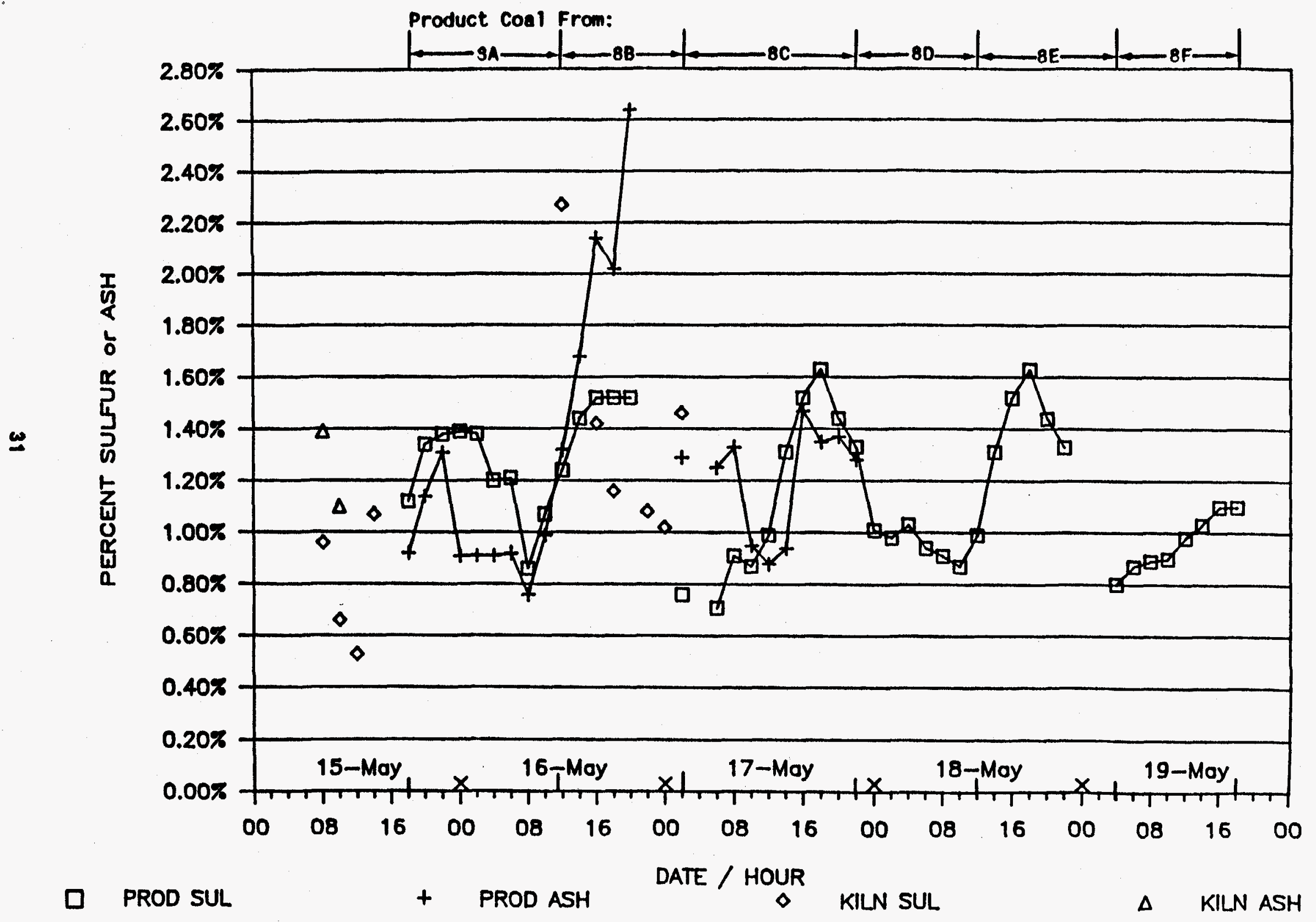


Run_Cond1t Ion Summary

Operating Run No. 8, 5/15-5/19/89

\begin{tabular}{|c|c|c|c|c|c|c|}
\hline \multirow[b]{2}{*}{$\begin{array}{l}\text { Run } \\
\text { No. }\end{array}$} & \multicolumn{2}{|c|}{$\begin{array}{l}\text { Operating Time } \\
\text { Span for } K i l n^{*}\end{array}$} & \multirow[b]{2}{*}{$\begin{array}{l}\text { Coal } \\
\text { Iype }\end{array}$} & \multirow[b]{2}{*}{$\begin{array}{l}\text { Caust tc } \\
\text { Iype }\end{array}$} & \multirow[b]{2}{*}{ Caust 1c:Coa1 } & \multirow[b]{2}{*}{ kiln Skin Temps: } \\
\hline & $\begin{array}{l}\text { Date } \\
\text { Erom/To }\end{array}$ & $\begin{array}{l}\text { Time } \\
\text { From/to }\end{array}$ & & & & \\
\hline $8 \mathbf{A}$ & $\begin{array}{l}5 / 15 \\
5 / 16\end{array}$ & $\begin{array}{l}0200- \\
0300\end{array}$ & $\begin{array}{l}\text { Pittsburgh } 18 \\
14 \text { Mesh }\end{array}$ & $\begin{array}{l}\mathrm{NaOH}: \mathrm{KOH} \\
(50: 50)\end{array}$ & $1.5: 1$ & Al1 zones $427^{\circ} \mathrm{C}$ \\
\hline 8B & $5 / 16$ & $0300-1820$ & $\begin{array}{l}\text { P1ttsburgh } 8 \\
6 \text { Mesh }\end{array}$ & $\begin{array}{l}\mathrm{NaOH}: \mathrm{KOH} \\
(50: 50)\end{array}$ & $1.5: 1$ & All zones $1427^{\circ} \mathrm{C}$ \\
\hline $8 \mathrm{C}$ & $\begin{array}{l}5 / 16 \\
5 / 17\end{array}$ & $\begin{array}{l}1820- \\
1300\end{array}$ & $\begin{array}{l}\text { P1ttsburgh } 18 \\
6 \text { Mesh }\end{array}$ & $\mathrm{NaOH}$ only & $1.5: 1$ & All zones e $427^{\circ} \mathrm{C}$ \\
\hline 80 & $\begin{array}{l}5 / 17 \\
5 / 18\end{array}$ & $\begin{array}{l}1300- \\
0415\end{array}$ & $\begin{array}{l}\text { Pittsburgh } 8 \\
6 \text { Mesh }\end{array}$ & NaOH only & $1.5: 1$ & Al1 zones $4442^{\circ} \mathrm{C}$ \\
\hline 8E & $5 / 18$ & $0415-2000$ & $\begin{array}{l}\text { Pittsburgh } 8 \\
6 \text { Mesh }\end{array}$ & $\mathrm{NaOH}$ only & $1.5: 1$ & Al1 zones e $412^{\circ} \mathrm{C}$ \\
\hline $8 \mathrm{~F}$ & $\begin{array}{l}5 / 18 \\
5 / 19\end{array}$ & $\begin{array}{l}2000- \\
1300\end{array}$ & $\begin{array}{l}\text { P1ttsburgh } 18 \\
14 \text { Mesh }\end{array}$ & $\mathrm{NaOH}$ only & $2: 1$ & All zones e $412^{\circ} \mathrm{C}$ \\
\hline
\end{tabular}

* It has been calculated that it takes -8 hours for the coal to get thru the kiln and wash trains. Therefore, when relating product coal collected to kiln conditions this 8 hour lag time must be taken into account.

** Internally measured temperature $-20-25^{\circ} \mathrm{C}$ lower. 


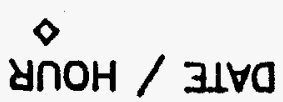

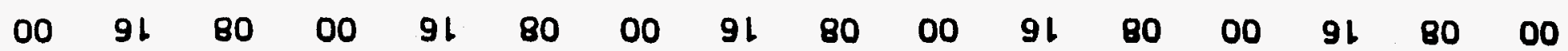

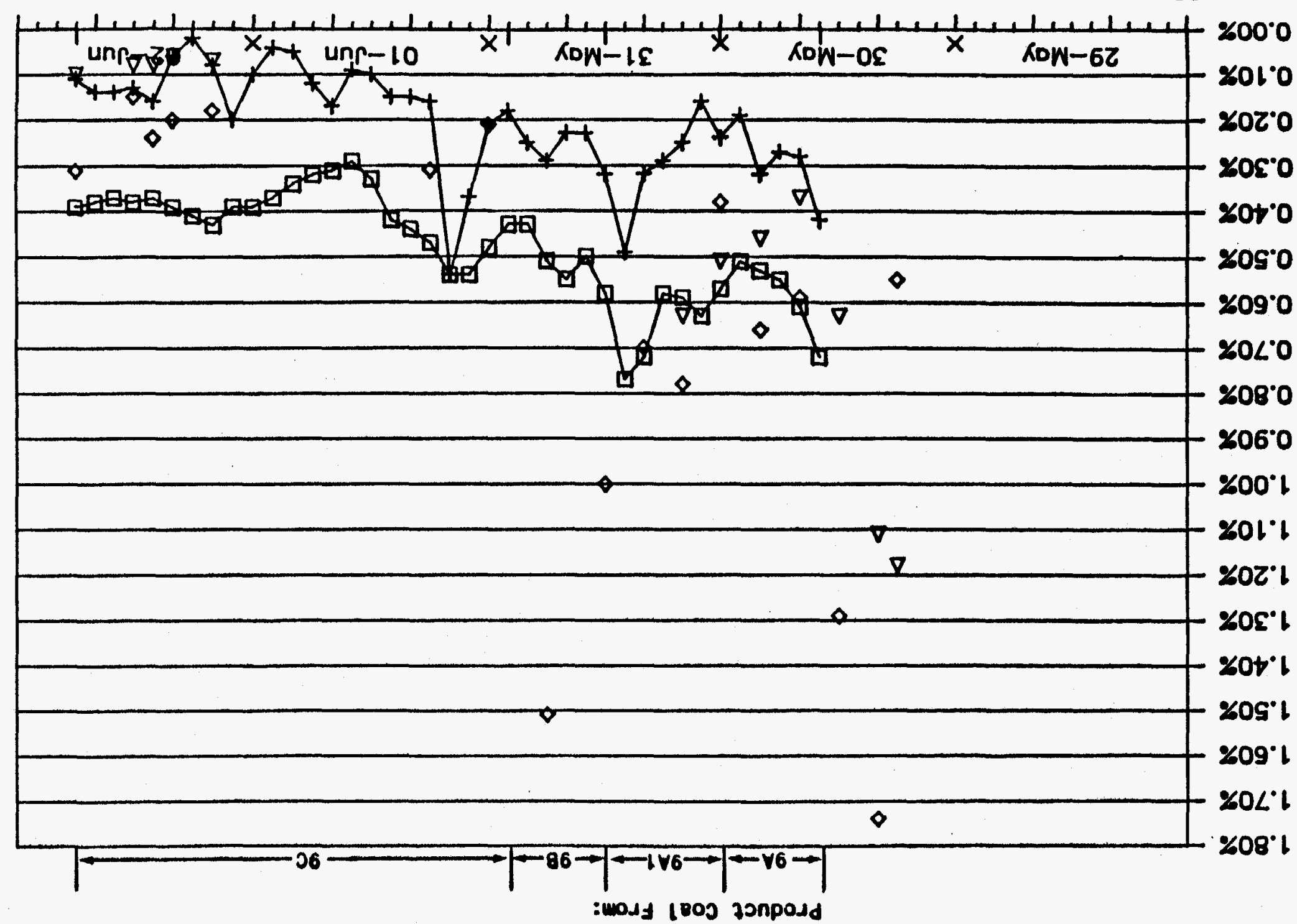


Run_Condit ton Summary

Operating Run No. 9, 5/30-6/2/89

Operating Time

$\frac{\text { Span for } K I \mathrm{ln} *}{\text { Date } T \text { ime }}$

Run

Coal

Iype

Caustic

From/To From/To

Pittsburgh 18

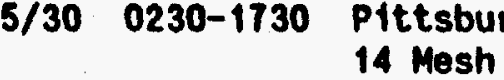

Type

Caust 1c:Coal

Kiln Sk+n Temps*:

$9 A$

$\mathrm{NaOH}: \mathrm{KOH}$

$2.5: 1$

All zones $427^{\circ} \mathrm{C}$

9A1

$5 / 30 \quad 1730$ - P1ttsburgh 18

$5 / 31$

0430

14 Mesh

$(50: 50)$, fresh

$2.5: 1$

All zones $438^{\circ} \mathrm{C}$

9B

5/31 0530-1115 P1ttsburgh 18

14 Mesh

$(50: 50)$, fresh

$\mathrm{NaOH}: \mathrm{KOH}$

(50:50),

regenerated

9C

$5 / 31$ $6 / 02$

1315 - P1ttsburgh 18 1530 14 Mesh

$\mathrm{NaOH}$ on 1 y, fresh

* It has been calculated that $1 t$ takes -8 hours for the coal to get thru the $k 1$ in and wash trains. Therefore, when relating product coal collected to $k+1 n$ conditions this 8 hour lag time must be taken into account.

* Internally measured temperature -20-25 ${ }^{\circ} \mathrm{C}$ lower. 
4.2.2 Volatiles and Heat Content Data for Product Coa 1 as a Function of Coal Exit Time 
MCL OPERATING DATA

RUN NUMBER S1: DEC 12-16,1988

Product Coal From:

S10

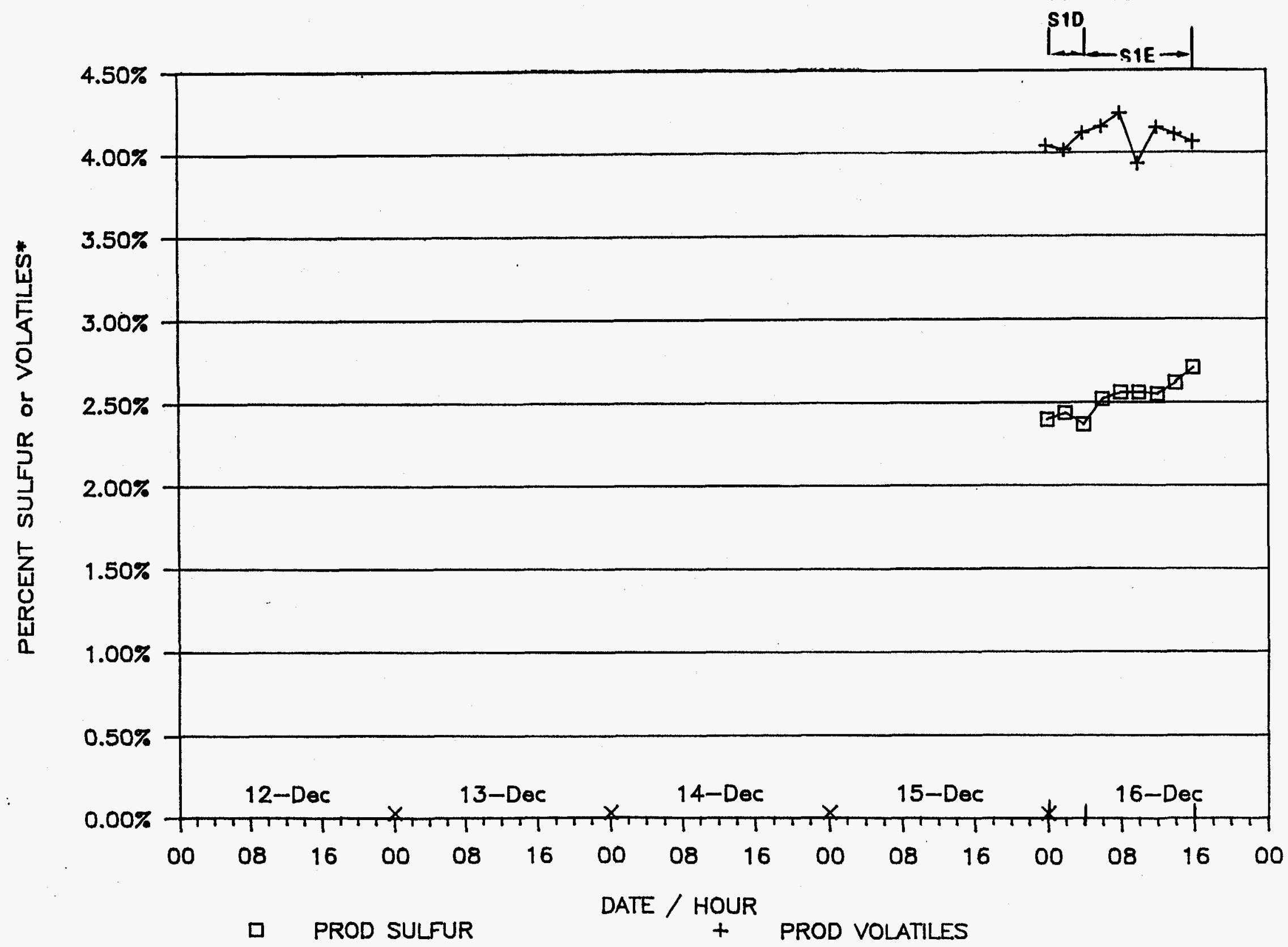

* Volatiles reading must be mitifilied by a fetor of 10 . 


\section{Run Condition Summary}

Shakedown Run No. $1,12 / 12-12 / 16 / 88$

\begin{tabular}{|c|c|c|c|c|c|c|}
\hline \multirow[b]{2}{*}{$\begin{array}{l}\text { Run } \\
\text { No. }\end{array}$} & \multicolumn{2}{|c|}{$\begin{array}{l}\text { Operating Time } \\
\text { Span for } k+1 n^{*}\end{array}$} & \multirow[b]{2}{*}{$\begin{array}{l}\text { Coa } 1 \\
\text { Iype }\end{array}$} & \multirow[b]{2}{*}{$\begin{array}{l}\text { Caust ic } \\
\text { Type }\end{array}$} & \multirow[b]{2}{*}{ Caust Ic:Coal } & \multirow[b]{2}{*}{$\begin{array}{l}\text { Kiln Skin } \\
\text { Temps }{ }^{\circ} C^{* *}\end{array}$} \\
\hline & $\begin{array}{l}\text { Date } \\
\text { From/To }\end{array}$ & $\begin{array}{c}\text { Time } \\
\text { From/To }\end{array}$ & & & & \\
\hline S1A & $\begin{array}{l}12 / 12 \\
12 / 13\end{array}$ & $\begin{array}{l}0800- \\
1200\end{array}$ & $\begin{array}{l}\text { Pittsburgh } \# 8 \\
14 \text { Mesh }\end{array}$ & $\begin{array}{l}\mathrm{NaOH}: \mathrm{KOH} \\
(50: 50)\end{array}$ & $1: 1$ & 380 \\
\hline SIB & $12 / 13$ & $1200-1500$ & $\begin{array}{l}\text { Pittsburgh } \# 8 \\
14 \text { Mesh }\end{array}$ & $\begin{array}{l}\text { NaOH:KOH } \\
(50: 50)\end{array}$ & $1: 1$ & 290 \\
\hline sic & $12 / 13$ & $1700-2100$ & $\begin{array}{l}\text { P1ttsburgh } \# 8 \\
14 \text { Mesh }\end{array}$ & $\begin{array}{l}\mathrm{NaOH}: \mathrm{KOH} \\
(50: 50)\end{array}$ & $1: 1$ & 335 (Zone 1), - 315 (Zones 2-4) \\
\hline S1D & $\begin{array}{l}12 / 13 \\
12 / 15\end{array}$ & $\begin{array}{l}2200- \\
2100\end{array}$ & $\begin{array}{l}\text { Pittsburgh } \$ 8 \\
14 \text { Mesh }\end{array}$ & $\begin{array}{l}\text { NaOH:KOH } \\
(50: 50)\end{array}$ & $1: 1$ & 350 (Zone 1 ), -330 (Zones 2-4) \\
\hline SIE & $\begin{array}{l}12 / 15 \\
12 / 16\end{array}$ & $\begin{array}{l}2200- \\
0800\end{array}$ & $\begin{array}{l}\text { Pittsburgh } \$ 8 \\
14 \text { Mesh }\end{array}$ & $\begin{array}{l}\mathrm{NaOH}: \mathrm{KOH} \\
(50: 50)\end{array}$ & $1: 1$ & $360--100 \bullet 12 / 16,0800$ \\
\hline
\end{tabular}

* It has been calculated that it takes -8 hours for the coal to get thru the ktin and wash trains. Therefore, when relating product coal collected to kiln conditions this 8 hour lag time must be taken into account.

** Established in later runs that $k i l n$ internal equilibrium process temperature $-20-25^{\circ} \mathrm{C}$ lower than external kiln wall skin temperature. 
MCL OPERATING DATA

RUN 'NUMBER 1; JAN 23-27, 1989

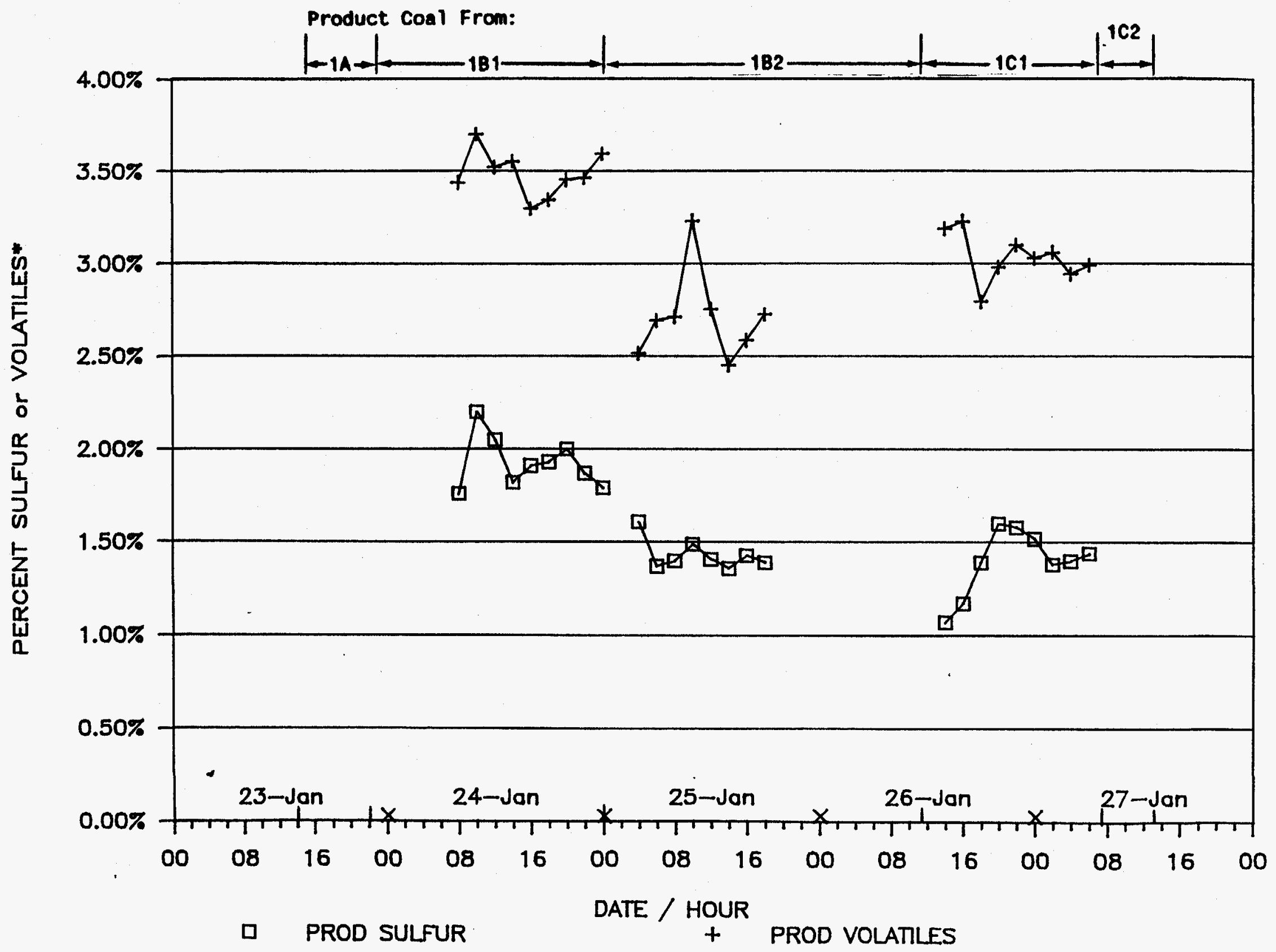

- Volatiles reeding must be miltiplind by a factor of 10. 
Run Condition Summary

Operating Run No. 1, 1/23-1/27/89

\begin{tabular}{|c|c|c|c|c|c|c|}
\hline \multirow[b]{2}{*}{$\begin{array}{l}\text { Run } \\
\text { No. }\end{array}$} & \multicolumn{2}{|c|}{$\begin{array}{l}\text { Operating Time } \\
\text { Span for Kiln* }\end{array}$} & \multirow[b]{2}{*}{$\begin{array}{l}\text { Coal } \\
\text { Iype }\end{array}$} & \multirow[b]{2}{*}{$\begin{array}{l}\text { Caustic } \\
\text { Type }\end{array}$} & \multirow[b]{2}{*}{ Caustic:Coal } & \multirow[b]{2}{*}{ Kiln Skin Temps ${ }^{\circ} \mathrm{C}^{* *}$} \\
\hline & $\begin{array}{l}\text { Date } \\
\text { From/To }\end{array}$ & $\begin{array}{l}\text { Time } \\
\text { From/To }\end{array}$ & & & & \\
\hline $1 A$ & $1 / 23$ & $0400-1430$ & $\begin{array}{l}\text { Pittsburgh } \# 8 \\
14 \text { Mesh }\end{array}$ & $\begin{array}{l}\mathrm{NaOH}: \mathrm{KOH} \\
(50: 50)\end{array}$ & $1: 1$ & 390 \\
\hline 1B1 & $\begin{array}{l}1 / 23 \\
1 / 24\end{array}$ & $\begin{array}{l}1430- \\
1630\end{array}$ & $\begin{array}{l}\text { Pittsburgh } 18 \\
14 \text { Mesh }\end{array}$ & $\begin{array}{l}\mathrm{NaOH}: \mathrm{KOH} \\
(50: 50)\end{array}$ & $1.5: 1$ & 390 \\
\hline $1 \mathrm{B2}$ & $\begin{array}{l}1 / 24 \\
1 / 26\end{array}$ & $\begin{array}{l}1630- \\
0300\end{array}$ & $\begin{array}{l}\text { Pittsburgh } 18 \\
14 \text { Mesh }\end{array}$ & $\begin{array}{l}\mathrm{NaOH}: \mathrm{KOH} \\
(50: 50)\end{array}$ & $1.5: 1$ & 427 \\
\hline $1 C 1$ & $1 / 26$ & $0300-2330$ & $\begin{array}{l}\text { P1ttsburgh } \# 8 \\
14 \text { Mesh }\end{array}$ & $\begin{array}{l}\mathrm{NaOH}: \mathrm{KOH} \\
(50: 50)\end{array}$ & $2: 1$ & 427 \\
\hline $1 \mathrm{C2}$ & $\begin{array}{l}1 / 26 \\
1 / 27\end{array}$ & $\begin{array}{l}2330- \\
1400\end{array}$ & $\begin{array}{l}\text { Pittsburgh } 18 \\
14 \text { Mesh }\end{array}$ & $\begin{array}{l}\mathrm{NaOH}: \mathrm{KOH} \\
(50: 50)\end{array}$ & $2: 1$ & 438 \\
\hline
\end{tabular}

* It has been calculated that it takes -8 hours for the coal to get thru the ktln and wash trains. Therefore, when relating product coal collected to kiln conditlons this 8 hour lag time must be taken into account.

** Established in later runs that $k+1 n$ internal equilibrium process temperature $-20-25^{\circ} \mathrm{C} 10$ wer than external kiln wall skin temperature. 
MCL OPERATING DATA

RUN NUMBER 2: FEB 06-10, 1989

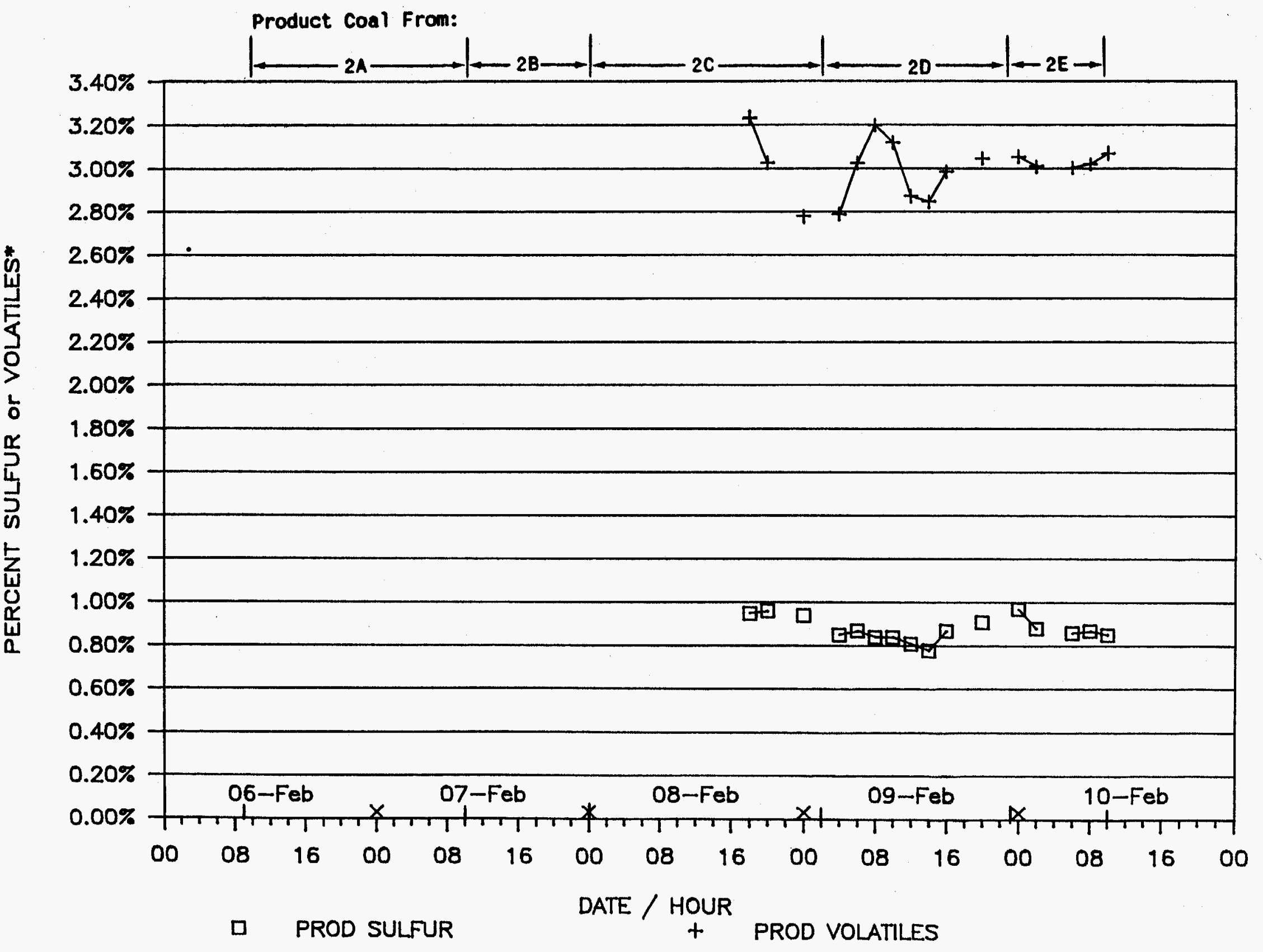

- Volattles reading must be multiolied by a factor of 10. 


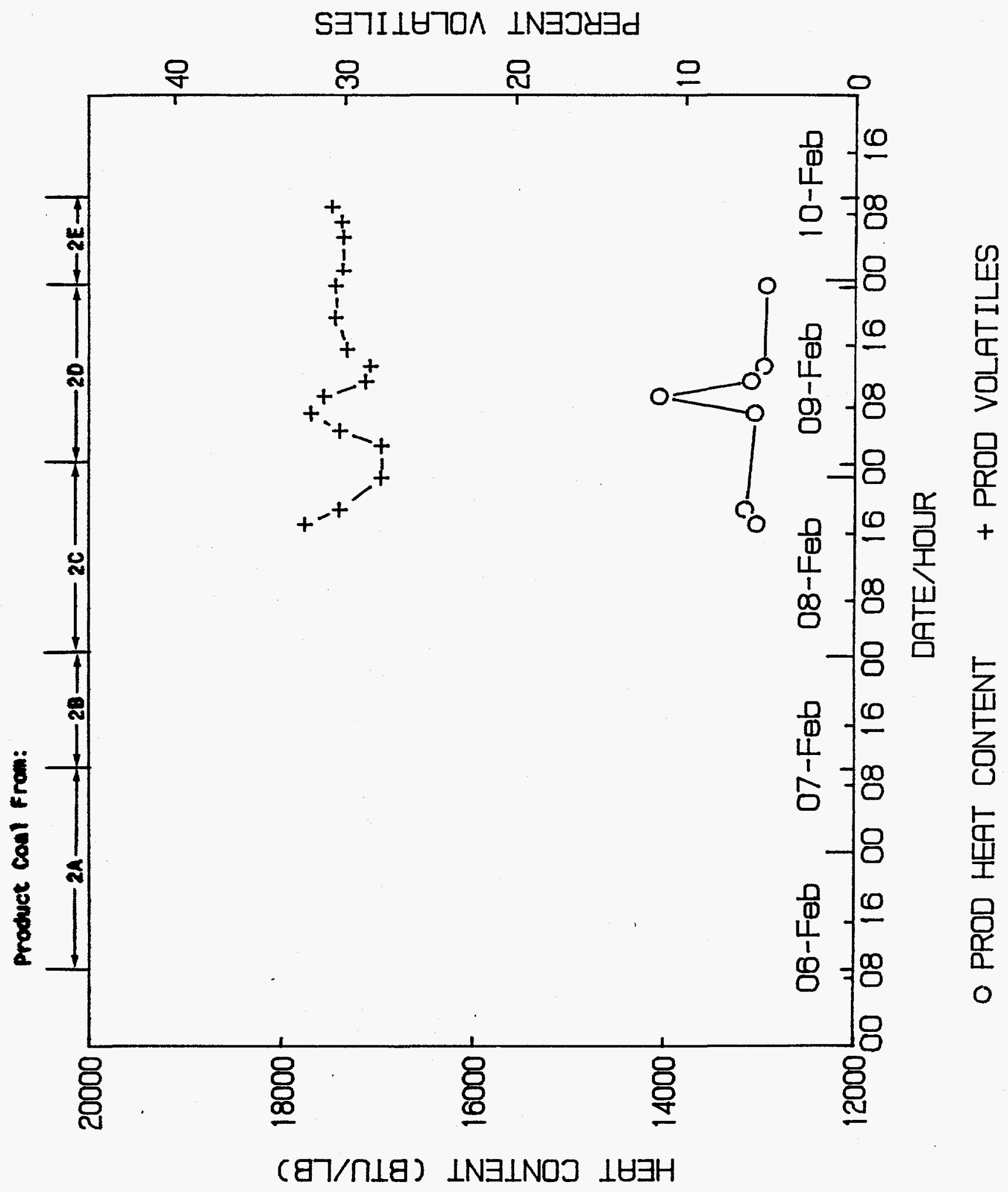




\section{Run Condition Summary}

Operating Run No. 2, 2/06-2/10/89

\begin{tabular}{|c|c|c|c|c|c|c|}
\hline \multirow[b]{2}{*}{$\begin{array}{l}\text { Run } \\
\text { No. }\end{array}$} & \multicolumn{2}{|c|}{$\begin{array}{l}\text { Operating TIme } \\
\text { Span for Kiln* }\end{array}$} & \multirow[b]{2}{*}{$\begin{array}{l}\text { Coal } \\
\text { Type }\end{array}$} & \multirow[b]{2}{*}{$\begin{array}{l}\text { Caustic } \\
\text { Type }\end{array}$} & \multirow[b]{2}{*}{ Caustic:Coal } & \multirow[b]{2}{*}{$\begin{array}{l}\text { Kiln Skin } \\
\text { Temps }{ }^{\circ} \mathrm{C}^{* *} \\
\end{array}$} \\
\hline & $\begin{array}{l}\text { Date } \\
\text { From/To }\end{array}$ & $\begin{array}{l}\text { Time } \\
\text { From/To }\end{array}$ & & & & \\
\hline $2 A$ & $\begin{array}{l}2 / 06 \\
2 / 07\end{array}$ & $\begin{array}{l}0145- \\
0225\end{array}$ & $\begin{array}{l}\text { Pittsburgh } 18 \\
14 \text { Mesh }\end{array}$ & $\begin{array}{l}\mathrm{NaOH}: \mathrm{KOH} \\
(50: 50)\end{array}$ & $2: 1$ & 390 \\
\hline $2 B$ & $2 / 07$ & $0225-1535$ & $\begin{array}{l}\text { Pittsburgh } 18 \\
14 \text { Mesh }\end{array}$ & $\begin{array}{l}\mathrm{NaOH}: \mathrm{KOH} \\
(50: 50)\end{array}$ & $2: 1$ & 390 \\
\hline $2 C$ & $\begin{array}{l}2 / 07 \\
2 / 08\end{array}$ & $\begin{array}{l}1535- \\
1815\end{array}$ & $\begin{array}{l}\text { P1ttsburgh } \# 8 \\
14 \text { Mesh }\end{array}$ & $\begin{array}{l}\mathrm{NaOH}: \mathrm{KOH} \\
(50: 50)\end{array}$ & $2: 1$ & 427 \\
\hline 20 & $\begin{array}{l}2 / 08 \\
2 / 09\end{array}$ & $\begin{array}{l}1815- \\
1500\end{array}$ & $\begin{array}{l}\text { Kentucky } \$ 9 \\
14 \text { Mesh }\end{array}$ & $\begin{array}{l}\mathrm{NaOH}: \mathrm{KOH} \\
(50: 50)\end{array}$ & $2: 1$ & 427 \\
\hline $2 E$ & $\begin{array}{l}2 / 09 \\
2 / 10\end{array}$ & $\begin{array}{l}1500- \\
1300\end{array}$ & $\begin{array}{l}\text { Kentucky } \# 9 \\
14 \text { Mesh }\end{array}$ & $\begin{array}{l}\mathrm{NaOH}: \mathrm{KOH} \\
(50: 50)\end{array}$ & $2: 1$ & 438 \\
\hline
\end{tabular}

* It has been calculated that it takes -8 hours for the coal to get thru the kiln and wash trains. Therefore, when relating product coal collected to kiln conditions this 8 hour lag time must be taken into account.

** Established in later runs that $\mathrm{klln}$ internal equilibrium process temperature $-20-25^{\circ} \mathrm{C}$ 10wer than external kiln wall skin temperature. 
MCL OPERATING DATA

- RUN NUMBER 3: FEB 20-24, 1989

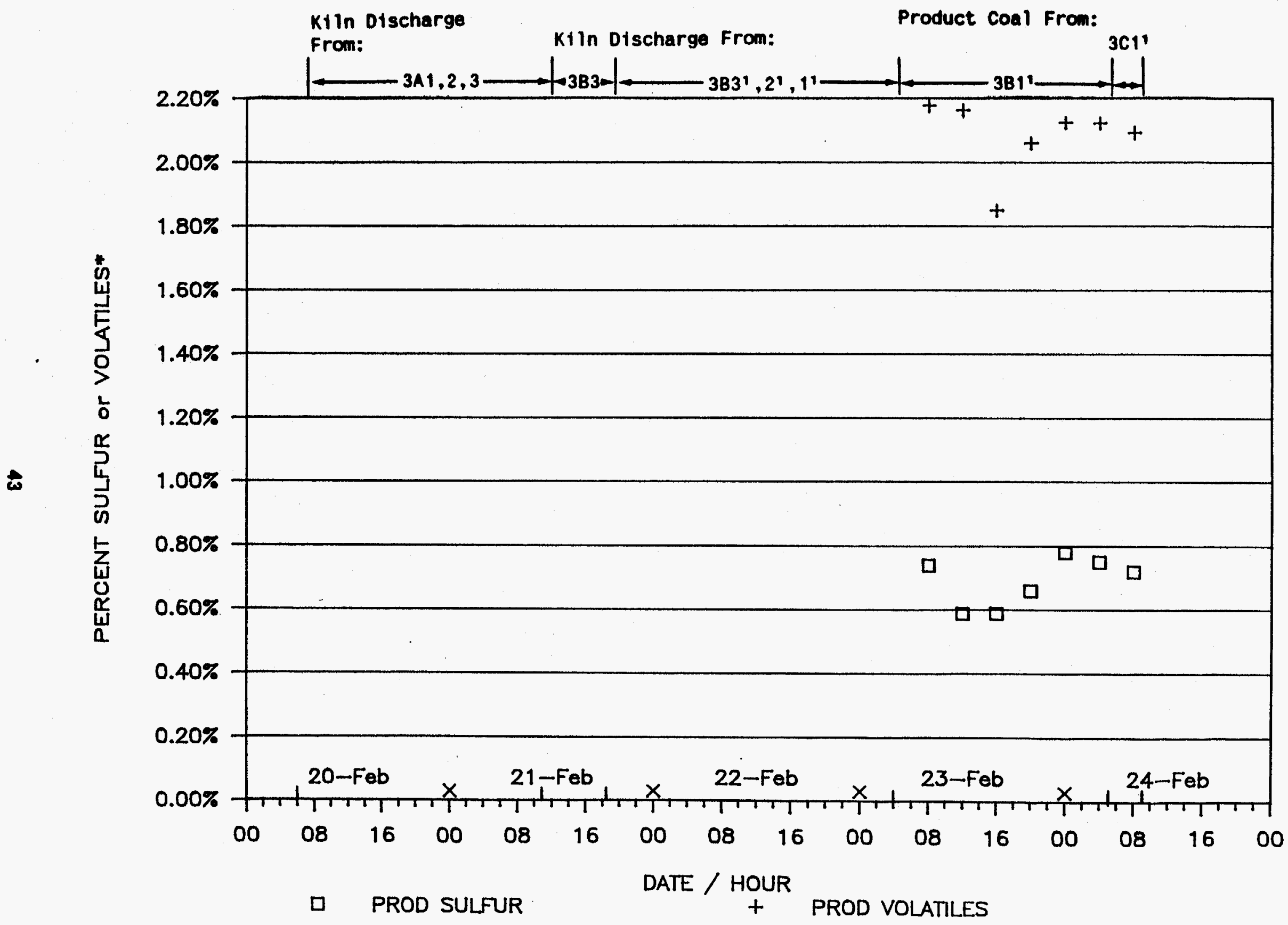

- Volatiles reading must be multiplied by actor of 10. 
MCL OPERATING DATA

RUN NUMBER 3: FEB 20-24, 1989

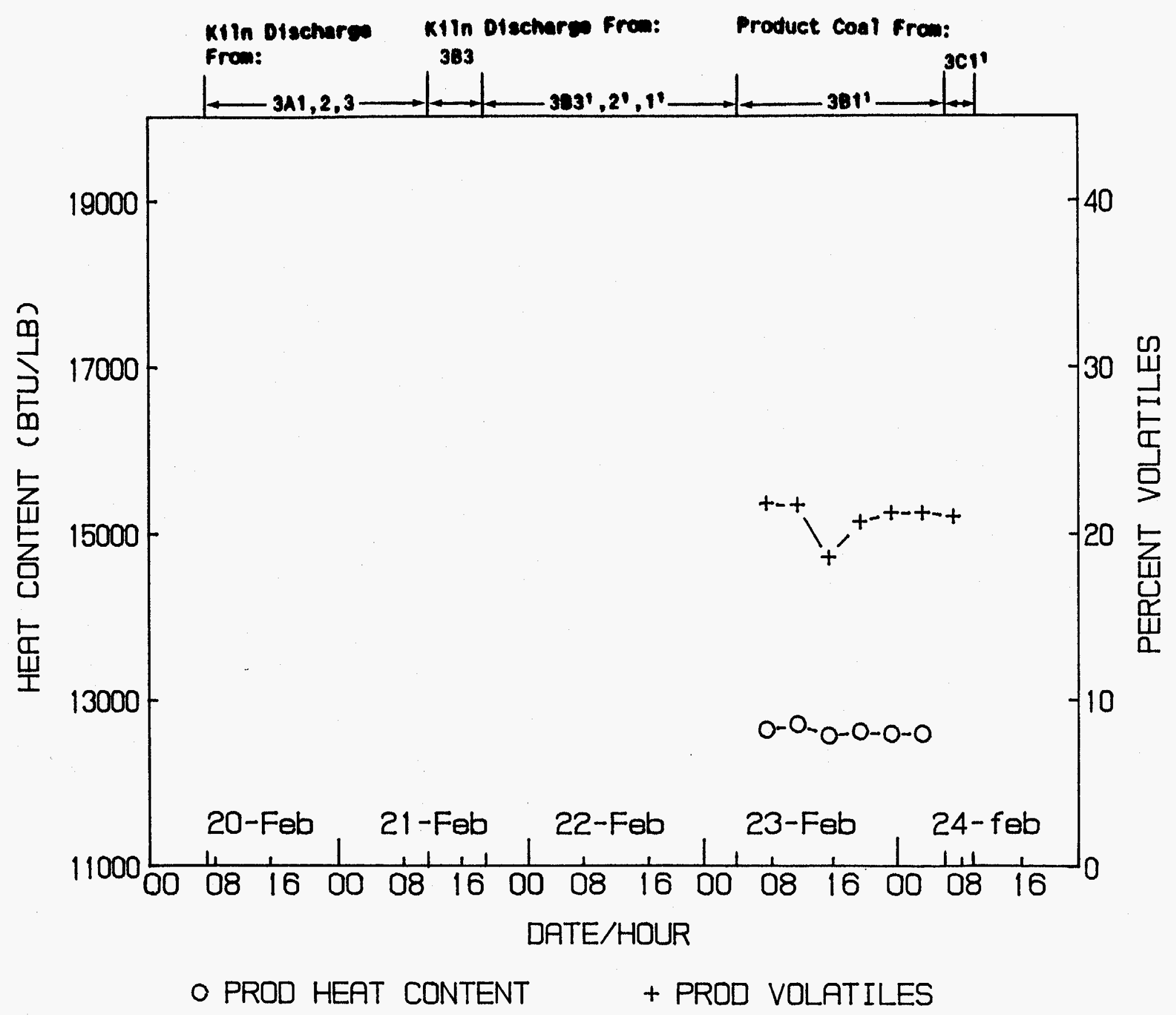


Run Condition Summary

Operating Run No. 3, 2/20-2/24/89

\begin{tabular}{|c|c|c|c|c|c|c|}
\hline $\begin{array}{l}\text { Run } \\
\text { No. }\end{array}$ & $\begin{array}{l}\text { Operatin } \\
\text { Span for } \\
\text { Date } \\
\text { From/To }\end{array}$ & $\begin{array}{c}\text { Ig Time } \\
\text { Kilin* } \\
\text { Time } \\
\text { From/To }\end{array}$ & $\begin{array}{l}\text { Coal } \\
\text { Type }\end{array}$ & $\begin{array}{l}\text { Caust ic } \\
\text { Type }\end{array}$ & Caustic:Coal & $\begin{array}{l}\text { Kiln Skin } \\
\text { Temps }{ }^{\circ} C^{* * * *}\end{array}$ \\
\hline $\begin{array}{l}3 A 1 \\
A 2, A 3 * *\end{array}$ & $\begin{array}{l}2 / 20 \\
2 / 21\end{array}$ & $\begin{array}{l}0130- \\
0650\end{array}$ & $\begin{array}{l}\text { P1ttsburgh } \# 8 \\
14 \text { Mesh }\end{array}$ & $\begin{array}{l}\mathrm{NaOH}: \mathrm{KOH} \\
(50: 50)\end{array}$ & $1: 1$ & 427 \\
\hline 383 & $2 / 21$ & $0720-1520$ & $\begin{array}{l}\text { Pittsburgh } 18 \\
14 \text { Mesh }\end{array}$ & $\begin{array}{l}\mathrm{NaOH}: \mathrm{KOH} \\
(50: 50)\end{array}$ & $2: 1$ & 427 \\
\hline $\begin{array}{l}3 \mathrm{~B}^{1}, \mathrm{~B}^{1} \\
\mathrm{~B}^{1} \neq *: *\end{array}$ & $\begin{array}{l}2 / 21 \\
2 / 24\end{array}$ & $\begin{array}{l}1520- \\
0000\end{array}$ & $\begin{array}{l}\text { Pittsburgh } \# 8 \\
14 \text { Mesh }\end{array}$ & $\begin{array}{l}\mathrm{NaOH}: \mathrm{KOH} \\
(50: 50)\end{array}$ & $2: 1$ & 438 \\
\hline $3 \mathrm{Cl1}$ & $2 / 24$ & $0100-0830$ & $\begin{array}{l}\text { Pittsburgh } \# 8 \\
14 \text { Mesh }\end{array}$ & $\begin{array}{l}\mathrm{NaOH}: \mathrm{KOH} \\
(50: 50)\end{array}$ & $3: 1$ & 438 \\
\hline
\end{tabular}

* It has been calculated that it takes -8 hours for the coal to get thru the $k 1$ ln and wash trains. Therefore, when relating product coal collected to $k+1 n$ conditions this 8 hour lag t tme must be taken into account.

** Tests A1-A3 differ in kiln internal auger setting.

*** Tests B11-B31 differ in k11n internal auger setting.

**** Established in later runs that $k+1 n$ internal equilibrium process temperature $* 20-25^{\circ} \mathrm{C}$ lower than external kiln wall skin temperature. 
MCL OPERATING DATA

RUN NUMBER 4; MAR 06-10, 1989

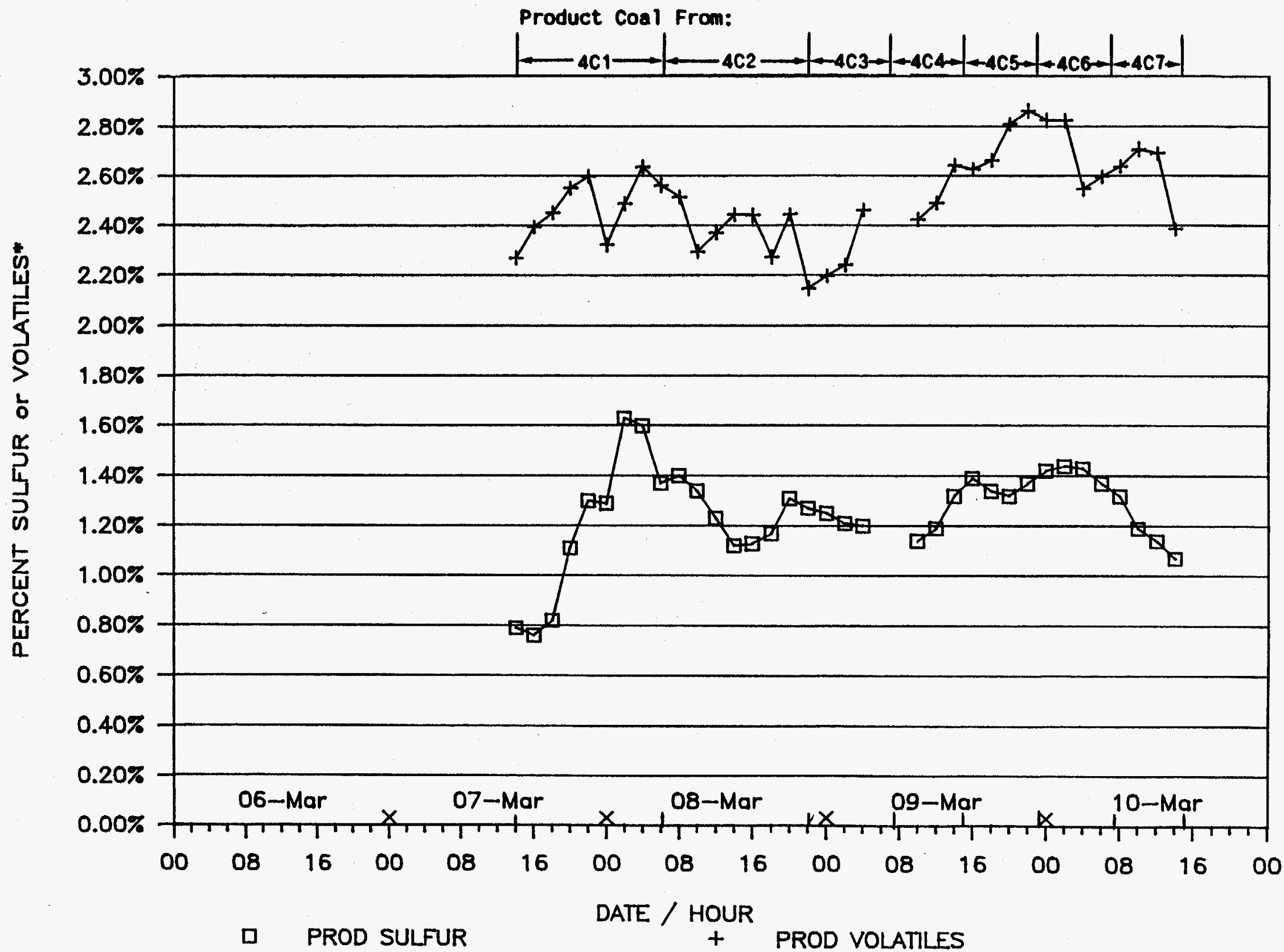

- Volatiles reading must be multiplied by a factor of 10 . 


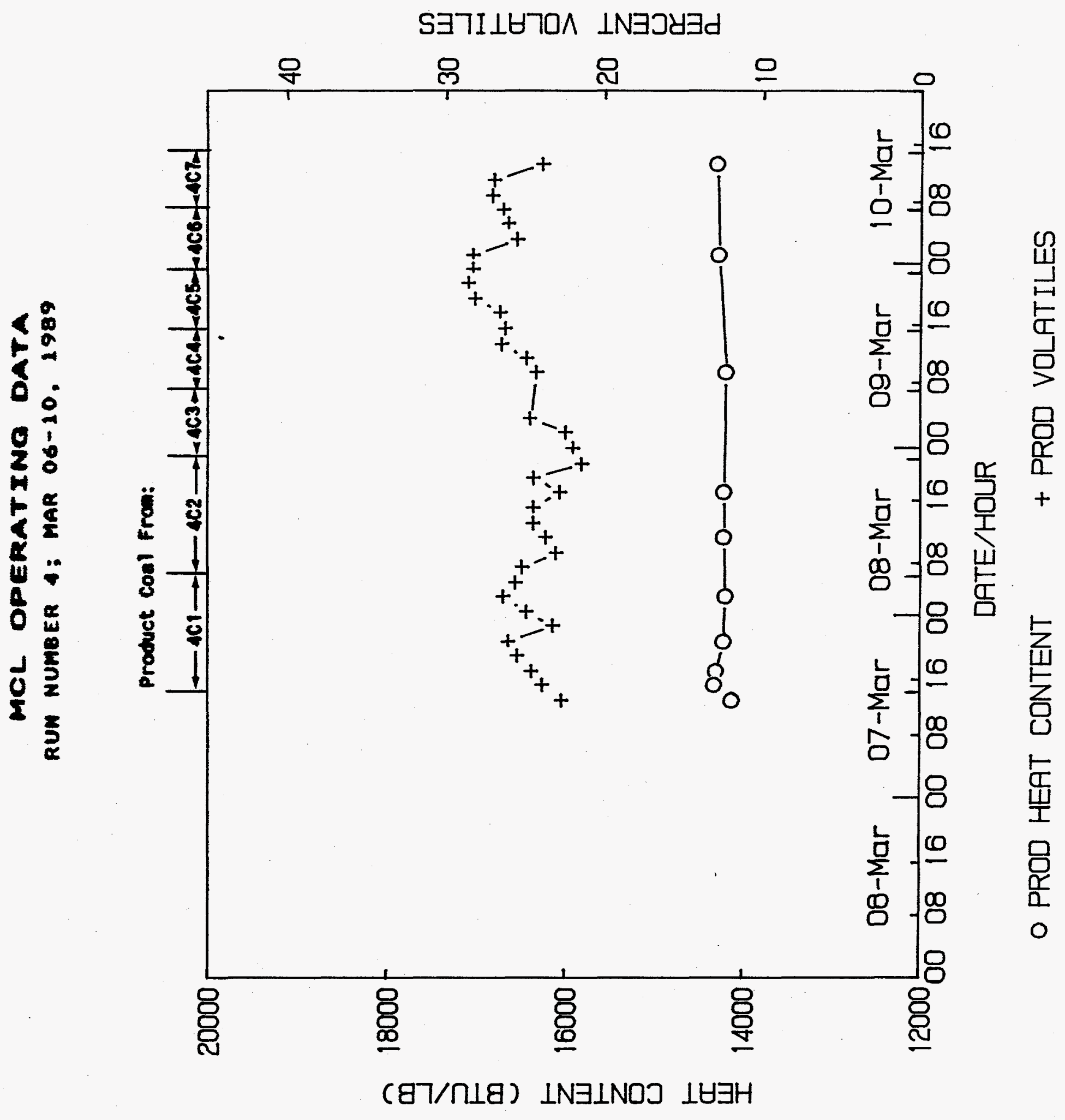




\section{Run Condition Summary}

Operating Run No. 4, 3/06-3/10/89

Operating Time $\frac{\text { Span for } K I l n^{*}}{\text { Date Time }}$

Coa 1

From/To From/To Type

No.

4A

4C1

$3 / 06$

0130-1020

Pittsburgh \#8 14 Mesh

$\begin{array}{ll}3 / 06 & 1630-P i t \text { - Pburgh } \# 8 \\ 3 / 07 & 2200\end{array}$
14 Mesh

$4 C 2$

$4 C 3$

$3 / 072200-$

$3 / 08 \quad 1430$

Pittsburgh $\$ 8$

14 Mesh

$3 / 08 \quad 1540-2300$

Pittsburgh $\$ 8$ 14 Mesh

b $\quad 4 \mathrm{C4}$

$4 C 5$

$4 C 6$

$4 C 7$
$3 / 09$

0100-0700

Pittsburgh $\% 8$

14 Mesh

$3 / 0$

0810-1500

Pittsbur

$3 / 09 \quad 1515-2300$

Pittsburgh \#8

14 Mesh

$3 / 09$, 2315

$3 / 10$
Pittsburgh $\# 8$ 14 Mesh
Caustic

Type

None

$\mathrm{NaOH}$ only

$\mathrm{NaOH}$ only

$\mathrm{NaOH}$ only

$\mathrm{NaOH}$ only

$\mathrm{NaOH}$ only

$\mathrm{NaOH}$ only

$\mathrm{NaOH}$ only
Caust 1c: Coal Kiln Skin Temps**, "

Coal only

$2: 1$

$2: 1$

$2: 1$

$2: 1$

$2: 1$

Off

Heater

off

2:1

Heater

off

$2: 1$

Heater

off

Heater

off

Heater $\quad 449$

off
Zone \#4

410

410

410

410

410

410

421

432

*. It has been calculated that it takes -8 hours for the coal to get thru the kiln and wash trains. Therefore, when relating product coal collected to kiln conditions this 8 hour lag time must be taken into account.

* Established in later runs that kiln internal equilibrium process temperature $-20^{\circ}-25^{\circ} \mathrm{C} 10$ wer than external kiln wall skin temperature. 
MCL OPERATING DATA

RUN NUMBER 5; MAR 20-24, 1989

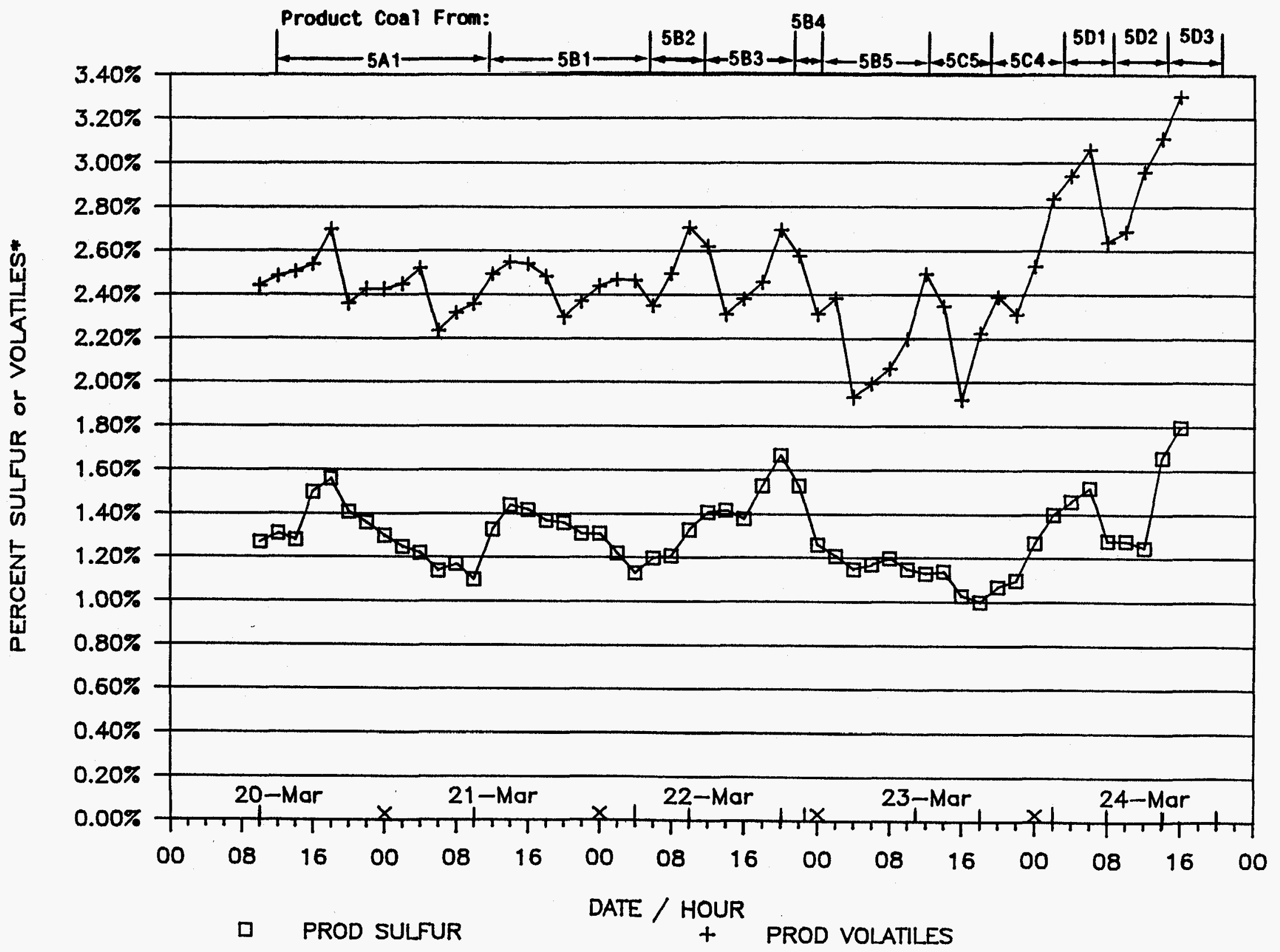

- Volatfles reading must be multiplied by a factor of 10 . 


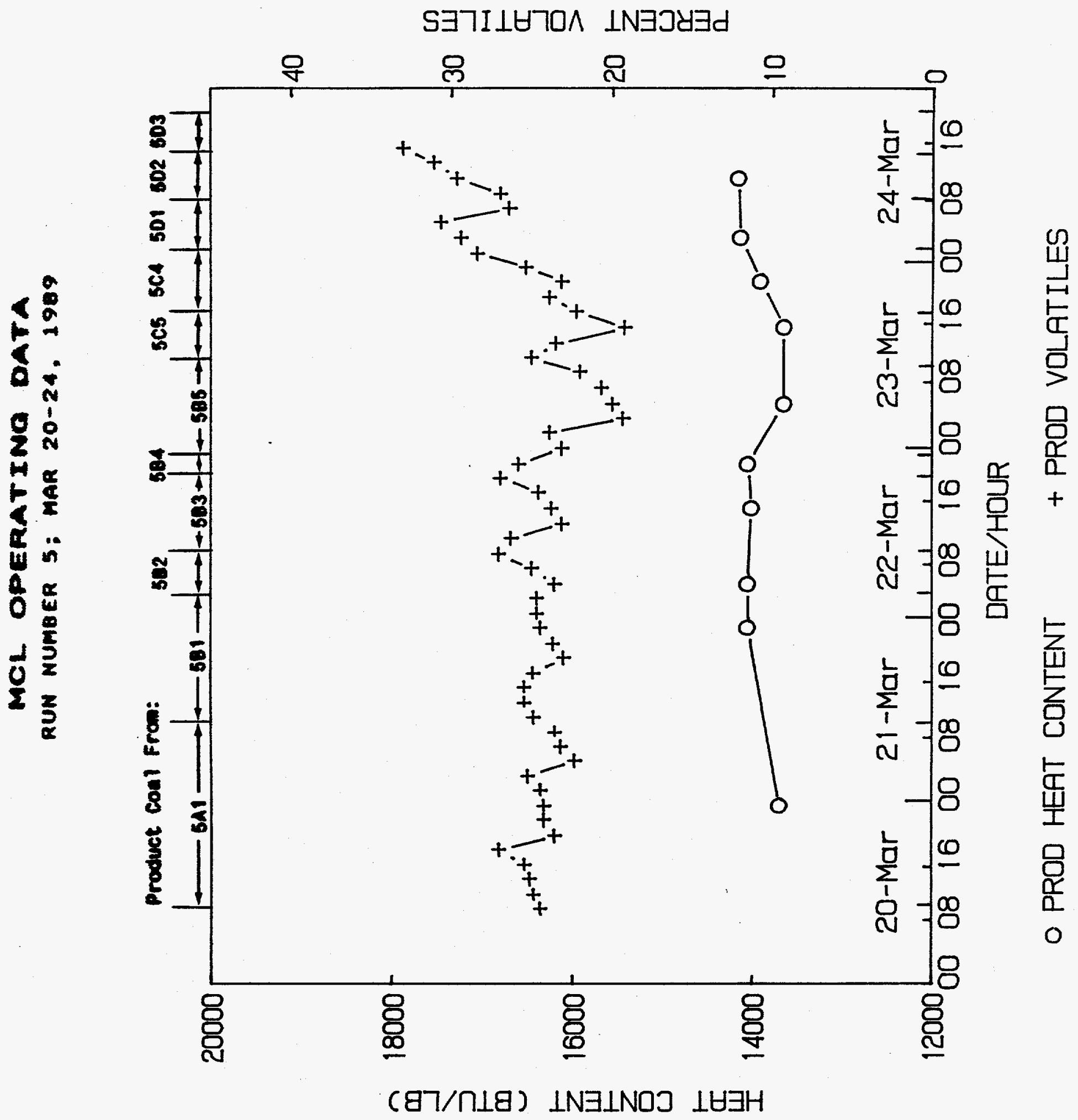


Run Condition Summary

Operating Run No. $5,3 / 20-3 / 24 / 89$

\begin{tabular}{|c|c|c|c|c|c|c|c|c|c|}
\hline \multirow[b]{2}{*}{$\begin{array}{l}\text { Run } \\
\text { No. }\end{array}$} & \multicolumn{2}{|c|}{$\begin{array}{l}\text { Operating Time } \\
\text { Span for Kiln* }\end{array}$} & \multirow[b]{2}{*}{$\begin{array}{l}\text { Coal } \\
\text { Iype }\end{array}$} & \multirow[b]{2}{*}{$\begin{array}{l}\text { Caust ic } \\
\text { Type }\end{array}$} & \multirow[b]{2}{*}{ Caustic:Coal } & \multirow{2}{*}{\multicolumn{2}{|c|}{ Kiln Skin Temps** }} & \multirow[b]{2}{*}{ 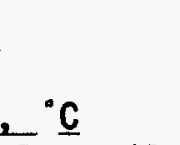 } & \multirow[b]{2}{*}{ Zone \#4 } \\
\hline & $\begin{array}{l}\text { Date } \\
\text { From/To }\end{array}$ & $\begin{array}{l}\text { Time } \\
\text { From/To }\end{array}$ & & & & & & & \\
\hline $5 A 1$ & $\begin{array}{l}3 / 20 \\
3 / 21\end{array}$ & $\begin{array}{l}0100- \\
0055\end{array}$ & $\begin{array}{l}\text { Pittsburgh \#8 } \\
14 \text { Mesh }\end{array}$ & $\begin{array}{l}\mathrm{NaOH}: \mathrm{KOH} \\
(50: 50)\end{array}$ & $2: 1$ & 421 & 410 & 410 & 410 \\
\hline 5B1 & $3 / 21$ & $0055-1900$ & $\begin{array}{l}\text { P1ttsburgh } \# 8 \\
14 \text { Mesh }\end{array}$ & $\mathrm{NaOH}$ & $2: 1$ & 421 & 410 & 410 & 410 \\
\hline 5B2 & $\begin{array}{l}3 / 21 \\
3 / 22\end{array}$ & $\begin{array}{l}1900- \\
0150\end{array}$ & $\begin{array}{l}\text { P1ttsburgh } \# 8 \\
14 \text { Mesh }\end{array}$ & $\mathrm{NaOH}$ & $2: 1$ & 449 & 410 & 410 & 410 \\
\hline 583 & $3 / 22$ & $0150-1215$ & $\begin{array}{l}\text { Pittsburgh } \# 8 \\
14 \text { Mesh }\end{array}$ & $\mathrm{NaOH}$ & $2: 1$ & $\begin{array}{l}\text { Heater } \\
\text { off }\end{array}$ & 449 & 410 & 410 \\
\hline 5B4 & $3 / 22$ & $1215-1600$ & $\begin{array}{l}\text { Pittsburgh } 18 \\
14 \text { Mesh }\end{array}$ & $\mathrm{NaOH}$ & $2: 1$ & $\begin{array}{l}\text { Heater } \\
\text { Off }\end{array}$ & $\begin{array}{l}\text { Heater } \\
\text { Off }\end{array}$ & 449 & 410 \\
\hline $5 B 5$ & $\begin{array}{l}3 / 22 \\
3 / 23\end{array}$ & $\begin{array}{l}1600- \\
0230\end{array}$ & $\begin{array}{l}\text { Ptttsburgh } \# 8 \\
14 \text { Mesh }\end{array}$ & $\mathrm{NaOH}$ & $2: 1$ & 427 & 427 & 427 & 427 \\
\hline $5 C 5$ & $3 / 23$ & $0230-1005$ & $\begin{array}{l}\text { Kentucky "9 } \\
14 \text { Mesh }\end{array}$ & $\mathrm{NaOH}$ & $2: 1$ & 427 & 427 & 427 & 427 \\
\hline $5 \mathrm{C} 4$ & $3 / 23$ & $1005-1800$ & $\begin{array}{l}\text { Kentucky } 19 \\
14 \text { Mesh }\end{array}$ & $\mathrm{NaOH}$ & $2: 1$ & $\begin{array}{l}\text { Heater } \\
\text { off }\end{array}$ & 427 & 427 & 427 \\
\hline
\end{tabular}

* It has been calculated that it takes -8 hours for the coal to get thru the kfln and wash trains. Therefore, when relating product coal collected to kiln conditions this 8 hour lag time must be taken into account.

** Established in later runs that $k 11 n$ internal equilibrium process temperature $-20-25^{\circ} \mathrm{C} 10$ wer than external kiln wall skin temperature. 


\section{Run Condition Summary}

Operating Run No. $5,3 / 20-3 / 24 / 89$

(Cont inued)

\begin{tabular}{|c|c|c|c|c|c|c|c|c|c|}
\hline \multirow[b]{2}{*}{$\begin{array}{l}\text { Run } \\
\text { No. }\end{array}$} & \multicolumn{2}{|c|}{$\begin{array}{l}\text { Operating Time } \\
\text { Span for } k+1 n^{*}\end{array}$} & \multirow[b]{2}{*}{$\begin{array}{l}\text { Coal } \\
\text { Iype }\end{array}$} & \multirow[b]{2}{*}{$\begin{array}{l}\text { Caustic } \\
\text { Type } \\
\end{array}$} & \multirow[b]{2}{*}{ Caust Ic:Coal } & \multirow{2}{*}{\multicolumn{2}{|c|}{ Kłln Skin Temps** }} & \multirow[b]{2}{*}{$={ }^{\circ} \underline{C}$} & \multirow[b]{2}{*}{ Zone \#4 } \\
\hline & $\begin{array}{l}\text { Date } \\
\text { From/To }\end{array}$ & $\begin{array}{l}\text { Time } \\
\text { From/To }\end{array}$ & & & & & & & \\
\hline 501 & $\begin{array}{l}3 / 23 \\
3 / 24\end{array}$ & $\begin{array}{l}1800- \\
0030\end{array}$ & $\begin{array}{l}\text { Kentucky } \# 9 \\
14 \text { Mesh }\end{array}$ & $\mathrm{NaOH}$ & $2: 1$ & $\begin{array}{l}\text { Heater } \\
\text { off }\end{array}$ & $\begin{array}{l}\text { Heater } \\
\text { Off }\end{array}$ & 427 & 427 \\
\hline 502 & $3 / 24$ & $0030-0630$ & $\begin{array}{l}\text { Kentucky } 19 \\
14 \text { Mesh }\end{array}$ & $\mathrm{NaOH}$ & $2: 1$ & 399 & 399 & 399 & 399 \\
\hline 503 & $3 / 24$ & $0630-1220$ & $\begin{array}{l}\text { Kentucky :9 } \\
14 \text { Mesh }\end{array}$ & $\mathrm{NaOH}$ & $2: 1$ & 371 & 371 & 371 & 371 \\
\hline
\end{tabular}

* It has been calculated that it takes -8 hours for the coal to get thru the kiln and wash trains. Therefore, when relating product coal collected to kiln conditions this 8 hour lag time must be taken into account.

* Established in later runs that kfln internal equilibrium process temperature $-20-25^{\circ} \mathrm{C}$ lower than external kiln wall skin temperature. 
MCL OPERATING DATA

RUN NUMBER 6: APR 03-07, 1989

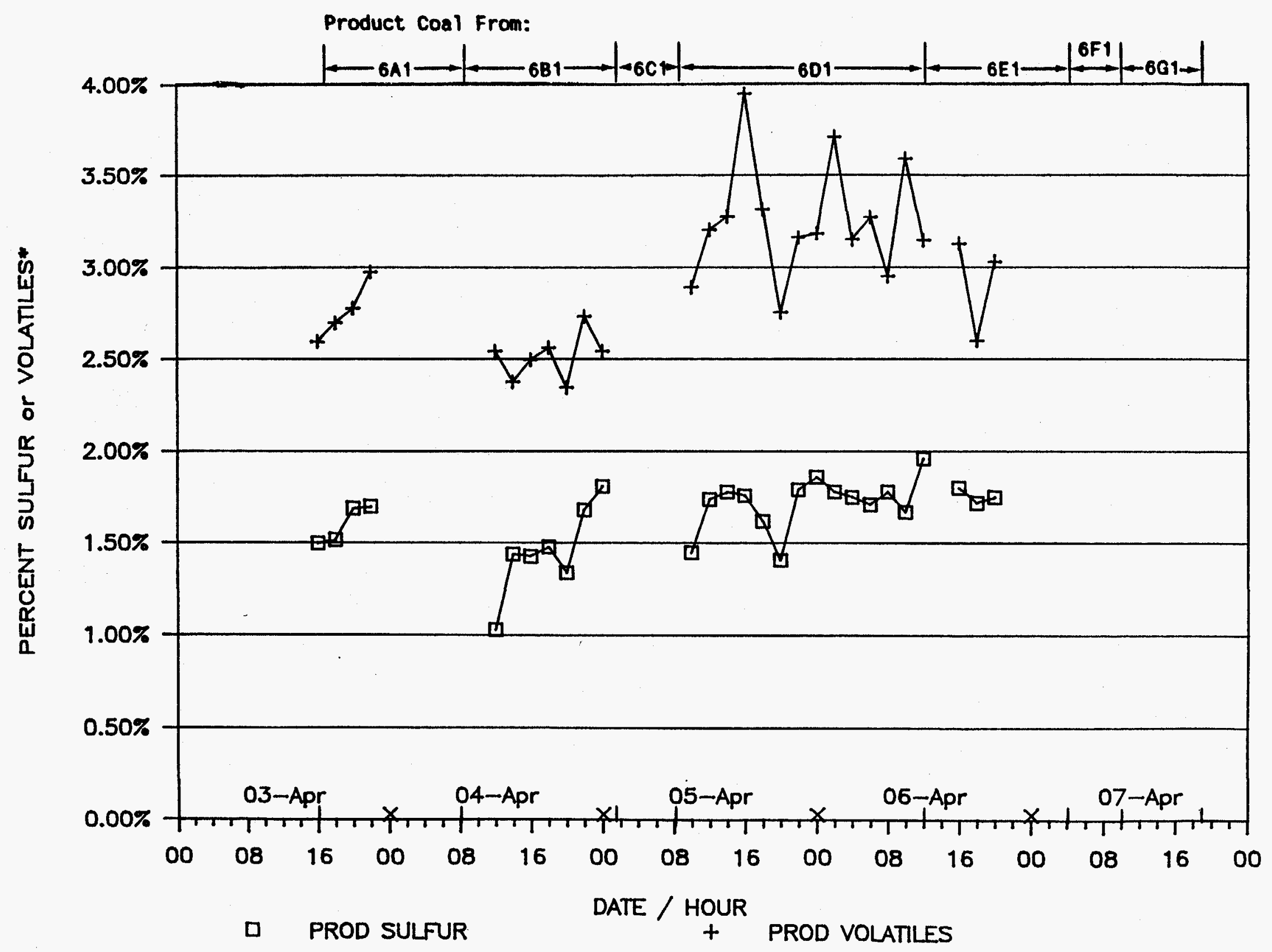

- Volatiles rosding must be eultiplied by a factor of 10. 


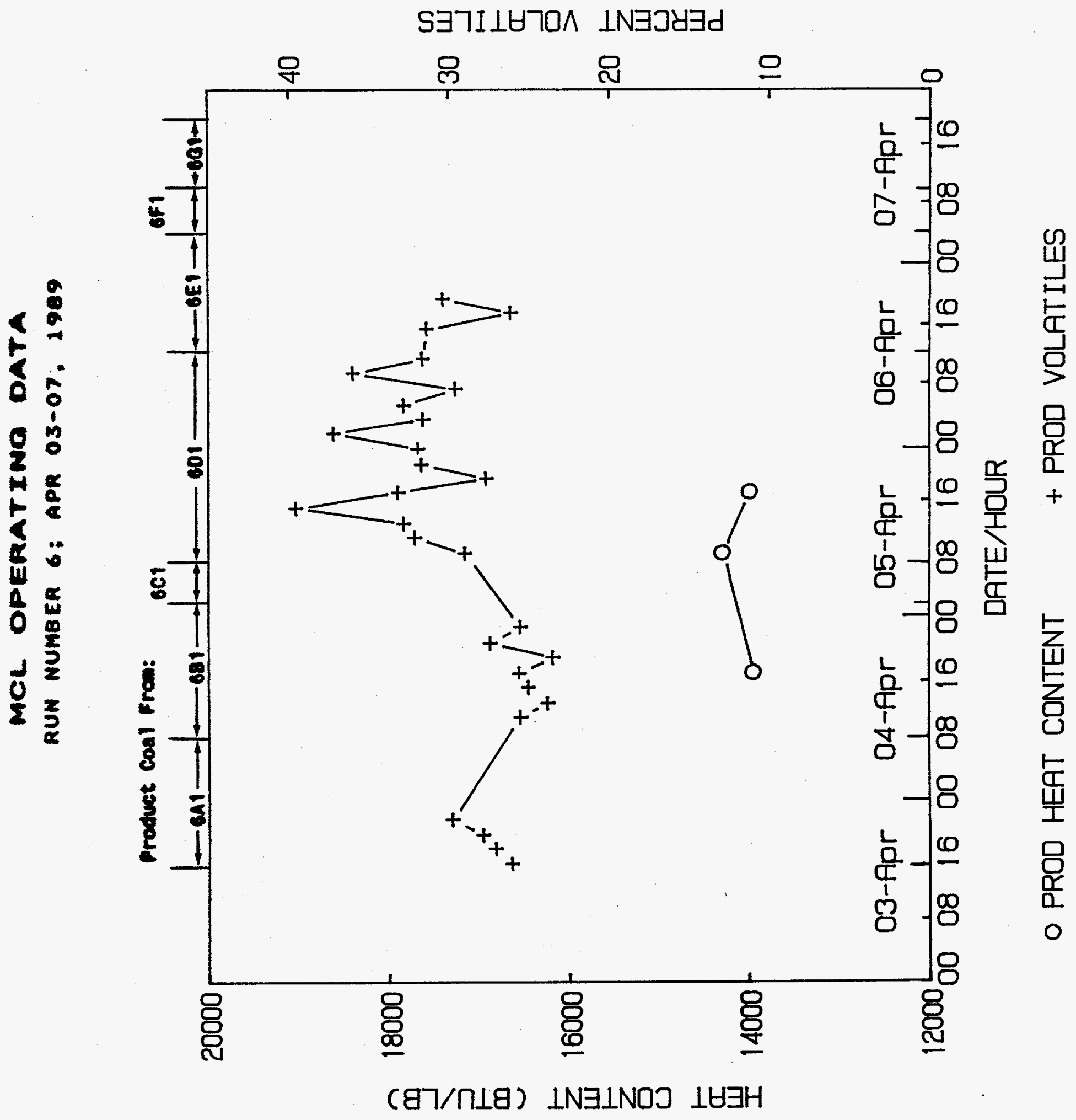




\section{Run Conditton Summary}

Operating Run No. 6, 4/03-4/07/89

Operating Time

Span for Kiln*

Run

Time

Coa 1

Caustic

Type

Caustic:Coal

$\mathrm{Kiln}$ Feed

Kiln Skin

No. From/To From/To Type

$\mathrm{NaOH}$

$1: 1$

Rate $1 \mathrm{bs} / \mathrm{hr}$

Temps " $\mathrm{C}^{* *}$

$6 A 1$

4/03 0400 - Pittsbur

14 Mesh

681

$4 / 04$

$0130-1645$

Pittsburgh $\# 8 \quad \mathrm{NaOH}$

$1.5: 1$

30

A11 zones $427^{\circ} \mathrm{C}$

$6 \mathrm{C1}$

4/04 1645-2130 P1ttsburgh \#8 14 Mesh

$\mathrm{NaOH}$

$1: 1$

30

A11 zones

$427^{\circ} \mathrm{C}$

601

$4 / 05$
$4 / 06$

0000 - P1ttsburgh \#8

14 Mesh

$\mathrm{NaOH}$

$1.5: 1$

$410^{\circ} \mathrm{C}$

on

6E1

$4 / 06 \quad 0515-1900$

P1ttsburgh *8

$\mathrm{NaOH}: \mathrm{KOH}$

14 Mesh

(50:50)

$1: 1$

30

A11 zones $427^{\circ} \mathrm{C}$

6F1

4/06 $1900-$ Pittsburgh 18

$\mathrm{NaOH}: \mathrm{KOH}$

(50:50)

$1: 1$

50

All zones

$427^{\circ} \mathrm{C}$

$6 \mathrm{G1}$

$\begin{array}{lll}4 / 070200-1130 & \begin{array}{l}\text { P1ttsburgh } \# 8 \\ 14 \text { Mesh }\end{array} & \begin{array}{l}\mathrm{NaOH}: \mathrm{KOH} \\ (50: 50)\end{array}\end{array}$

$1: 1$

40

A11 zones $427^{\circ} \mathrm{C}$

* It has been calculated that it takes -8 hours for the coal to get thru the kiln and wash trains. Therefore, when relating product coal collected to kiln conditions this 8 hour lag time must be taken into account.

** Established in later runs that kiln internal equilibrium process temperature $-20-25^{\circ} \mathrm{C} 10 \mathrm{wer}$ than external kiln wall skin temperature. 


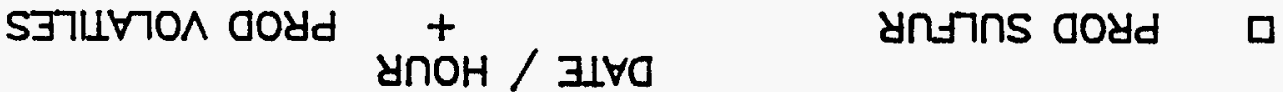

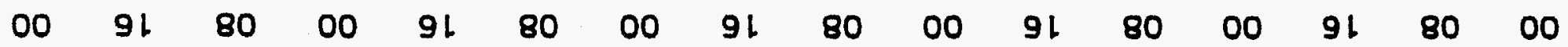

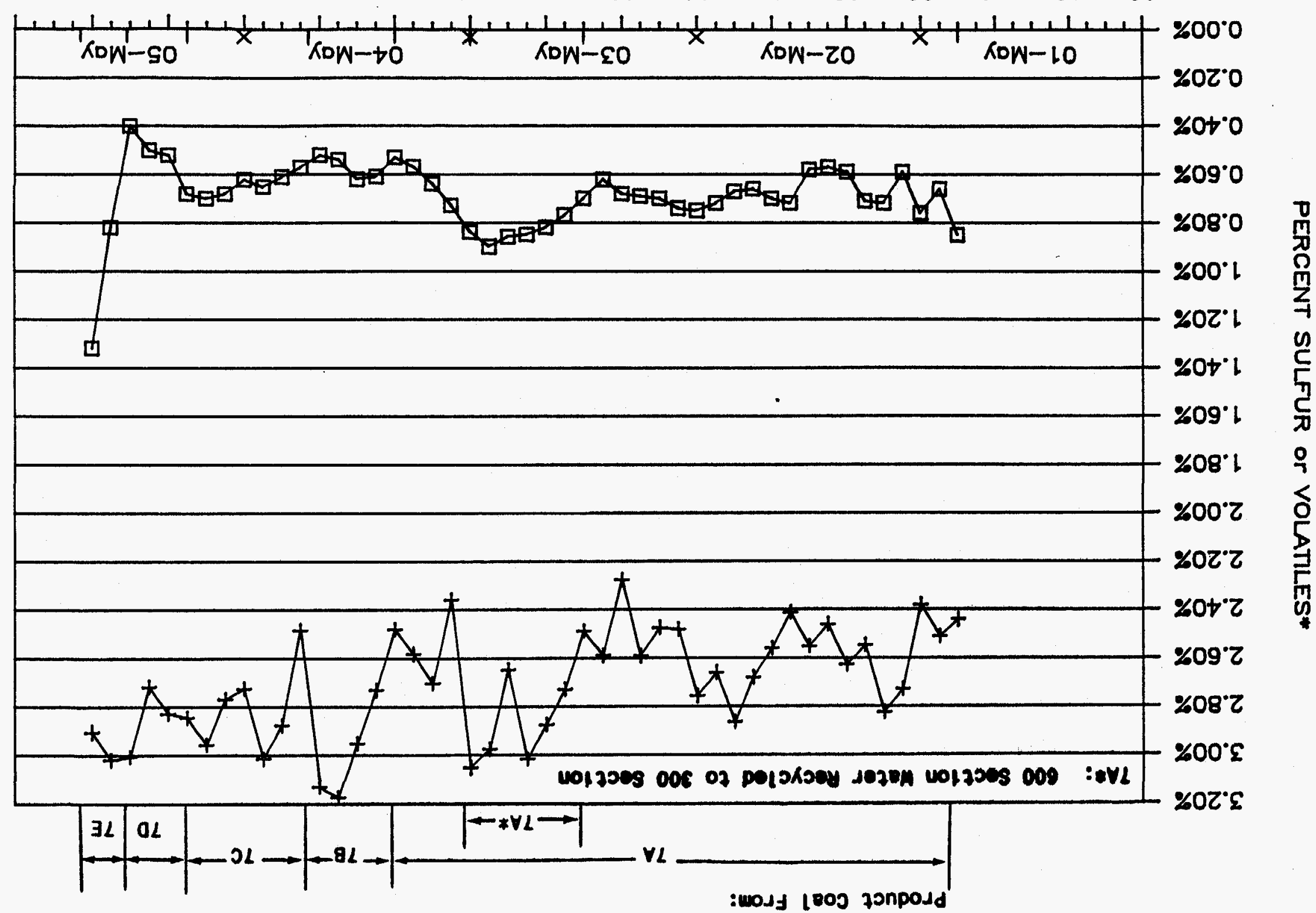




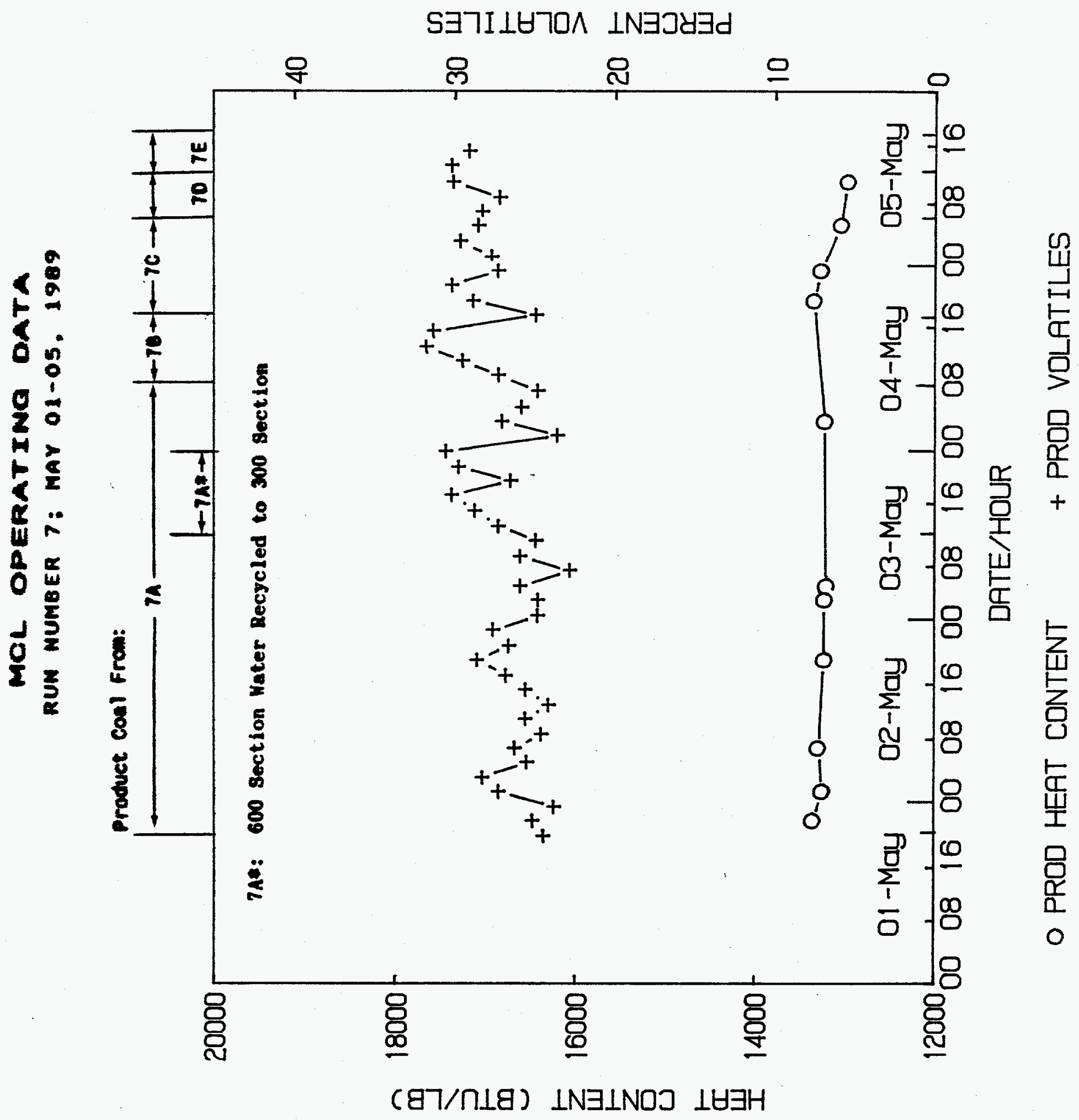


Run Conditton Summary

Operating Run No. 7, 5/01-5/05/89

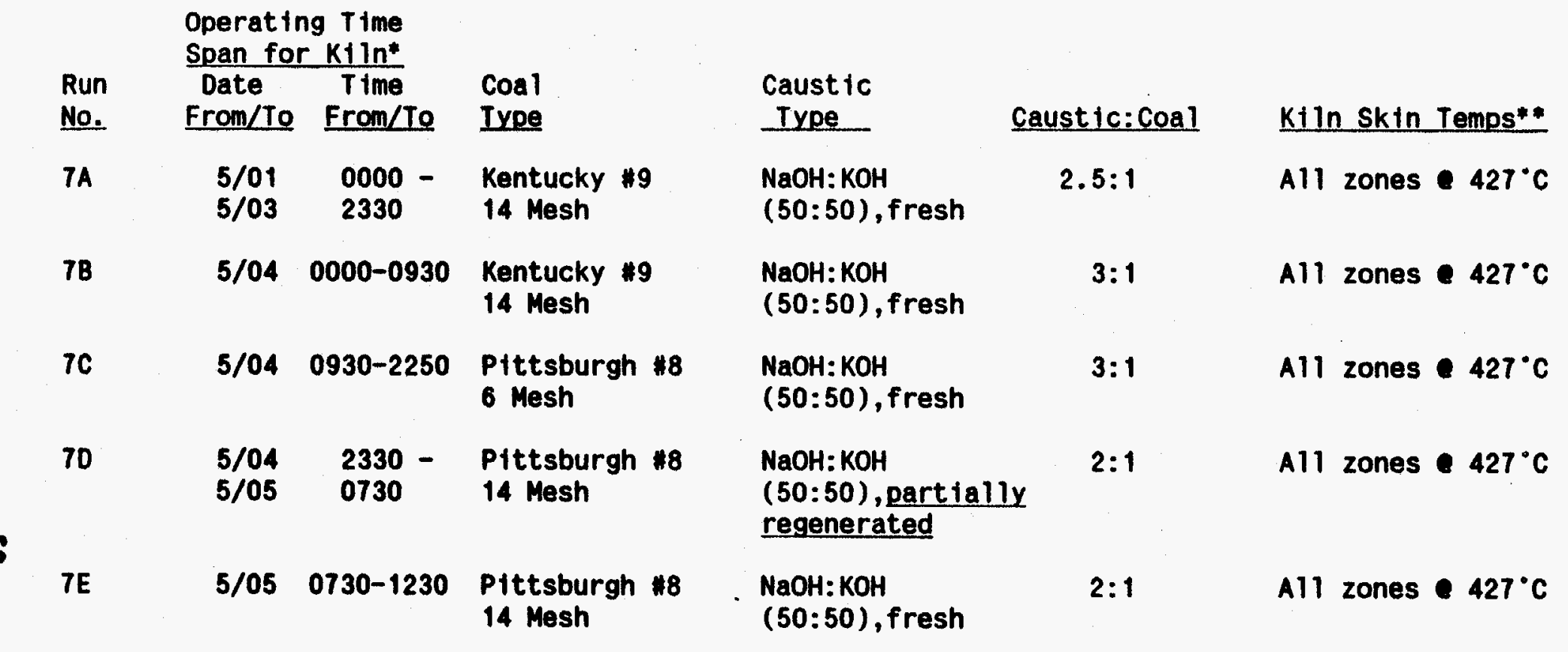

* It has been calculated that it takes -8 hours for the coal to get thru the $k+1 n$ and wash trains. Therefore, when relating product coal collected to ktln conditions this 8 hour lag time must be taken into account.

** Internally measured temperature $-20-25^{\circ} \mathrm{C}$ lower. 
MCL OPERATING DATA

RUN MUMBER B; MAY 15-19, 1989

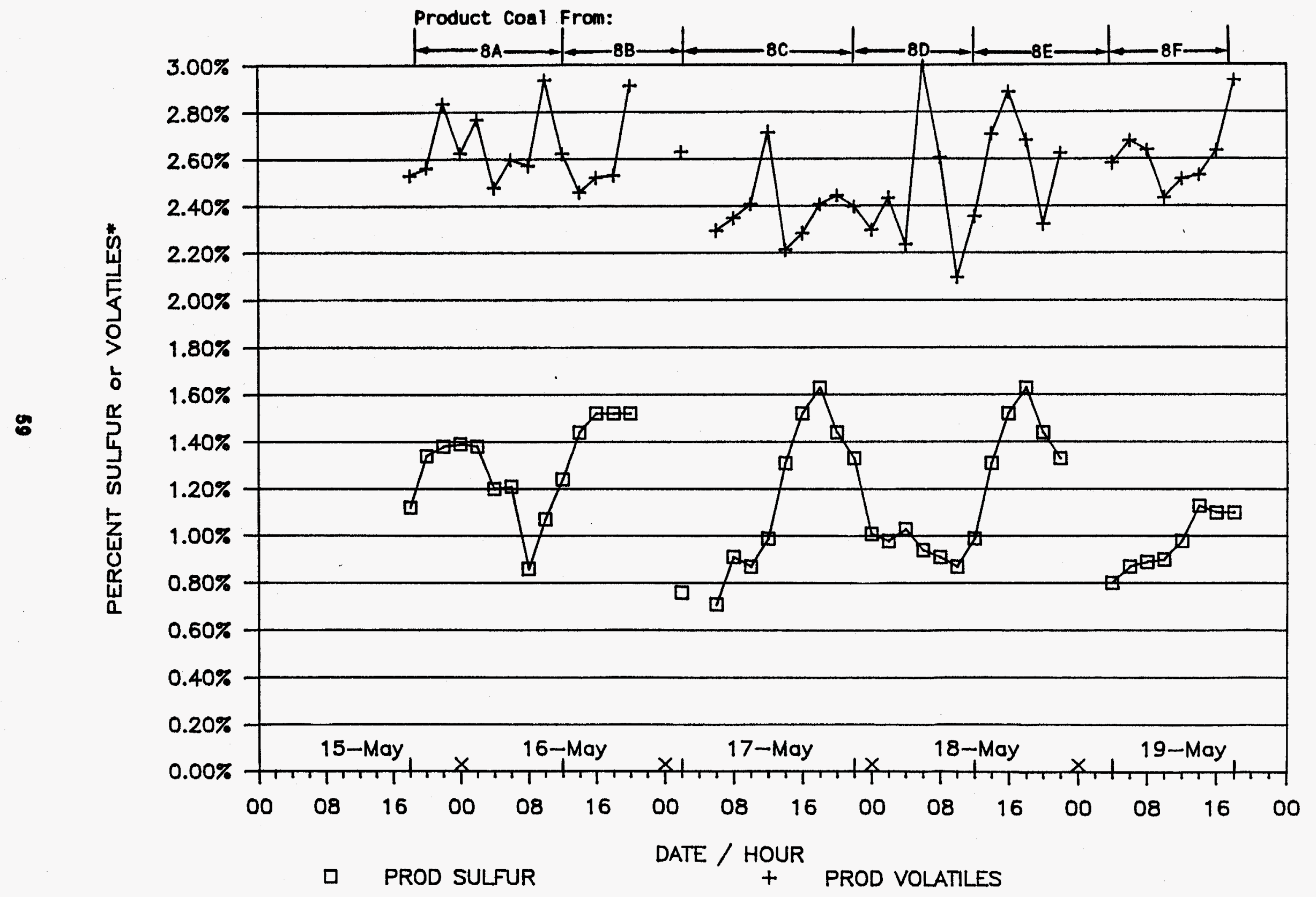

- Volatiles rocdins sust be multiplied by actor of 10 . 

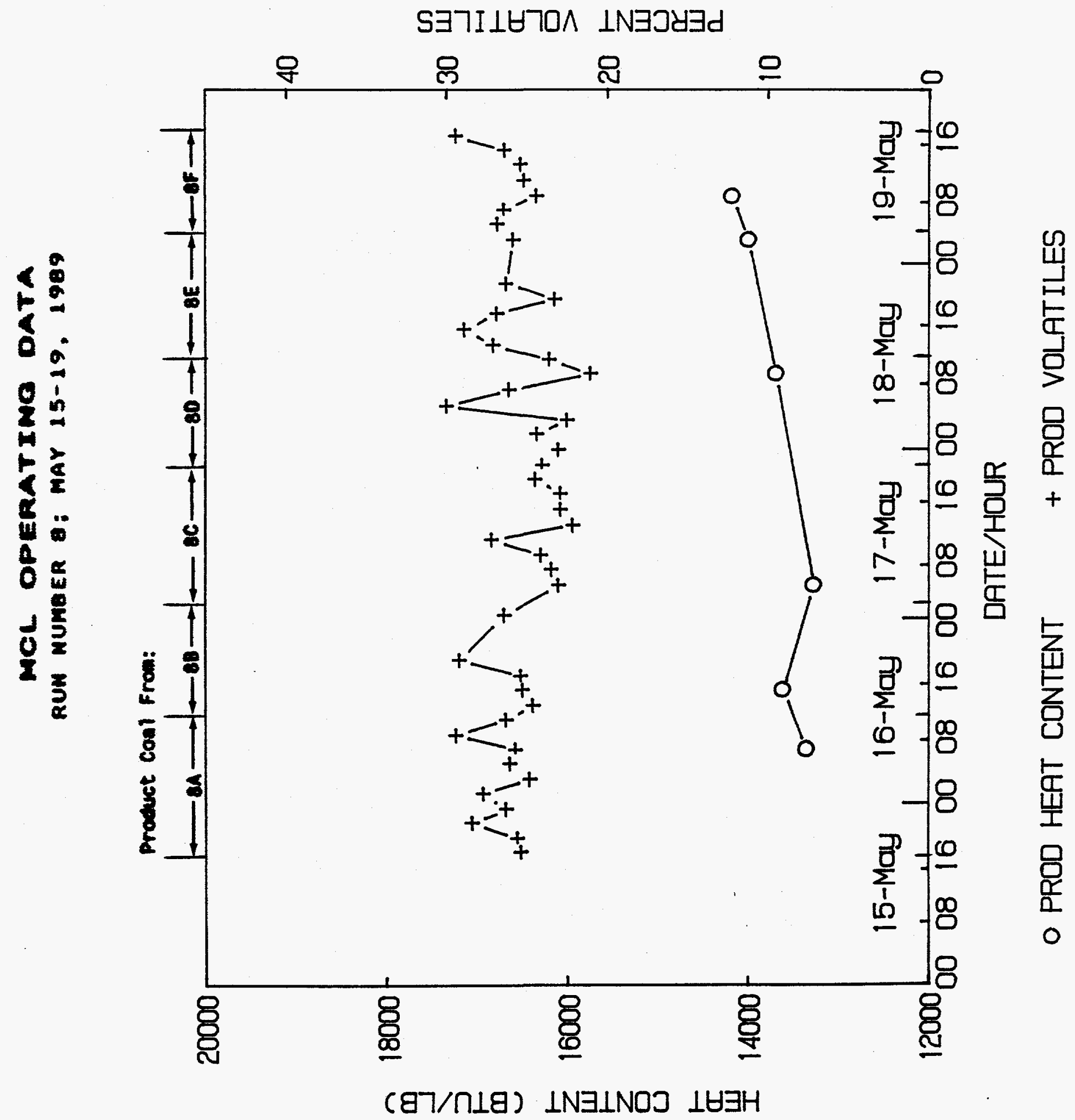
Run Condition Summary

Operating Run No. $8,5 / 15-5 / 19 / 89$

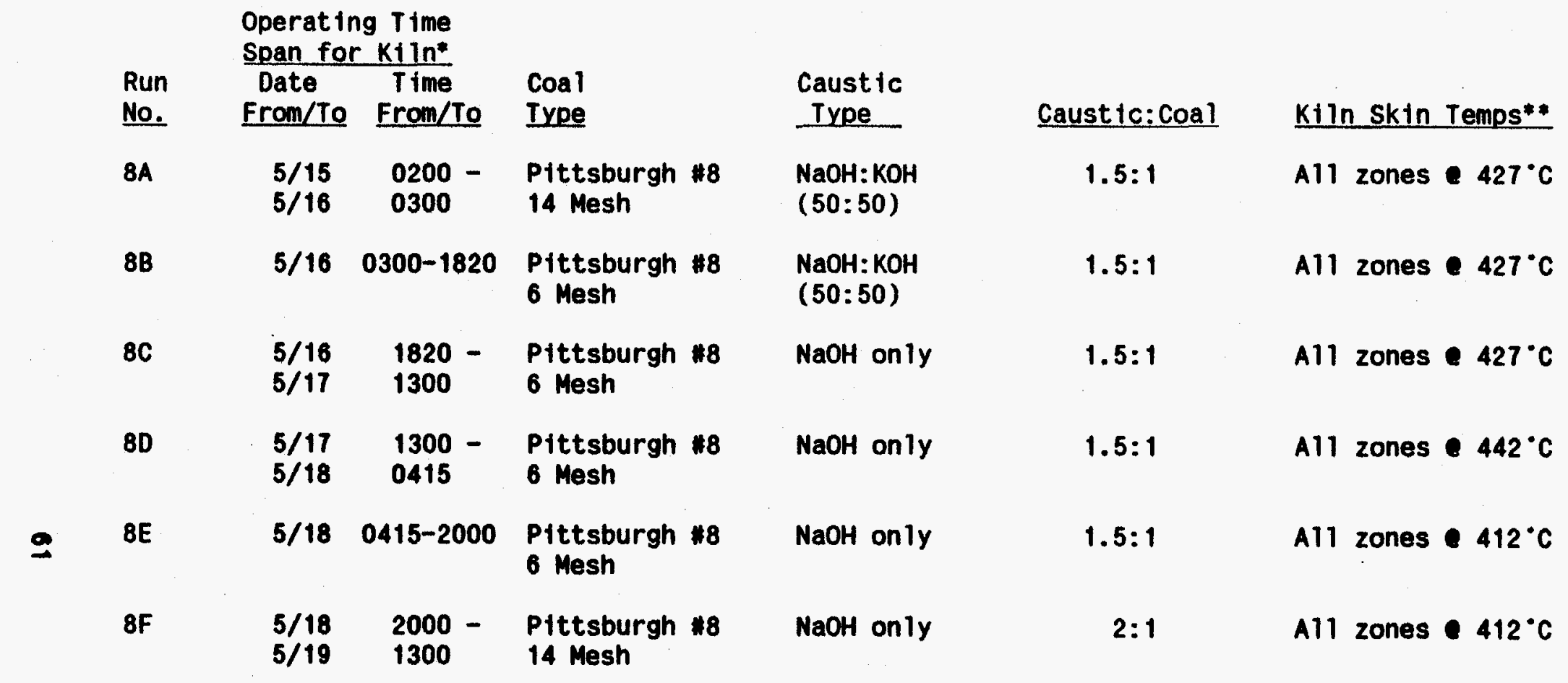

* It has been calculated that it takes -8 hours for the coal to get thru the kiln and wash trains. Therefore, when relating product coal collected to kiln conditions this 8 hour lag time must be taken into account.

** Internally measured temperature $-20-25^{\circ} \mathrm{C}$ lower. 
MCL OPERATING DATA

RUN NUMBER 9; MAY 29-JUNE 02, 1989

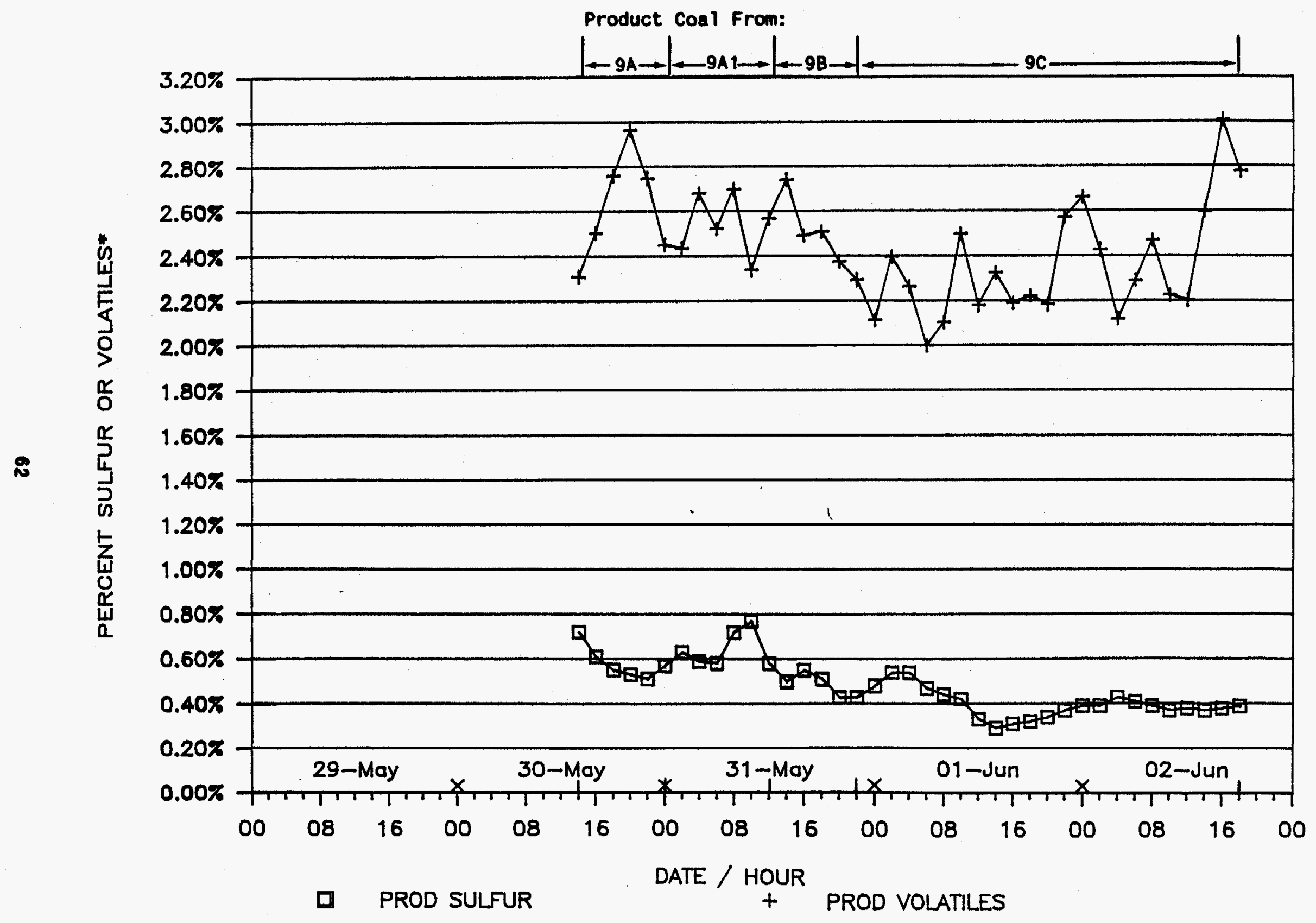

- Volatiles reading must be enitiplited of a fector of 10. 
Run Condttion Summary

Operating Run No, 9, 5/30-6/2/89

\begin{tabular}{|c|c|c|c|c|c|c|}
\hline \multirow[b]{2}{*}{$\begin{array}{l}\text { Run } \\
\text { No. }\end{array}$} & \multicolumn{2}{|c|}{$\begin{array}{l}\text { Operating Time } \\
\text { Span for } k+1 n^{*}\end{array}$} & \multirow[b]{2}{*}{$\begin{array}{l}\text { Coal } \\
\text { Type }\end{array}$} & \multirow[b]{2}{*}{$\begin{array}{l}\text { Caustic } \\
\text { Type } \\
\end{array}$} & \multirow[b]{2}{*}{ Caust 1c:Coal } & \multirow[b]{2}{*}{ Kiln Skin Temps** } \\
\hline & $\begin{array}{l}\text { Date } \\
\text { From/To }\end{array}$ & $\begin{array}{c}\text { Time } \\
\text { Erom/To }\end{array}$ & & & & \\
\hline $9 A$ & $5 / 30$ & $0230-1730$ & $\begin{array}{l}\text { Plttsburgh } \# 8 \\
14 \text { Mesh }\end{array}$ & $\begin{array}{l}\mathrm{NaOH}: \mathrm{KOH} \\
(50: 50), \text { fresh }\end{array}$ & $2.5: 1$ & A11 zones $427^{\circ} \mathrm{C}$ \\
\hline $9 A 1$ & $\begin{array}{l}5 / 30 \\
5 / 31\end{array}$ & $\begin{array}{l}1730- \\
0430\end{array}$ & $\begin{array}{l}\text { Pittsburgh } 18 \\
14 \text { Mesh }\end{array}$ & $\begin{array}{l}\mathrm{NaOH}: \mathrm{KOH} \\
(50: 50) \text {, fresh }\end{array}$ & $2.5: 1$ & A11 zones $438^{\circ} \mathrm{C}$ \\
\hline 9B & $5 / 31$ & $0530-1115$ & $\begin{array}{l}\text { Pittsburgh } \# 8 \\
14 \text { Mesh }\end{array}$ & $\begin{array}{l}\mathrm{NaOH}: \mathrm{KOH} \\
(50: 50), \\
\text { regenerated }\end{array}$ & $2.5: 1$ & A11 zones $438^{\circ} \mathrm{C}$ \\
\hline $9 \mathrm{C}$ & $\begin{array}{l}5 / 31 \\
6 / 02\end{array}$ & $\begin{array}{l}1315- \\
1530\end{array}$ & $\begin{array}{l}\text { Pittsburgh \#8 } \\
14 \text { Mesh }\end{array}$ & $\mathrm{NaOH}$ only, fresh & $2.5: 1$ & All zones $438^{\circ} \mathrm{C}$ \\
\hline
\end{tabular}

ఉ

* It has been calculated that it takes -8 hours for the coal to get thru the $k f l n$ and wash trains. Therefore, when relating product coal collected to $k+1$ n conditions this 8 hour lag time must be taken into account.

** Internally measured temperature $-20-25^{\circ} \mathrm{C}$ lower. 


\subsubsection{Tabulation of Product Coal Analysis Data}


Quantities and Characteristics of Coal Products Collected from MCL Operations

\begin{tabular}{|c|c|c|c|c|c|c|c|c|}
\hline Run & $\begin{array}{l}\text { Coal } \\
\text { Type }\end{array}$ & $\begin{array}{l}\text { Coal } \\
\text { Product } \\
\text { As } \\
\text { Collected }\end{array}$ & $\begin{array}{l}\text { Dry } \\
\text { Coal } \\
\text { Product }\end{array}$ & $\begin{array}{l}\text { \% Sulfur } \\
\text { (Dry } \\
\text { Basis) }\end{array}$ & $\begin{array}{l}\% \text { Ash } \\
\text { (Dry } \\
\text { Basis) }\end{array}$ & $\begin{array}{l}\% \text { Vol } \\
\text { (Dry } \\
\text { Basis) }\end{array}$ & $\begin{array}{l}\text { Btu/1b } \\
\text { (Dry } \\
\text { Basis) }\end{array}$ & $\begin{array}{c}\% \\
\text { Moisture }\end{array}$ \\
\hline $\begin{array}{l}\text { Shake- } \\
\text { jown } \\
\text { vo. } 2\end{array}$ & Pitt 8 & $2411 \mathrm{~b}$ & $1867 \mathrm{~b}$ & $2.5^{\star}$ & $2.8^{*}$ & $40 *$ & $13000 *$ & $23^{\star}$ \\
\hline $\begin{array}{l}A \\
B \\
C \\
D \\
E\end{array}$ & $\begin{array}{l}\text { Pitt } 8 \\
\text { Pitt } 8 \\
\text { Pitt } 8 \\
\text { Pitt } 8 \\
\text { Pitt } 8\end{array}$ & $\begin{array}{rr}62 & 1 \mathrm{~b} \\
192 & 1 \mathrm{~b} \\
125 & 1 \mathrm{~b} \\
171 & 1 \mathrm{~b} \\
42 & 1 \mathrm{~b} \\
592 & 1 \mathrm{~b}\end{array}$ & $\begin{array}{c}301 \mathrm{lb} \\
931 \mathrm{lb} \\
561 \mathrm{lb} \\
77 \mathrm{lb} \\
20 \quad 1 \mathrm{~b} \\
276 \quad 1 \mathrm{~b}\end{array}$ & $\begin{array}{l}1.93^{\star} \\
1.93^{2} \\
1.43 \\
1.39 \\
1.93^{\star}\end{array}$ & $\begin{array}{l}2.32 \\
2.08 \\
1.32 \\
1.12 \\
2.43^{\star}\end{array}$ & $\begin{array}{l}32.77 \\
34.88 \\
27.40 \\
30.36 \\
30.68 *\end{array}$ & $\begin{array}{l}13000^{*} \\
13000^{*} \\
13000^{*} \\
13000^{*} \\
13000^{*}\end{array}$ & $\begin{array}{l}51.59^{\star} \\
51.59 \\
55.10 \\
55.17 \\
51.59^{\star}\end{array}$ \\
\hline $\begin{array}{l}\text { ?B } \\
\text { ?C } \\
\text { ?D } \\
\text { ?E }\end{array}$ & $\begin{array}{l}\text { Pitt } 8 \\
\text { Pitt } 8 \\
\text { Kent } 9 \\
\text { Kent } 9\end{array}$ & $\begin{array}{rr}84 & 1 \mathrm{~b} \\
216 & 1 \mathrm{~b} \\
189 & 1 \mathrm{~b} \\
192 & 1 \mathrm{~b} \\
681 & 1 \mathrm{~b}\end{array}$ & $\begin{array}{rr}44 & 1 b \\
89 & 1 b \\
84 & 1 b \\
89 & 1 b \\
306 & 1 b\end{array}$ & $\begin{array}{l}1.93^{\star} \\
0.77 \\
0.78 \\
0.89\end{array}$ & $\begin{array}{l}2.43 \\
0.64 \\
0.47 \\
0.43\end{array}$ & $\begin{array}{l}30.68 \\
28.37 \\
29.94 \\
30.27\end{array}$ & $\begin{array}{l}13000^{*} \\
13092 \\
13226 \\
13000^{\star}\end{array}$ & $\begin{array}{l}47.03 \\
58.83 \\
55.72 \\
53.89\end{array}$ \\
\hline $\begin{array}{l}3 B 1 \\
3 C 1\end{array}$ & $\begin{array}{l}\text { Pitt } 8 \\
\text { Pitt } 8\end{array}$ & $\begin{array}{r}14416 \\
3216 \\
176 \quad 16\end{array}$ & $\begin{array}{r}641 b \\
141 b \\
7816\end{array}$ & $\begin{array}{l}0.71 \\
0.72\end{array}$ & $\begin{array}{l}0.23 \\
0.23\end{array}$ & $\begin{array}{l}20.84 \\
20.93\end{array}$ & $\begin{array}{l}12617 \\
12617^{\star}\end{array}$ & $\begin{array}{l}55.74 \\
56.35\end{array}$ \\
\hline 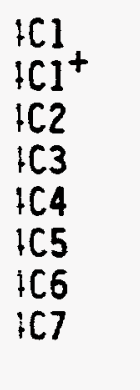 & $\begin{array}{l}\text { Pitt } 8 \\
\text { Pitt } 8 \\
\text { Pitt } 8 \\
\text { Pitt } 8 \\
\text { Pitt } 8 \\
\text { Pitt } 8 \\
\text { Pitt } 8 \\
\text { Pitt } 8\end{array}$ & $\begin{array}{rl}151 & 1 \mathrm{~b} \\
19 & 1 \mathrm{~b} \\
154 & 1 \mathrm{~b} \\
85 & 1 \mathrm{~b} \\
65 & 1 \mathrm{~b} \\
58 & 1 \mathrm{~b} \\
76 & 1 \mathrm{~b} \\
69 & 1 \mathrm{~b} \\
658 & 1 \mathrm{~b}\end{array}$ & $\begin{array}{rr}72 & 1 b \\
9 & 1 b \\
73 & 1 b \\
40 & 1 b \\
31 & 1 b \\
27 & 1 b \\
37 & 1 b \\
33 & 1 b \\
313 & 1 b\end{array}$ & $\begin{array}{l}1.14 \\
0.77 \\
1.25 \\
1.22 \\
1.17 \\
1.35 \\
1.42 \\
1.22\end{array}$ & $\begin{array}{l}0.68 \\
0.64 \\
0.54 \\
0.52 \\
0.46 \\
0.68 \\
0.72 \\
0.64\end{array}$ & $\begin{array}{l}24.65 \\
23.73 \\
23.91 \\
22.36 \\
24.59 \\
27.22 \\
27.65 \\
26.05\end{array}$ & $\begin{array}{l}14226 \\
14241 \\
14219 \\
14000^{*} \\
14201 \\
14000^{\star} \\
14268 \\
14291\end{array}$ & $\begin{array}{l}52.45 \\
54.16 \\
52.38 \\
52.52 \\
52.89 \\
53.14 \\
51.31 \\
52.45\end{array}$ \\
\hline
\end{tabular}

\footnotetext{
*Estimated values

Quantities of coal products collected are subject to confirmation

$4 \mathrm{Cl}^{+}$represents coal product collected at $13: 45,16: 00,17: 10$ and $18: 10$ on 3/7/1989, or the first hours of Run $4 \mathrm{Cl}$. Compliance coal was produced during this period.
} 
Quantities and Characteristics of Coal Products Collected from MCL Operations (Continued)

\begin{tabular}{|c|c|c|c|c|c|c|c|c|}
\hline Zun & $\begin{array}{l}\text { Coal } \\
\text { Type }\end{array}$ & $\begin{array}{l}\text { Coal } \\
\text { Product } \\
\text { As } \\
\text { Collected }\end{array}$ & $\begin{array}{l}\text { Dry } \\
\text { Coal } \\
\text { Product }\end{array}$ & $\begin{array}{l}\text { \% Sulfur } \\
\text { (Dry } \\
\text { Basis) }\end{array}$ & $\begin{array}{l}\text { \% Ash } \\
\text { (Dry } \\
\text { Basis) }\end{array}$ & $\begin{array}{l}\text { \% Vol } \\
\text { (Dry } \\
\text { Basis) }\end{array}$ & $\begin{array}{l}\text { Btu/lb } \\
\text { (Dry } \\
\text { Basis) }\end{array}$ & $\begin{array}{c}\% \\
\text { Moisture }\end{array}$ \\
\hline $\begin{array}{l}j A 1 \\
j B 1 \\
j B 2 \\
j B 3 \\
j B 4 \\
j B 5 \\
j C 5 \\
j C 4 \\
j D 1 \\
j D 2\end{array}$ & $\begin{array}{l}\text { Pitt } 8 \\
\text { Pitt } 8 \\
\text { Pitt } 8 \\
\text { Pitt } 8 \\
\text { Pitt } 8 \\
\text { Pitt } 8 \\
\text { Kent } 9 \\
\text { Kent } 9 \\
\text { Kent } 9 \\
\text { Kent } 9\end{array}$ & 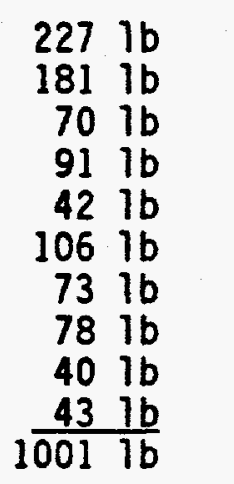 & $\begin{array}{rl}124 & \mathrm{lb} \\
81 & \mathrm{ib} \\
30 & \mathrm{ib} \\
41 & \mathrm{lb} \\
19 & \mathrm{ib} \\
48 & \mathrm{ib} \\
35 & \mathrm{ib} \\
35 & \mathrm{ib} \\
19 & \mathrm{ib} \\
21 & 1 \mathrm{~b} \\
453 & 1 \mathrm{~b}\end{array}$ & $\begin{array}{l}1.31 \\
1.32 \\
1.20 \\
1.46 \\
1.60 \\
1.19 \\
1.10 \\
1.11 \\
1.46 \\
1.27\end{array}$ & $\begin{array}{l}1.23 \\
0.67 \\
0.58 \\
0.63 \\
0.95 \\
0.45 \\
0.37 \\
0.50 \\
1.03 \\
0.80\end{array}$ & $\begin{array}{l}24.52 \\
24.47 \\
24.65 \\
24.69 \\
26.39 \\
21.49 \\
22.57 \\
23.66 \\
29.48 \\
27.64\end{array}$ & $\begin{array}{l}13686 \\
14052 \\
14059 \\
14023 \\
14047 \\
13656 \\
13651 \\
13918 \\
14130 \\
14152\end{array}$ & $\begin{array}{l}55.28 \\
55.16 \\
56.61 \\
54.40 \\
55.23 \\
54.81 \\
51.69 \\
55.73 \\
53.65 \\
52.05\end{array}$ \\
\hline $\begin{array}{l}j A 1 \\
j B 1 \\
j C 1 \\
j D 1 \\
j E 1\end{array}$ & $\begin{array}{l}\text { Pitt } 8 \\
\text { Pitt } 8 \\
\text { Pitt } 8 \\
\text { Pitt } 8 \\
\text { Pitt } 8\end{array}$ & $\begin{array}{r}91 \mathrm{lb} \\
160 \mathrm{lb} \\
11 \mathrm{lb} \\
419 \mathrm{lb} \\
1401 \mathrm{~b} \\
821 \mathrm{1b}\end{array}$ & $\begin{array}{r}43 \mathrm{lb} \\
74 \mathrm{ib} \\
5 \mathrm{lb} \\
190 \mathrm{ib} \\
73 \mathrm{lb} \\
385 \mathrm{ib}\end{array}$ & $\begin{array}{l}1.60 \\
1.55 \\
1.74^{*} \\
1.74 \\
1.72\end{array}$ & $\begin{array}{l}1.14 \\
0.64 \\
1.14^{\star} \\
0.87 \\
2.02\end{array}$ & $\begin{array}{l}27.65 \\
25.15 \\
32.56^{\star} \\
32.56 \\
30.84\end{array}$ & $\begin{array}{l}13948^{\star} \\
13948 \\
14000^{\star} \\
14150 \\
13000^{\star}\end{array}$ & $\begin{array}{l}52.24 \\
53.93 \\
52.24 \\
54.74 \\
47.73\end{array}$ \\
\hline $\begin{array}{l}\text { TA } \\
T A^{+} \\
T B \\
T C \\
' D \\
' D\end{array}$ & $\begin{array}{l}\text { Kent } 9 \\
\text { Kent } 9 \\
\text { Kent } 9 \\
\text { Pitt } 8 \\
\text { Pitt } 8 \\
\text { Pitt } 8\end{array}$ & $\begin{array}{r}571 \mathrm{lb} \\
30 \mathrm{lb} \\
85 \mathrm{lb} \\
80 \mathrm{lb} \\
29 \mathrm{lb} \\
13 \mathrm{lb} \\
778 \mathrm{lb}\end{array}$ & $\begin{array}{r}238 \mathrm{lb} \\
12 \mathrm{lb} \\
35 \mathrm{lb} \\
33 \mathrm{lb} \\
13 \mathrm{lb} \\
6 \mathrm{lb} \\
325 \mathrm{lb}\end{array}$ & $\begin{array}{l}0.71 \\
0.58 \\
0.56 \\
0.64 \\
0.56 \\
1.32\end{array}$ & $\begin{array}{l}0.55 \\
0.38 \\
0.27 \\
0.68 \\
0.58 \\
1.42\end{array}$ & $\begin{array}{l}26.36 \\
25.49 \\
29.07 \\
28.13 \\
28.94 \\
29.15\end{array}$ & $\begin{array}{l}13262 \\
13262 \\
13285 \\
13349 \\
12976 \\
13349 *\end{array}$ & $\begin{array}{l}58.26 \\
59.29 \\
59.37 \\
58.35 \\
55.86 \\
51.35\end{array}$ \\
\hline $\begin{array}{l}3 A \\
3 B \\
3 C \\
3 D \\
3 D \\
3 F\end{array}$ & $\begin{array}{l}\text { Pitt } 8 \\
\text { Pitt } 8 \\
\text { Pitt } 8 \\
\text { Pitt } 8 \\
\text { Pitt } 8 \\
\text { Pitt } 8\end{array}$ & 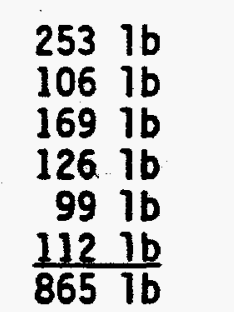 & $\begin{array}{r}113 \mathrm{lb} \\
50 \mathrm{ib} \\
82 \mathrm{ib} \\
60 \mathrm{ib} \\
48 \mathrm{ib} \\
\frac{52 \mathrm{ib}}{405 \mathrm{ib}}\end{array}$ & $\begin{array}{l}1.22 \\
1.35 \\
1.18 \\
0.96 \\
1.46 \\
0.97\end{array}$ & $\begin{array}{l}1.01 \\
1.95 \\
1.20 \\
1.03 \\
1.18 \\
0.54\end{array}$ & $\begin{array}{l}26.54 \\
26.12 \\
23.91 \\
24.41 \\
26.45 \\
26.19\end{array}$ & $\begin{array}{l}13359 \\
13616 \\
13278 \\
13694 \\
14001 \\
14177\end{array}$ & $\begin{array}{l}55.14 \\
52.66 \\
51.58 \\
52.12 \\
51.34 \\
54.03\end{array}$ \\
\hline
\end{tabular}

\footnotetext{
*Estimated values

Quantities of coal products collected are subject to confirmation

$7 A^{+}$represents coal product collected at $8: 15,9: 15,10: 15$ and 1215 on 5/2/1989.

Compliance coal was produced during this period of $7 \mathrm{~A}$.
} 
Quantities and Characteristics of Coal Products Collected from MCL Operations (Continued)

\begin{tabular}{|c|c|c|c|c|c|c|c|c|}
\hline Sun & $\begin{array}{l}\text { Coal } \\
\text { Type }\end{array}$ & $\begin{array}{l}\text { Coal } \\
\text { Product } \\
\text { As } \\
\text { Collected }\end{array}$ & $\begin{array}{l}\text { Dry } \\
\text { Coal } \\
\text { Product }\end{array}$ & $\begin{array}{l}\text { \% Sulfur } \\
\text { (Dry } \\
\text { Basis) }\end{array}$ & $\begin{array}{l}\% \text { Ash } \\
\text { (Dry } \\
\text { Basis) }\end{array}$ & $\begin{array}{l}\% \text { Vol } \\
\text { (Dry } \\
\text { Basis) }\end{array}$ & $\begin{array}{l}\text { Btu/lb } \\
\text { (Dry } \\
\text { Basis) }\end{array}$ & $\begin{array}{c}\% \\
\text { Moisture }\end{array}$ \\
\hline $\begin{array}{l}A A \\
A A] \\
B \\
C\end{array}$ & $\begin{array}{l}\text { Pitt } 8 \\
\text { Pitt } 8 \\
\text { Pitt } 8 \\
\text { Pitt } 8\end{array}$ & $\begin{array}{rl}100 & \mathrm{lb} \\
82 & \mathrm{lb} \\
58 & \mathrm{lb} \\
3457 \mathrm{bb} \\
585 \mathrm{lb}\end{array}$ & $\begin{array}{rr}41 & 1 \mathrm{~b} \\
35 & \mathrm{lb} \\
26 & \mathrm{jb} \\
150 & \mathrm{lb} \\
252 & 1 \mathrm{~b}\end{array}$ & $\begin{array}{l}0.59 \\
0.65 \\
0.49 \\
0.40\end{array}$ & $\begin{array}{l}0.27 \\
0.33 \\
0.24 \\
0.15\end{array}$ & $\begin{array}{l}25.97 \\
25.63 \\
24.84 \\
23.65^{\star \star}\end{array}$ & $\begin{array}{l}13000^{\star} \\
13000^{\star} \\
13000^{\star} \\
14000^{\star}\end{array}$ & $\begin{array}{l}58.83 \\
57.39 \\
56.11 \\
56.49\end{array}$ \\
\hline
\end{tabular}

*Estimated values

Quantities of coal products collected are subject to confirmation

$* *$ Volatile content of product coal approaches $30 \%$ near end of run 


\subsection{REGENERATION OF CAUSTIC SOLUTION}

Although the caustic solution obtained from the current $\mathrm{MCL}$ operation contained less than 15 percent caustic, analysis results of the regenerator inlet (VF2037) and outlet (VF4024) samples indicate significant removal of silicon and aluminum compounds. Regeneration was conducted by the addition of lime to the caustic solution, typically at a temperature of $110^{\circ} \mathrm{F}\left(43^{\circ} \mathrm{C}\right)$. The VF4024 samples were acquired after the the regenerated solution was centrifuged for solids removal, followed by passing the centrate through a bag filter. The spent caustic solution was also passed through a bag filter prior to entering the regenerator. During selected runs, samples (VP2023) of the spent caustic solution were collected upstream of this bag filter. Comparison of the VF2037 and VP2023 data would provide an indication whether some of the mineral matter was removed in the bag filter even before entering the regenerator unit.

The concentration levels of aluminum, silicon and iron present in the VF2037, VF4024 and VP2023 samples are presented in Table 1. Also presented in this table are the computed concentration levels based on the coal product generation rate, assuming that all the mineral matter from coal is in solution. These computed concentration levels can be compared with the concentration levels of aluminum, silicon and iron in the VF2037 and VP2023 samples.

The data presented in Table 1 show that essentially all calculated aluminum and silicon in the coal feed were found in the caustic regenerator feed (VP2023 and VF2037). The expected aluminum and silicon concentrations in this stream are in fairly good agreement with the measured values.

For Runs $3 C 1,7 A, 8 A, 8 E$ and $8 F$, some silicon and aluminum in the caustic regeneration feed was precipitated in the bag filter. The amount precipitated is a function of the residence time in the filter tank prior to pumping to the caustic regenerator, and may also depend on the temperature and $\mathrm{pH}$ of the feed solution. 
Table 1. Concentration Levels of Aluminum, Silicon and Iron in Spent and Regenerated Caust ic

\begin{tabular}{|c|c|c|c|c|}
\hline Run & Sample & A1 $(\mathrm{mg} / \mathrm{L})$ & si $(\mathrm{mg} / \mathrm{L})$ & $\mathrm{Fe}(\mathrm{mg} / \mathrm{L}$ \\
\hline $\begin{array}{l}2 C \\
2 C \\
2 C\end{array}$ & $\begin{array}{l}\text { VF2037 } \\
\text { VF4024 } \\
\text { Computed }\end{array}$ & $\begin{array}{l}540 \\
374 \\
451\end{array}$ & $\begin{array}{r}1120 \\
142 \\
805\end{array}$ & $\begin{array}{r}36 \\
44 \\
787\end{array}$ \\
\hline $\begin{array}{l}3 \mathrm{~B} 1 \\
3 \mathrm{~B} 1 \\
3 \mathrm{~B} 1 \\
3 \mathrm{~B} 1\end{array}$ & $\begin{array}{l}\text { VP2023 } \\
\text { VF2037 } \\
\text { VF4024 } \\
\text { Computed }\end{array}$ & $\begin{array}{l}430 \\
453 \\
353 \\
380\end{array}$ & $\begin{array}{l}950 \\
888 \\
612 \\
678\end{array}$ & $\begin{array}{r}110 \\
105 \\
31 \\
663\end{array}$ \\
\hline $\begin{array}{l}3 \mathrm{CI} \\
3 \mathrm{C1} \\
3 \mathrm{C1} \\
3 \mathrm{C1}\end{array}$ & $\begin{array}{l}\text { VP2023 } \\
\text { VF2037 } \\
\text { VF4024 } \\
\text { Computed }\end{array}$ & $\begin{array}{l}540 \\
515 \\
400 \\
418\end{array}$ & $\begin{array}{r}1300 \\
1170 \\
650 \\
746\end{array}$ & $\begin{array}{r}140 \\
140 \\
30 \\
729\end{array}$ \\
\hline $\begin{array}{l}5 B \\
5 B \\
5 B\end{array}$ & $\begin{array}{l}\text { VF2037 } \\
\text { VF4024 } \\
\text { Computed }\end{array}$ & $\begin{array}{l}482 \\
664 \\
574\end{array}$ & $\begin{array}{r}989 \\
411 \\
1024\end{array}$ & $\begin{array}{r}65 \\
31 \\
1002\end{array}$ \\
\hline $\begin{array}{l}7 A \\
7 A \\
7 A \\
7 A\end{array}$ & $\begin{array}{l}\text { VP2023 } \\
\text { VF2037 } \\
\text { VF4024 } \\
\text { Computed }\end{array}$ & $\begin{array}{l}378 \\
302 \\
352 \\
521\end{array}$ & $\begin{array}{l}864 \\
756 \\
264 \\
783\end{array}$ & $\begin{array}{r}378 \\
30 \\
44 \\
711\end{array}$ \\
\hline $\begin{array}{l}8 A \\
8 A \\
8 A \\
8 A\end{array}$ & $\begin{array}{l}\text { VP2023 } \\
\text { VF2037 } \\
\text { VF4024 } \\
\text { Computed }\end{array}$ & $\begin{array}{l}949 \\
860 \\
659 \\
918\end{array}$ & $\begin{array}{r}2077 \\
1873 \\
395 \\
1637\end{array}$ & $\begin{array}{r}748 \\
483 \\
28 \\
1601\end{array}$ \\
\hline $\begin{array}{l}8 C \\
8 C \\
8 C \\
8 C\end{array}$ & $\begin{array}{l}\text { VP2023 } \\
\text { VF2037 } \\
\text { VF4024 } \\
\text { Computed }\end{array}$ & $\begin{array}{l}489 \\
482 \\
163 \\
518\end{array}$ & $\begin{array}{l}917 \\
959 \\
178 \\
924\end{array}$ & $\begin{array}{r}58 \\
120 \\
5 \\
903\end{array}$ \\
\hline $\begin{array}{l}8 E \\
8 E \\
8 E \\
8 E\end{array}$ & $\begin{array}{l}\text { VP2023 } \\
\text { VF2037 } \\
\text { VF4024 } \\
\text { Computed }\end{array}$ & $\begin{array}{l}311 \\
250 \\
379 \\
427\end{array}$ & $\begin{array}{l}642 \\
531 \\
139 \\
761\end{array}$ & $\begin{array}{r}88 \\
89 \\
11 \\
744\end{array}$ \\
\hline $\begin{array}{l}8 F \\
8 F \\
8 F \\
8 F\end{array}$ & $\begin{array}{l}\text { VP2023 } \\
\text { VF2037 } \\
\text { VF4024 } \\
\text { Computed }\end{array}$ & $\begin{array}{l}774 \\
512 \\
348 \\
542\end{array}$ & $\begin{array}{r}1473 \\
1050 \\
165 \\
966\end{array}$ & $\begin{array}{r}225 \\
152 \\
11 \\
945\end{array}$ \\
\hline
\end{tabular}


For Runs $7 \mathrm{~A}$ and $8 \mathrm{~A}$, iron removed from the coal feed was equally partitioned between the caustic regeneration feed (before filtration) and the acid wash water. However, a significant fraction of the iron that partitioned to the caustic regeneration feed was removed in the bag filter. For the other runs, most of the iron was found in the acid wash water and not in the caustic regeneration feed. The amount of iron in the caustic regeneration feed may depend on the concentration of the caustic solutions.

The aluminum concentration levels in VF2037 were usually higher but sometimes lower than those in the corresponding VF4024 samples. This is because the sample concentration levels represent instantaneous values which fluctuated during the course of the run. The calculated removal efficiency for aluminum ranges from 0 to 66.2 percent and averages 21.9 percent. Removal efficiency for silicon ranges from 31.1 to 87.3 percent and averages 67.2 percent. The iron concentration in the spent caustic solution was typically fairly low, as most of the iron in coal feed is partitioned to the acid wash water. Regardless of the the iron concentration in the spent caustic solution, the regenerated caustic contained 5 to $44 \mathrm{mg} / \mathrm{L}$ iron. In a concentrated caustic solution, and for treatment at higher temperatures, the removal efficiencies for both stlicon and aluminum compounds are expected to be even higher.

Sulfur analyses of the solutions are being assembled and will be presented in a later report along with analyses of centrifuge solids and flaked caustic.

\section{PLANNED ACTIVITIES}

During the next quarter, the plant w111 be inspected for corrosion and erosion and mothballed for an estimated 9 month shutdown. Coal wt1l be packaged for shipment and processing wastes will be disposed. Data assessment will be continued and topical reports will be prepared for shakedown and operation. 